Portland State University

PDXScholar

1983

\title{
Liquid transmission line pulser circuit for laser excitation
}

Carl Stultz

Portland State University

Follow this and additional works at: https://pdxscholar.library.pdx.edu/open_access_etds

Part of the Applied Mechanics Commons

Let us know how access to this document benefits you.

\section{Recommended Citation}

Stultz, Carl, "Liquid transmission line pulser circuit for laser excitation" (1983). Dissertations and Theses. Paper 3345.

https://doi.org/10.15760/etd.5234

This Thesis is brought to you for free and open access. It has been accepted for inclusion in Dissertations and Theses by an authorized administrator of PDXScholar. Please contact us if we can make this document more accessible: pdxscholar@pdx.edu. 
AN ABSTRACT OF THE THESIS OF Carl Stultz for the Master of Science in Applied Science presented May, 1983.

Title: Liquid Transmission Line Pulser Circuit for Laser Excitation. APPROVED BY MEMBERS OF THE THESIS COMMITTEE
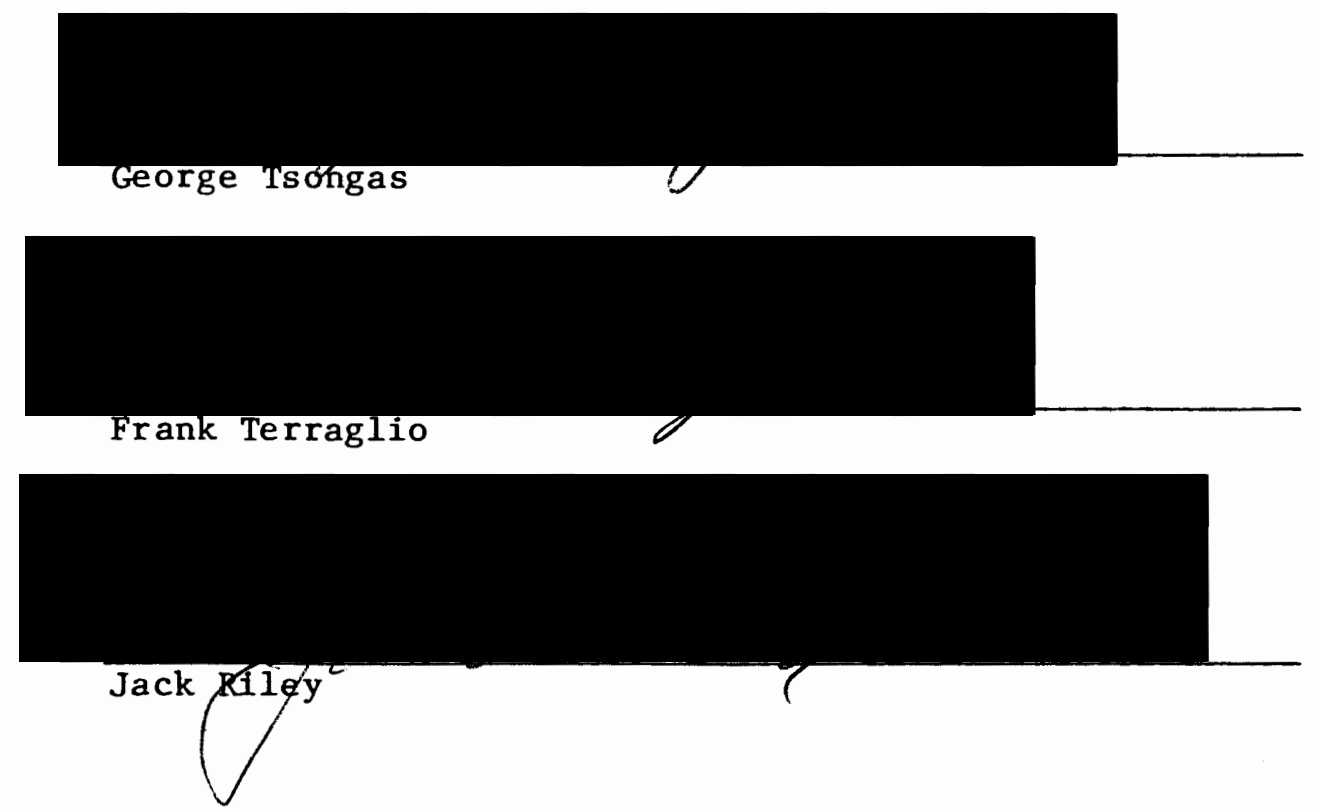

\section{ABSTRACT}

The subject of this thesis is the study of liquid dielectric transmission lines and their potential application as an excitation source in high speed, high-repetition rate laser designs. The design problems associated with the construction of high repetition rate (khz), short wavelength lasers have been reviewed and the major shortcomings of conventional electrical driving circuits identified. The identification of these shortcomings and a review of the available 1iterature on electrical circuits for various types of lasers suggested that liquid dielectrics and transmission lines could potentially have significant 
advantages over more conventional circuitry used in high repetition rate, short wavelength lasers. The construction of a set of three parallel electrode, liquid dielectric transmission lines was undertaken along with the associated electrical circuitry necessary for efficiently charging the system. The resulting electrical driving network was capable of delivering high voltage, $50 \mathrm{nsec}$ square wave current pulses at repetition rates exceeding $1 \mathrm{khz}$. The major problems limiting the practical usefulness of the system include the design tolerances required of the electrode separation structure and the requirements for a circulation system to deionize and decontaminate the liquid dielectric in designs requiring high reliability of the electrical driving circuitry. 


\title{
LIQUID TRANSMISSION LINE PULSER CIRCUIT
}

FOR LASER EXCITATION

by

CARL DEAN STULTZ

A thesis submitted in partial fulfillment of requirements for the degree of

\author{
MASTER OF SCIENCE \\ in \\ APPLIED SCIENCE
}

Portland State University

1983 
TO THE OFFICE OF GRADUATE STUDIES AND RESEARCH:

The members of the Committee approve the thesis of

Carl D. Stultz presented May 18, 1983.
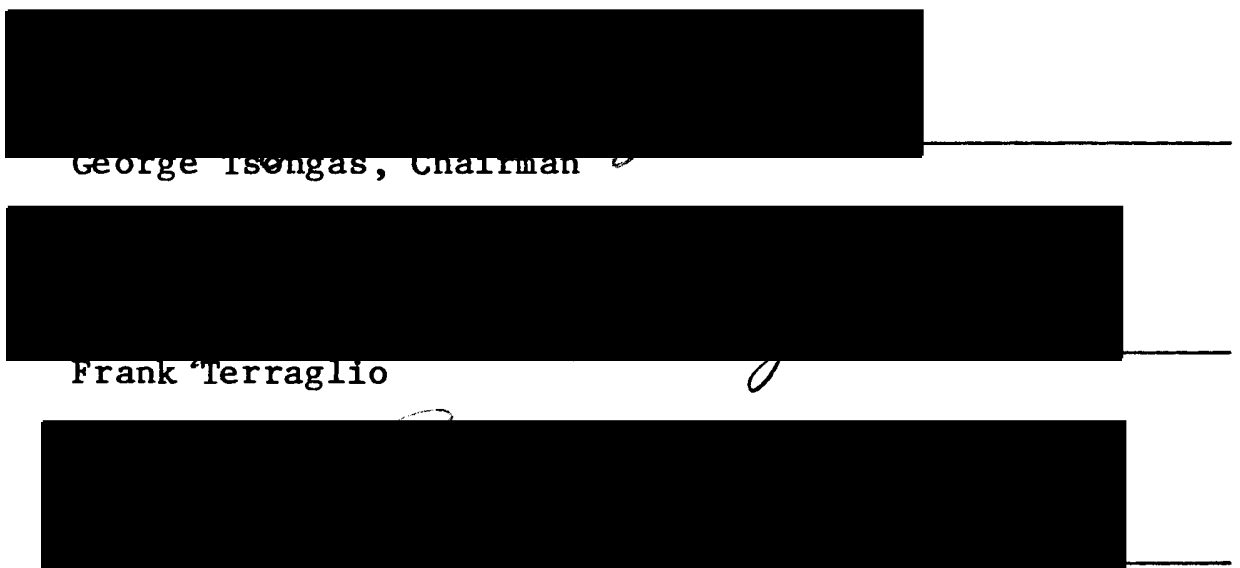

Jack Riley

APPROVED:

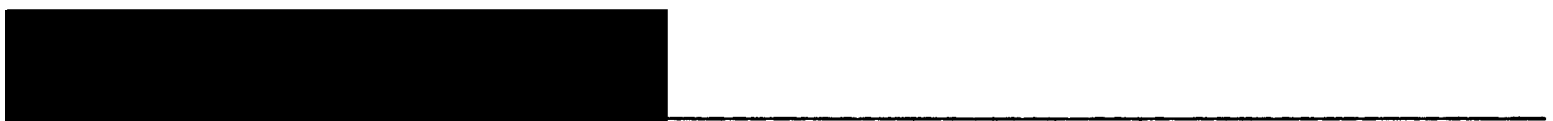

C. William Savery, Gad, Department of Mechanical Engineering

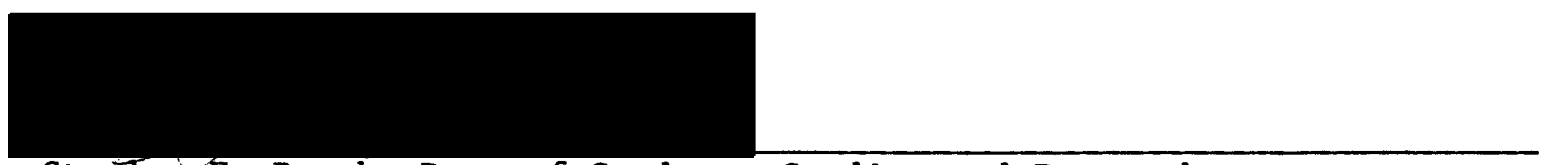

Stanley E. Rauch, Dean of Graduate Studies and Research 


\section{ACKNOWLEDGMENT}

The author wishes to thank the individuals and organizations who played such an important role in this research effort, especially George Tsongas, my major professor at PSU, advisor and friend. George Tsongas was not only the major impetus for the author's attending graduate school, but was instrumental in obtaining a graduate assistantship for the author and research funds for buying much needed supplies for the research effort. George is to be further commended for his unending patience with this graduate student in the completion of his thesis.

The author is also indebted to graduate student Joel Johnston for his review and helpful hints on the electrical driving apparatus studied in this research effort.

Additional thanks go to Dr. Thomas Cook for his helpful discussions and work and especially his comments on this thesis.

The author further wishes to thank the Physics Department's machine shop technicians whose excellent work and guidance made much of this research project a success.

The author is very grateful to the Office of Naval Research for the major portion of the funds used in this research project. Last, but not least, a thanks goes to Portland State University for providing the facilities and teachers who made attending PSU not only a good learning experience but a great pleasure as well. Moreover, it cannot be overemphasized the importance that attending graduate school has played in the author's life. The trials and 
tribulations of research work has greatly increased the author's confidence in himself and an awareness of the enormous potential of research itself. 


\section{PREFACE}

The research effort presented in this thesis originated out of the need identified by Los Alamos Scientific Laboratories and others for a reliable, long lived, high repetition rate electrical driving network for use in various short wavelength laser excitation schemes. This work was initiated in the fall of 1975 with an extensive literature search on the electrical circuits used in electric discharge and flashlamp pumped lasers.

Initial efforts in the design stage centered on improving flashlamps used to pump dye lasers. Later these efforts were expanded to include direct electrical discharge excitation schemes as well. Review of conventional circuitry used in these types of systems identified the energy-storage capacitor as the weak-1ink precluding long lived, high repretition operation. Typical lifetimes for the lowinductance, high energy density capacitors used in these circuits varied from a low of 10,000 to a high of several million discharge cycles. At $1 \mathrm{khz}$ repetition rates the lifetimes of these type capacitors are quickly extinguished.

A literature review of various types of gas, liquid and solid dielectrics use in capacitive energy storage schemes identified several liquids, notably glycerine, water and oil, whose electrical properties appeared favorable to the design of an electrical driving network with the desired characteristics. Liquid dielectrics were chosen due to their high energy-density storage capabilities for short duration pulses, and because they are self-healing in case an internal fault 
condition occurs. Transmission type pulse lines were used to provide square wave approximation pulse shapes for potential laser applications.

The actual transmission lines were constructed in the summer of 1976 with funds obtained on an internal grant from the PSU Research and Publications Committee. The experimental work with this system covered the period from late 1976 to early 1977. Due to the lack of a suitable power supply, repetition rates of only 10 to $20 \mathrm{hz}$ were attainable.

Work slowed in the summer of 1977 due to the author's employment outside the university and due to the fact he inherited a readymade family with whom he spent a considerable amount of time.

In the fall of 1977 a grant was obtained from the office of Naval Research to further study the liquid-dielectric transmission line concept. A high voltage power supply was purchased and enabled the author to refine the equipment and measurement capability and extend the operating range of the liquid-dielectric pulser circuit to as high as $2 \mathrm{khz}$ repetition rates.

Later work on the thesis itself was slowed by full-time employment of the author and a period of sabbatical leave by his advisor. The inordinate length of time spent on this work must in the last instance fall on the shoulders of the author for not more tenaciously setting goals and schedules for their completion. Nevertheless, the experience gained in this endeavor has taught the author how to better manage his time and research projects. 


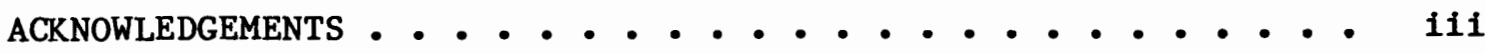

PREFACE. . . . . . . . . . . . . . . . . . . . v v

LIST OF TABLES . . . . . . . . . . . . . . . . . . ix

LIST OF FIGURES. . . . . . . . . . . . . . . . . . x

LIST OF SYMBOLS . . . . . . . . . . . . . . . . . xili

1. INTRODUCTION • . . . . . . . . . . . . . . . . . 1

Background . . . . . . . . . . . . . . . . 1

Scope of Study. . . . . . . . . . . . . . 2

2. REVIEW - . . . . . . . . . . . . . . . . . . 6

General Pumping Techniques . . . . . . . . . . 6

Hi-Intensity Spark Discharge Circuits . . . . . . . 8

Self-Terminating Laser Candidate Systems . . . . . . 11

3. FAST ELECTRICAL DISCHARGE TECHNIQUES . . . . . . . . . • . 15

Introduction. . . . . . . . . . . . . . 15

Energy Storage Elements . . . . . . . . . . 16

The Capacitor . . . . . . . . . . . 17

Liquid Dielectrics. . . . . . . . . . . . 22

Pulse Shape ................ . . 24

4. CHARGING CIRCUITRY . . . . . . . . . . . . . . . 29

Introduction. . . . . . . . . . . . . . 29

Resistive Charging. . . . . . . . . . . 30

Resonant Induction Charging . . . . . . . . . 34

5. HIGH POWER PULSER DESIGN . . . . . . . . . . . . . . 41

Transmission Line Geometry and Power Transfer . . . . . 41 
Pulse Charging Circuits. ............ 47

Charging Network . . . . . . . . . . 52

6. EXPERIMENTATION . . . . . . . . . . . . 58

Testing Overview . . . . . . . . . . . 58

Marx-Bank Test ............... 59

Water Tests. . . . . . . . . . 62

Glycerine Tests. . . . . . . . . . . 65

Self-Healing Times ............ 65

TL Charging Voltage Waveforms. . . . . . . . 66

Capacitive Matching. . . . . . . . . . . 73

Current Risetime Experiments . . . . . . . . 81

7. CONCLUSION . . . . . . . . . . . . . . 98

RECOMMENDATIONS. . . . . . . . . . . . . 102

APPENDIX 1. . . . . . . . . . . . . . . 106

APPENDIX 2 . . . . . . . . . . . . . . . 109

REFERENCES . . . . . . . . . . . . . . . 110 
LIST OF TABLES

Table

I Solid and Liquid Dielectrics Used in Pulse Power Technology . . . . . . . . . .
Page

23

II Tabulated Data and Results for the LTL Experiments . . . . . . . . . . . 83 
LIST OF FIGURES

Figure

$\underline{\text { Page }}$

3.1 Block diagram for a high speed discharge circuit . . . . 15

3.2 Pulse shapes of lumped parameter and distributed parameter networks . . . . . . . . . . . . 25

4.1a Resistively charged circuit. . . . . . . . . . 32

4.1b Capacitor voltage and current waveforms in a resistively charged circuit . . . . . . . . . . . 32

4.2a Inductively charged circuit. . . . . . . . . . 39

4.2b Capacitor voltage and current waveforms

in an inductively charged circuit. . . . . . . . . 39

4.3 Inductor-diode charged circuit . . . . . . . . . 39

4.4 Capacitor voltage and current waveforms

in the linear charging range . . . . . . . . . . 40

4.5 Capacitor voltage and current waveforms

in an inductor-diode charged circuit . . . . . . . . 40

5.1 Power transfer efficiency versus the
load/line ratio. . . . . . . . . . . . . 48

5.2 Liquid dielectric transmission line . . . . . . • 49

5.3 Marx-Bank and charging circuit schematic . . . . . . 53

5.4 Resonant charging circuit. . . . . . . . . . . 53

6.1 Hi-Voltage Marx-Bank . . . . . . . . . . 61

6.2 Marx-Bank erection spark gap . . . . . . . . . 61

6.3 Marx-Bank output voltage waveform. . . . . . . . 63

6.4 Marx-Bank output voltage wave form

6.5 Marx-Bank isolating spark gap. . . . . . . . . . 68

6.6 TL charging voltage waveform

(Anomalous breakdown). . . . . . . . . . . 68 
6.7 TL charging voltage waveform . . . . . . . . 69

6.8 TL charging voltage waveform . . . . . . . . 69

6.9 TL charging voltage waveform . . . . . . . . . 70

6.10 TL charging voltage waveform . . . . . . . . . . 70

6.11 New busbar configuration . . . . . . . . . . 74

6.12 Capacitor voltage, before and after breakdown. . . . . 74

6.13 Capacitor voltage, before and after breakdown. . . . . 76

6.14 Capacitor voltage. . . . . . . . . . . . 76 (143 pps)

6.15 Capacitor voltage. . . . . . . . . . . 78 (555 pps)

6.16 TL current output waveform . . . . . . . . . . 78 ( source capacitance $=6 \mathrm{TL}$ capacitance)

6.17 TL current output waveform . . . . . . . . . .

( source capacitance $=10 \mathrm{TL}$ capacitance)

6.18 TL current and voltage waveforms . . . . . . . . . 79 ( source capacitance $=$ TL capacitance)

6.19 TL current and voltage waveform. . . . . . . . . . 80 (source capacitance > TL capacitance)

6.20 Stainless steel pin electrode configuration. . . . . . 80

6.21 Low inductance output configuration. . . . . . . . . 84

6.22 TL current output waveform . . . . . . . . . . 84

6.23 TL current output waveform . . . . . . . . . . 85

6.24 TL current output waveform . . . . . . . . . 85

6.25 TL current output waveform . . . . . . . . . . 87

6.26 Spark electrode spacing vs. dI/dt. . . . . . . . . 87

6.27 Spark electrode spacing vs. load impedance . . . . . . . 90 
6.28 TL current output waveform . . . . . . . . . . 90

$$
\left(\mathrm{Z}_{\mathrm{TL}}=.4 \mathrm{ohm}\right)
$$

6.29 TL current output waveform . . . . . . . . . . 91

$$
\left(\mathrm{Z}_{\mathrm{TL}}=.4 \mathrm{ohm}\right)
$$

6.30 Short busing strip output configuration. . . . . . . 91

6.31 TL current output waveform . . . . . . . . . . 93

$$
\left(\mathrm{z}_{\mathrm{TL}}=3.6 \mathrm{ohm}\right)
$$

6.32 TL current output waveform . . . . . . . . . . 93

$$
\left(\mathrm{z}_{\mathrm{TL}}=3.6 \mathrm{ohm}\right)
$$

6.33 TL current and voltage waveforms . . . . . . . . 94

$$
\left(\mathrm{Z}_{\mathrm{TL}}=8.9 \mathrm{ohm}\right)
$$

6.34 TL current and voltage waveforms . . . . . . . . . 94

$$
\left(\mathrm{Z}_{\mathrm{TL}}=8.9 \mathrm{ohm}\right)
$$

6.35 Output current risetimes vs. TL impedance. . . . . . . 97 


\section{LIST OF SYMBOLS}

\begin{tabular}{|c|c|}
\hline A: & Electrode Area \\
\hline$\infty:$ & Proportionality Constant \\
\hline$c:$ & Speed of Light in a Vacuum \\
\hline C: & Capacitance \\
\hline $\mathrm{C}_{\mathrm{s}}:$ & Source Capacitance \\
\hline $\mathrm{C}_{\mathrm{TL}}:$ & Transmission Line Capacitance \\
\hline$S:$ & Dielectric Loss Coefficient \\
\hline$\in:$ & Dielectric Permittivity \\
\hline$\epsilon_{0}:$ & Permittivity of Free Space \\
\hline f: & Repetition Date \\
\hline$f_{o}:$ & Resonant Frequency \\
\hline I: & Current \\
\hline $\mathrm{I}_{\mathrm{o}}:$ & Output or Load Current \\
\hline $\mathrm{J}:$ & Joules \\
\hline $\mathrm{K}:$ & Dielectric Constant \\
\hline$\ell:$ & Electrode Length \\
\hline L: & Inductance \\
\hline $\mathrm{L}_{0}:$ & Output Inductance \\
\hline$t:$ & Time Variable \\
\hline $\mathrm{T}:$ & $\begin{array}{l}\text { Time Period or Pulse Duration } \\
\text { (i.e., Pulse Length) }\end{array}$ \\
\hline $\mathrm{T}_{\mathrm{c}}:$ & $\begin{array}{l}\text { Pulse length for a critically damped } \\
\text { capacitive discharge }\end{array}$ \\
\hline $\mathrm{T}_{\mathrm{R}}:$ & Resonant Charging Time \\
\hline
\end{tabular}




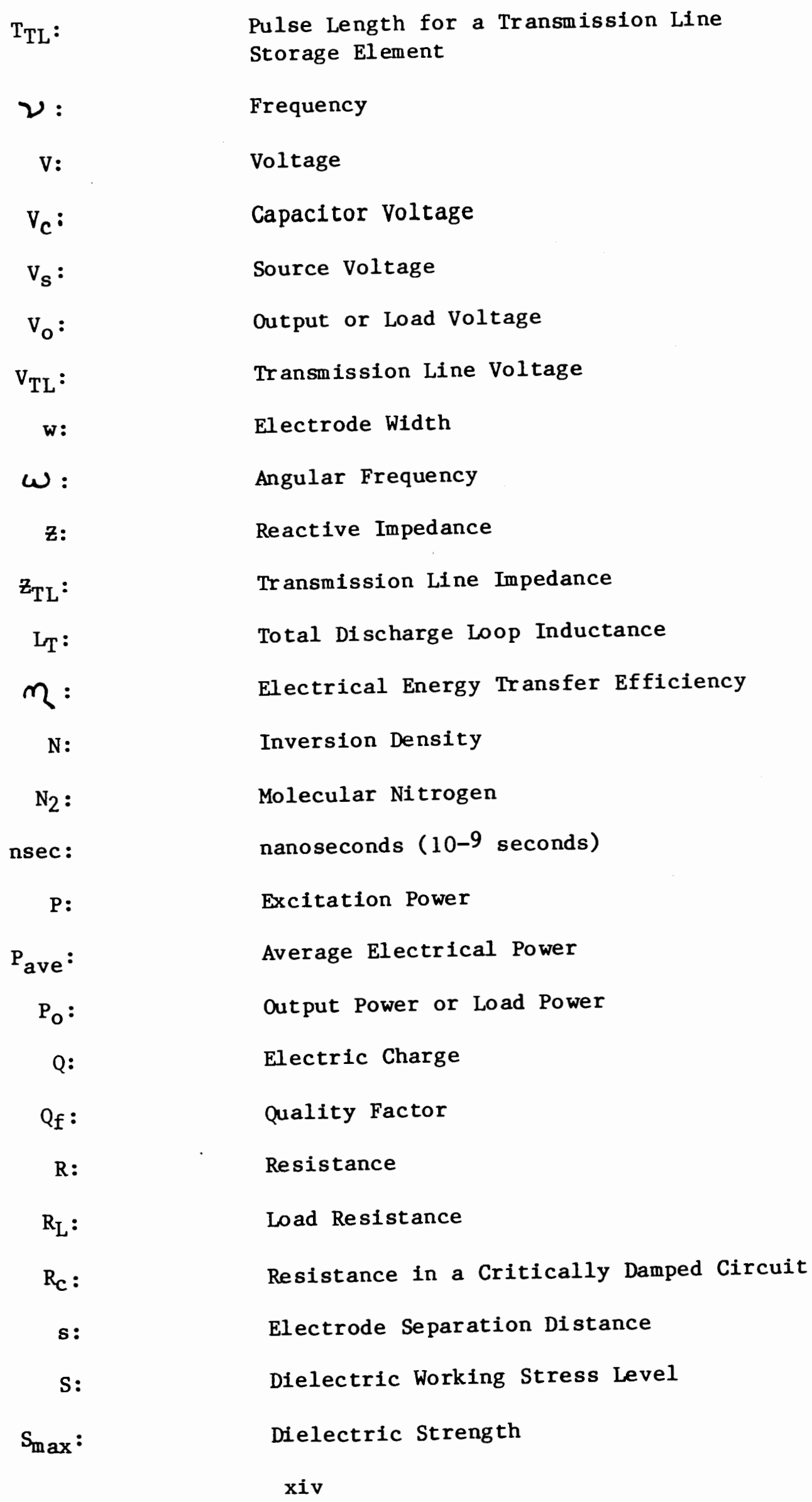

S:

$\mathrm{S}_{\max }:$

Capacitor Voltage

Source Voltage

Output or Load Voltage

Transmission Line Voltage

Electrode Width

Angular Frequency

Reactive Impedance

Transmission Line Impedance

Total Discharge Loop Inductance

Electrical Energy Transfer Efficiency

Inversion Density

Molecular Nitrogen

nanoseconds (10-9 seconds)

Excitation Power

Average Electrical Power

Output Power or Load Power

Electric Charge

Quality Factor

Resistance

Load Resistance

Resistance in a Critically Damped Circuit Electrode Separation Distance

Dielectric Working Stress Leve1

Dielectric Strength

xiv 


\section{INTRODUCTION}

Background

In recent years there has evolved considerable interest in the development of lasers operating in the ultra-violet region of the spectrum. It is here that many of the electronic transitions in atoms and molecules occur(1) and a tunable, narrow band source of coherent radiation would greatly facilitate the study of the electronic structure of atoms and molecules. Ultraviolet sources are also urgently needed for a wide variety of photochemical processes, most notably that of isotope separation.(2) These two applications alone have warranted the growth of extensive research programs throughout the world to develop UV (ultra-violet) lasers of various types. The present lack of suitable lasers in this region as compared to the proliferation of sources that has appeared in the visible and infrared portions of the spectrum can best be understood by a review of the fundamental considerations in obtaining laser action. The basic difficulty in obtaining lasing in this region of the spectrum was first noted by Schalow and Townes(3) in their original analysis on infrared and optical lasers. They showed that the required excitation power needed to establish useful inversion densities was strongly frequency dependent. The results of their analysis shows that the dependence of inversion density $\mathrm{N}$ on excitation power $\mathrm{P}$ and lasing frequency is

$$
\Delta \mathrm{N} \propto \mathrm{P} \quad \mathcal{\nu}-4
$$

This clearly shows the difficulties encountered in achieving useful inversion densities and laser action at short wavelengths or high frequencies. 
Scope of Study

It is the partial intention of this study to review the present* methods for attaining the high levels of power needed to successfully pump a UV 1aser. It has been found in a review of the literature that only too often lasers have been designed with respect to the convenience of available components and simplicity of construction. This design procedure is entirely justifiable in many instances, but applications such as isotope separation require an especially efficient use of the available power. In particular, a unique electrical driving network has been designed, constructed and tested to verify that it could not only deliver the necessary input powers for obtaining UV laser action, but show needed improvement in performance with respect to reliable, high power capabilities. These characteristics are of special interest to laser systems destined for isotope separation and other wavelength selective chemical synthesis techniques because of their possible application to commercial processes.

A review of the literature on the electrical driving networks used in many short wavelength laser schemes showed that the weakest link precluding reliable, high repetition rate operation was the energy storage capacitor used in the high speed discharge circuits. The high speed requirements were met by using very high voltage, low inductance capacitors. The low inductance in these type of capacitors was partially achieved by minimizing the positive-negative electrode

*See Preface 
separation distance which also maximizes the dielectric stress. Maximizing the dielectric stresses of a capacitor almost invariably leads to short capacitor lifetimes because sooner or later an internal fault or breakdown w11 occur in the dielectric itself.

It was for this reason that another literature search was made on various types of dielectrics including gases, liquids and solids in order to find one with electrical characteristics more favorable to the design of a long lived, low inductance capacitive storage system. The literature search revealed that several liquids, most notably transformer oil, water and glycerin had many of the desired electrical characteristics. A electrical circuit was then designed to take advantage of the properties of liquid dielectrics. Thus, the design of the driving network used in the present research effort centers around the use of liquid filled transmission lines which generate rectangular shaped output pulses when discharged into matched resistive loads. Liquid dielectrics were used in preference to solids because of their self-healing characteristics under fault conditions. of all the liquids considered, glycerine appeared to be the best choice because of its high dielectric constant $(k=44)$, good breakdown strength $(>20 \mathrm{kv} / \mathrm{mm})$, and electrical stability under no flow conditions. Pure water was initially selected for use, but subsequent experimentation revealed that ionization products degraded the electrical properties so fast that only continuous deionization would render it usable in the prototype system. Because the selected dielectric also suffered significant losses when left charged for more 
than a few usecs due to internal bleedoff, it was necessary to pulse charge it to make effective use of the available power. In this context two different types of pulse charging networks were constructed and used in the various experimentation phases of the research project. The first consisted of a low inductance, two stage Marx bank resistively charged by a $17 \mathrm{kv}, 100$ watt power supply. The second pulse charging network was based on a voltage doubling, resonant charging scheme utilizing a capacitor discharge. This second network was constructed in order to increase the power transfer efficiencies from the electrical mains to the transmission line storage elements. The latter circuit realized transfer efficiencies approximately 10 times greater than those obtainable with the Marx bank.

Initially, testing of the glycerine filled transmission lines was aimed at determining the practical attainable working stresses. Later experiments explored fault conditions, healing times and charging voltage waveforms. After the proper range of working conditions were determined, the experimentation was directed toward measurements of the current and voltage output waveforms under varying conditions of load impedence and inductance. Later work included extending the repetition rates from single shot experiments to high repetition rates in the low khz range.

During the course of the experimental work, a continuing study of potential laser applications was being made. This study, which was mainly an extensive literature search and review, revolved around trying to categorize that group of applications including 
laser mediums which might benefit from the characteristics of this particular type of electrical driving network. A list of these potential applications including further work that should be performed with liquid dielectric energy storage systems is discussed in the recommendation section of this thesis. In addition, because it was recognized that the next major step should involve incorporation of the excitation system into an actual laser, that was an expected follow-on project. Simultaneous analytical modeling of the transmission line was also anticipated. 


\section{REVIEW}

General Pumping Techniques

Two of the more prominent pumping schemes used today are that of electrical and optical pumping. The former is the most efficient theoretically because the latter introduces an intermediate conversion step (electrical to optical) which can only decrease total conversion efficiencies. Since both of the pumping techniques impact a wide variety of ultraviolet lasing mediums, both will be considered briefly with respect to their general operating characteristics. It is not the purpose of this investigation to address these techniques in their totality but to focus on the process of taking electrical energy from the utilities distribution system and delivering it to an appropriate load, whether it be a flashlamp or laser gas. Therefore, flashlamps and lasing mediums will be addressed only with respect to their effects on the driving network used and detailed laser and gas discharge kinetics will be avoided.

Optical pumping is presently the only practical method for attaining laser action in the various liquid and solid laser mediums discovered to date. The most well known liquid laser is the dye laser whose wide tuning capability has elevated it to a prominent place in many research laboratories. There are a number of dyes which operate in the ultraviolet with the lower end extending down to $340 \mathrm{~nm}$ in paraterphenyl. (4) Solid state lasers on the other hand do not presently operate directly in the ultraviolet, but must rely on nonlinear upconversion techniques for obtaining coherent UV radiation. 
Since this method involves not only electrical to optical to lasing conversion steps, but the additional one of a nonlinear nature it will be addressed no further because of its inherent overall inefficiency and inability to be scaled to high average powers. (5)

One of the most widely used pulsed light sources is that produced by a spark discharge in a high pressure gas.(6) The radiation is caused by the retardation of electrons in the field of positive ions (free-free transitions), electron-ion recombinations (free-bound transitions), and finally the radiation broadened lines of bound electron transitions (bound-bound transitions). To produce these high brightness light sources it is common to use a high voltage, low inductance discharge circuit and a high pressure flashlamp. It should be pointed out that high voltage, low inductance discharge circuits are also used in direct electrical pumping schemes as we1l. This common link is very important to the latter systems because of the wealth of information available on high intensity light sources. Long before the advent of the laser, considerable amounts of data were amassed concerning high power spark discharges. Much of this information is related to the complex interactions between driving source and gas load. Detailed studies have also been made of the spectral output of various types of discharges. This latter information is an excellent indicator of energy level populations and by correlating the spectral output with the circuit parameters used in these studies it is possible to size relevant circuit parameters when designing various types of gas lasers, UV or otherwise. It is because of this common 
connection that spark discharges are so important to the development of efficient gas lasers. It is also the reason why many of the design criterion used in this investigation on fast discharge pumping schemes were derived from spark discharge studies.

\section{High Intensity Spark Discharge Circuits}

High brightness, ultraviolet rich spark discharges are generally produced from high voltage, low inductance discharge circuits. The high voltage, low inductance requirements insure the maximum possible speed of energy input from the capacitor to the discharge channel. An idea of how these parameters actually affect the power input can be gained by analyzing the current-time dependency of a damped RLC oscillation. It is well known that the RLC discharge current is given by the expression(7)

$$
I(t)=\frac{V_{C e^{-(R t / 2 L)}}}{\left[\frac{\mathrm{L}}{\mathrm{C}}-\frac{\mathrm{R}^{2}}{4}\right]^{1 / 2}} \sin \left[\frac{1}{\mathrm{LC}}-\frac{\mathrm{R}^{2}}{4 \mathrm{~L}^{2}}\right]^{1 / 2} t
$$

For the case of an underdamped oscillation (i.e. $1 / \mathrm{LC} \gg \mathrm{R}^{2} / 4 \mathrm{~L}^{2}$ ) expression (2) reduces at the beginning of the discharge to

$$
I(t)=\frac{V_{C}}{(L / C)} 1 / 2 \operatorname{sIN}(1 / L C)^{1 / 2} t
$$

where Vo is the initial capacitor voltage, $C$ the capacitance and $\mathrm{L}$ the total loop inductance of the discharge circuit. Inspection of 
equation (3) will show that $(\mathrm{L} / \mathrm{C})^{1 / 2}$ has the units of ohms and is usually termed the reactive impedance ( $z$ ) of the discharge circuit. $(1 / L C)^{1 / 2}$ on the other hand has the units of frequency and represents the angular oscillation frequency (w) of the discharge. The rate of power input to a resistive load is to a first approximation given by the time rate of current buildup $d I / d t$ in the channel. Differentiation of (3) shows that initially this time rate of change $d I / d t$ depends only on the voltage and inductance of the discharge circuit.

$$
(d I / d t)_{t=0}=V_{c} / L
$$

The maximum value of the current and hence power input is given in an underdamped circuit by

$$
I_{\max }=V_{c} / z
$$

where $\mathrm{Z}$ represents the reactive impedance $(\mathrm{L} / \mathrm{C})^{1 / 2}$ of the discharge circuit.

Based on the above considerations the rate of power input to a high pressure spark discharge can be increased by raising the voltage, lowering the circuit inductance or both. In order to construct high intensity spark light sources it becomes necessary to use high voltage, low inductance capacitors coupled with the proper busing network for connecting the capacitors to the discharge gap. To reduce the inductance of the discharge circuit to a minimum possible value the arrangement of the circuit elements should be such that the magnetic field formed by the current is contained in the smallest possible 
volume. It should be noted that with a given set of circuit components the maximum power input occurs under critically damped conditions. That is all of the energy stored in the capacitor is delivered to the spark load in approximately the first half period of oscillation instead of being spread out over many periods as in the underdamped case. The energy stored in a capacitor is

$$
E=1 / 2 \mathrm{CV}_{\mathrm{c}}{ }^{2}
$$

where $V$ is the charging voltage and $C$ the capacitance. The first half period is given by

$$
T=\pi(L C) 1 / 2
$$

with $\mathrm{L}$ being the total discharge circuit inductance. The average power input during the discharge pulse is then

$$
P_{\text {ave }}=v_{c}{ }^{2 / 2 \pi} z
$$

where $\mathrm{Z}$ is again just the reactive part of the total discharge impedance. The total discharge impedance is the sum of the reactive and resistive parts. It happens that for the critically damped case the reactive component is one-half the resistive part. (8)

$$
R_{C}=2(L / C)^{1 / 2}
$$

Therefore, the average power could alternatively be given as

$$
P_{\text {ave }}=V^{2} / \pi R_{c}
$$


It should be clarified that these results are only first approximations because the actual energy delivery during a critically damped pulse extends somewhat beyond the first-half period. Even so this analysis does show the relative effect that various circuit parameters have on the power input to a spark discharge. It should also be realized that because of the changing discharge impedance with respect to time during breakdown, exact matching between the reactive and resistive parts of the circuit is not entirely possible. It is known that the spark impedance during breakdown goes from a very high value to a small fraction of an ohm.(9) Because of this changing impedance, discharge circuits are usually designed to deliver maximum power after the discharge has leveled off to its low impedance level. A critically damped discharge pulse is also, because of its limited length, the most efficient pulse shape for transferring electrical energy from a source to a coherent optical output. This second characteristic is the result of temporal changes in the populations of various energy levels of the lasing medium of interest.*

\section{Self-Terminating Laser Candidate Systems}

The following is a discussion of three particular types of lasing systems which benefit from using a critically damped discharge pulse (i.e., one of a limited length) in the excitation scheme used to achieve lasing conditions. These three systems all lase at short wavelengths and are potential candidates for use in high repetition rate, chemical process oriented applications.

*This may also apply to mediums not directly involved in the lasing process such as dye solvents or host mediums in solid state lasers. 
There are many types of lasers whose outputs terminate prematurely before the conclusion of the pumping pulse. The molecular nitrogen laser operating at $3371 \&$ in the ultraviolet is one of the most well known of these self-terminating lasers. The reason for this self-termination in the nitrogen laser is due to the fact that the lower laser level $\left(B^{3} \pi g\right)$ has a lifetime of about 6 usecs, more than two magnitudes greater than the 40 nsec lifetime of the upper level ( $\left.c^{3} \pi g\right) .(10)$ Population inversion can be achieved because the excitation cross section of the upper level is about twice as large as the lower one, so using a very fast discharge the system can be inverted. The long lifetime of the lower level as compared to the upper is the reason for the self-terminating nature of this laser. The lower level bottlenecks the electronic flow, effectively destroying the inversion condition and lasing. It is for this reason that the discharge pulse lengths necessary to efficiently pump the $\mathrm{N}_{2}$ laser should be no longer than the upper state lifetime or about $40 \mathrm{nsec}$. For a discharge pulse longer than this much of the energy will be needlessly wasted.

The flashlamp pumped dye laser also has a type of selfterminating characteristic associated with it. The operating principle of a dye laser is much the same as for other lasers. The dye molecules are raised to the lowest singlet state directly or more often by relaxation from some higher lying singlet state. Lasing involves a return to a lower level by stimulated emission. These processes are further complicated by nonradiative transitions to the lower level, singletstate absorption of the lasing wavelength and more importantly, 
intersystem crossing from the upper singlet level to a long-lived triplet state which can also absorb at the lasing wavelength.(11) These processes are not as detrimental to lasing as in the $\mathrm{N}_{2}$ bottlenecking case, but intersystem crossing and subsequent absorption at the lasing wavelength severely restricts the levels of wall plug conversion efficiences obtainable for times longer than that required for intersystem crossing to occur ( $100 \mathrm{nsec}$ or more). Even though there exists quenching agents that do prevent a large accumulation of molecules in the triplet state, (12) any molecules that do cross over represent a loss in efficiency. Therefore, here too, the maximum overall wall plug conversion efficiencies should be attained with short high power pump pulses.

The last system to be discussed concerning self-termination and probably the most promising today regarding the development of a high power, efficient, ultraviolet laser is the rare gas mono-halide group. This group of lasers is based on gaseous excimers. An excimer is a molecule which only exists in the excited state and dissociates when it reaches the ground state. This is because the ground state is weakly bound or even repulsive and the only stable state is the excited one. Since the terminal state is very unstable it exists only for about $10^{-13}$ second (i.e. that time required for molecular vibration). This extremely fast depopulation prevents the terminal state bottlenecking problem of the nitrogen laser. The fact that the ground state is also the lower laser level is one of the reasons for the high quantum efficiencies of this laser. There are other factors which 
contribute to this laser group's high overall efficiency, but what we are concerned with here is the the self-termination characteristic that also shows up in the rare-gas mono halides.(13) All of the reasons are as yet unclear, but present evidence seems to indicate that both gas clean-up and discharge runaway (arcing) are major causes for premature laser pulse termination. Whatever the reason it appears that efficient operation of UV wavelength rare gas halides may also require short pulse length electrical discharges.

These previous three cases of self-terminating laser action have been used to illustrate the fact that the efficient transfer of energy from a source to a coherent optical output may not only require a high rate of power input in accordance with equation (1), but should also satisfy a temporal requirement as we1l. The most efficient pulse shape or duration for exciting many types of UV lasers has been found to be of a very limited length usually much less than a usec. This additional requirement of short pulse length poses a very serious reliability problem with respect to the components that make up the discharge circuit. This reliability problem arises because a short pulse length is achieved by using very low inductance circuit elements including the primary energy storage element, the capacitor. As mentioned earlier, low inductance capacitors have very limited lifetimes in high voltage, fast discharge (i.e., short pulse length) applications. See the section on capacitors for a more detailed explanation of this problem. 
Introduction

The discharge circuits used to directly pump various UV laser mediums must at least satisfy equation (1) and should have a short pulse length for maximizing transfer efflciencies. The actual pulse length will be determined primarily by the lasing medium selected because of the need for the pulse excltation length or time to be less than that required for self-termination to occur in the selected lasing system.

Figure 3.1 shows a schematic dlagram of the usual arrangement of electrical components in a fast, high power discharge circuit.

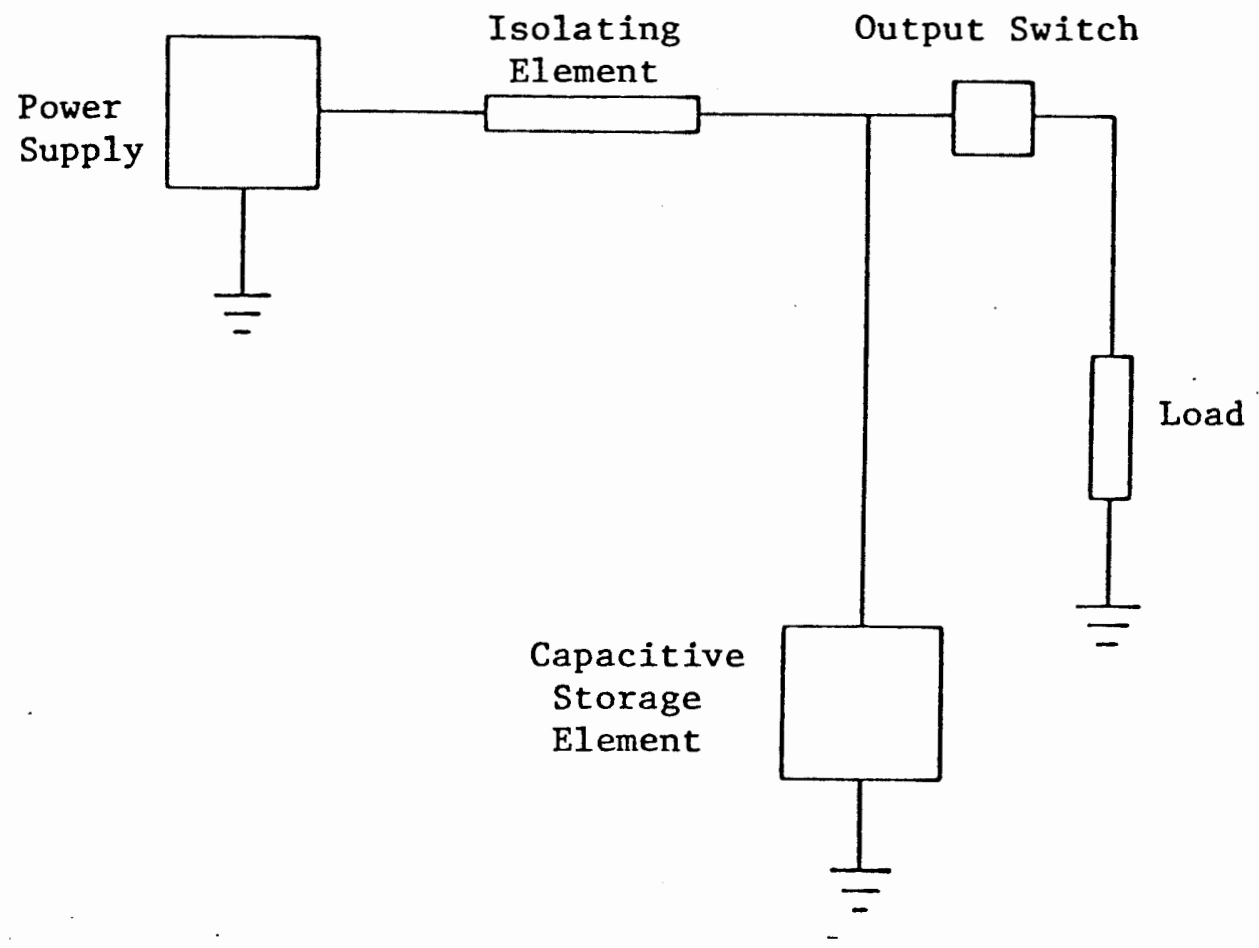

Fig 3.1. Block diagram for a high speed discharge circult 
The discharge circuit proper usually consists of an energy storage element, an output switch and of course an appropriate load, be it a flashlamp or laser gas medium. In reality any practical high power electrical driving circuit not only includes a discharge circuit but also a circuit to charge the energy storage element as shown in Figure 3.1. Notice that the storage element is common to both the charging and discharging sections of this circuit. There are many different varieties of these two circuits, each with its advantages and disadvantages. The following sections are a review of the various methods and components used in these types of circuits.

\section{Energy Storage Elements}

The two most general ways of storing and subsequently retrieving electrical energy are the capacitor which stores energy in an electric field and the inductor which utilizes a magnetic field for storage purposes. The latter form of storage has not yet been developed to the extent that capacitive storage has because of the lack of a suitable switch. For high energy systems (kilojoules) capacitive energy storage costs between $\$ .10$ and $\$ 1.00$ per joule depending on the complexity of the switching and energy transmission systems. The economics of an inductive system on the other hand is estimated to be at least an order of magnitude lower. Moreover, including the switch, an inductive system will be far more compact than a comparable capacitive system. Therefore, inductor storage switch development is underway at various laboratories around the world, but presently the capacitor is far easier to energize and switch across a load than an 
inductor. It is not then unexpected that present high power electrical discharge systems are designed around the capacitor with all its advantages and unfortunately, faults.

\section{The Capacitor}

The capacitor is one of the oldest forms of electrical energy storage devices for pulsed power requirements. Capacitance exists between any two electrical conductors. Basically a capacitor consists of two parallel conductors separated by a dielectric material. The actual energy storage occurs in the dielectric, and thus it plays a major role in the operation of a capacitor. When a direct voltage is applied to a capacitor, the electric field is distorted and the bound electric charges are polarized or displaced from their normal position of equilibrium. If the voltage per unit length (i.e. electric field strength) is increased beyond a certain point, the dielectric may break down, resulting in a short and failure in the capacitor. This factor is important in determining the applicability of any one dielectric towards capacitor construction and is characterized by the measurable quantity of dielectric strength which can be given in $\mathrm{kv} / \mathrm{mm}$. The total energy stored in a capacitor $(\mathrm{V} / \mathrm{s})$ is given by equation (6) to be

$$
\mathrm{E}=1 / 2 \mathrm{CV}_{\mathrm{c}}^{2}
$$

For a parallel plate capacitor the capacitance is given by reference (8) to be

$$
C=\epsilon A / s
$$


With $\in$ being the permittivity of the dielectric, $A$ the plate area and s the plate separation or dielectric thickness. Therefore, for a given $\epsilon, A$, and $\mathbf{s}$ which determine the capacitance of an electrical element, if the dielectric strength of the material used in the element is increased, then the applied voltage that can be used on this element can also be increased. Referring back to equation (6), an increase in the usable voltage will lead to an increased energy storage capability for a given size capacitive element. This fact is more properly represented by an increase in energy density, which is the energy stored per unit volume of dielectric material. The only other parameter that can increase the energy density is the dielectric permittivity. It is the permittivity and the dielectric strength which affect the construction of high energy density capacitors. The delivery ability of any capacitor with respect to speed is reflected by the system's inherent inductance. Low inductance is required for fast energy delivery because during discharge of a capacitor the impulse current branches off energy to the magnetic field equal to $1 / 2 \mathrm{LI}^{2}$ (see reference 9) which reduces the first peak value of the impulse current; $L$ is the inductance of the discharge loop and I the impulse loop current. The energy absorbed by the magnetic field is not lost but becomes effective during the latter part of the discharge. With proper circuit design most of the inductance found in the discharge circuit will be the self-inductance of the capacitor itself.

The optimum pulse shape for maximizing transfer efficiencies has been shown to be a critically damped one. The pulse width for the 
critically damped case is given by equation (7), as follows:

$$
T=\pi(L C)^{1 / 2}
$$

This expression shows that critically damped discharge pulse lengths are proportional to the square root of the inductance-capacitance product. The average power delivered during the time of this pulse is given by: ( 8$)$

$$
P_{\text {ave }}=v_{c}^{2} / 2 \pi z
$$

Inspection of these two equations will show that for a given voltage and capacitance the pulse length can be reduced and the power increased by lowering the total inductance in the discharge loop. The difficulty in practically accomplishing this is the strong interdependence between the inductance and capacitance of an energy storage capacitor. The inductance of a simple parallel plate is equal to:(14)

$$
L=\mu s l / w
$$

where $\mu$ is the permeability of the dielectric, $s$ the plate separation, 1 the plate length and $w$ the plate width. Using equations (11) and (12), we can rewrite the expressions for the pulse length and average power .

$$
\begin{gathered}
T=\pi(\mu \epsilon) 1 / 2 \\
P_{\text {ave }}=\left(v^{2} w / 2 \pi s\right)(\epsilon / \mu) 1 / 2
\end{gathered}
$$

Equation (14) shows the desired result for discharge pulse lengths. 
For a given dielectric,fand $\mathcal{H}$ will be constant and the length of the plates will determine the pulse length. Likewise for a set of plates of a given length, the pulse length can be changed by using dielectrics with varying permittivities (i.e. dielectric constant). Equation (15) is slightly more complex and requires an additional modification before certain characteristics can be highlighted. It has been shown previously that the energy density of a capacitor is directly proportional to both the dielectric strength and the permittivity. The dielectric stress is equal to $\mathrm{V} / \mathrm{s}$ and this factor appears in (15) as well as the square root of the permittivity. Rewriting equation (15) we introduce the dielectric working stress level $\mathrm{S}$ which is equal to $\mathrm{V} / \mathrm{s}$. The dielectric strength is just $S_{\max }$ which is the maximum stress that be applied to a dielectric above which dielectric breakdown will occur.

$$
P_{\text {ave }}=(V S w / 2 \pi)(\epsilon / \mu)^{1 / 2}
$$

From electromagnetic field theory we can obtain identities for the various forms that the combinations of the permittivity and permeability have taken. The term $(\epsilon / \mu)^{1 / 2}$ is the inverse of the speed of light in the dielectric and equals $(K)^{1 / 2} c$ where $K$ is the dielectric constant of the medium, whereas $(\mathrm{C} / \mathrm{L})^{1 / 2}$ is the reciprocal of the impedance of the dielectric. The ratio of the voltage $V$ to the impedance $\mathrm{Z}$ is by Ohm's Law the short-circuit current $\mathrm{I}$. Rearranging equation (14) and (15)

$$
\begin{aligned}
& T=(T / c)(k)^{1 / 2 l} \\
& P_{\text {ave }}=I S w / 2
\end{aligned}
$$

*The impedance of a capacitor is equal to $(\mathrm{L} / \mathrm{C})^{1 / 2}$. 
The last expression shows that for a simple strip line geometry the average power output of the device can be raised by increasing the short-circuit current, the width of the lines or by maximizing the dielectric stress. It should be realized that all these parameters are not independent of each other. For a given dielectric and strip line geometry and hence impedance, the maximum current occurs when the dielectric is stressed to its maximum value without failing (i.e., Io $\rightarrow I_{\max }$ when $\left.S \rightarrow S_{\max }\right)$. If one stresses a dielectric to such high levels in order to maximize the current it severely limits the lifetime of the dielectric under repetitively pulsed conditions. It has been found empirically that most high energy density capacitors follow the we11 known "fifth power law."(15) This empirical derived law shows that the lifetime $\left(\mathrm{T}_{\mathrm{L}}\right)$ of these types of capacitors are inversely proportional to the fifth power of the dielectric stress.

$$
\mathrm{T}_{\mathrm{L}} \propto \mathrm{S}^{-5}
$$

As an example of the effect that these relationships have on the lifetime of a capacitor, assume that two identical capacitors are charged up. The first capacitor is charged to the maximum voltage corresponding to $\mathrm{S}_{\max }$ - Following equation (18), its lifetime will be inversely proportional to the fifth power of $S_{\max }$. If the second capacitor is charged up to only one-half the voltage of the first, its lifetime will be inversely proportional to the fifth power of $1 / 2$ $S_{\max }$ A simple calculation shows that the second capacitor will have a lifetime of 32 (i.e., 2 raised to the fifth power) times that of the first. 
Some of the more promising dielectrics developed for use in high energy density capacitors such as the polyester films have shown an even poorer dependency of dielectric lifetimes to dielectric stress levels when stressed to high levels of electric fields.(16) For the mylar polyester films

$$
\mathrm{T}_{\mathrm{L}} \propto \mathrm{s}^{-7}
$$

This means that for a given stress level polyester dielectrics have much shorter lifetimes than those types whose lifetimes follow equation (18).

Present high energy density capacitors are limited to energy densities of about 50 joules/1b with dielectric strengths not exceeding $150 \mathrm{kv} / \mathrm{mm}$. (19) Commercially available, low inductance units usually have lifetimes of not much more than 100,000 shots.(17) At a repetition rate of 100 Hertz the useful lifetime would be used up in less than 2 minutes, necessitating complete replacement of the burned out capacitor.

This problem of limited lifetime in high density, low inductance capacitors has been a stumbling block in the development of reliable and compact short pulse length, high power, high repetition rate electrical driving networks for UV laser excitation.

\section{Liquid Dielectrics}

One possible solution to the limited lifetimes in highly stressed solid dielectrics is found in the use of self-healing liquid dielectrics such as water, glycerine and transformer oils. All three 
of these liquids have been used with great success in large energy, high power electron beam machines. One of the primary reasons liquids are used in such devices is that costly downtime and repairs are not necessary if a short circuit and fallure does occur in the dielectric. Water also has the additional advantage of being readily available, inexpensive and easily cleaned up if spilled. Table 1 lists some of the more commonly used dielectrics in high power systems and some of their more important electrical properties.(18)

Table 1. Solid and Liquid Dielectrics Used In Pulse Power Technology(18)

\begin{tabular}{|c|c|c|c|c|}
\hline Dielectric & $\begin{array}{l}\text { Dielectric } \\
\text { Constant K }\end{array}$ & $\begin{array}{l}\text { Pulse Length Per } \\
\text { (cm) of Dielectric } \\
\text { (NSEC/cm) }\end{array}$ & $\begin{array}{c}\text { Effective Dielectric } \\
\text { Strength } \\
(\mathrm{KV} / \mathrm{cm})\end{array}$ & $\begin{array}{c}\text { Energy Storage } \\
\text { Capability } \\
\left(\mathrm{J} / \mathrm{cm}^{3}\right)\end{array}$ \\
\hline Water & 80 & .60 & 15 & .12 \\
\hline Glycerine & 40 & .44 & 25 & .16 \\
\hline $0 i 1$ & 2 & .10 & 25 & .01 \\
\hline Mylar & 3 & .13 & 200 & .80 \\
\hline Polyethylene & 2 & .10 & 140 & .30 \\
\hline
\end{tabular}

The last column in the table represents the practical energy densities avallable with various dielectrics. Solld dielectrics such as mylar and polyethylene have greater capabilities with respect to energy densities than liquids, but as noted earlier, they also have very limited lifetimes under highly stressed, repetitive conditions. It was for this reason that a liquid was chosen as the energy storage medium for incorporating into a reliable, long-lived, high average power electrical driving network for UV laser excltation. 


\section{Pulse Shape}

Energy storage elements are not only sources of energy in a discharge circuit but also serve to help shape the output pulse. The storage element itself does not solely shape the output pulse but does so in conjunction with the load it sees on its output terminals. Capacitors are essentially made up of series and parallel combinations of either strip (i.e., parallel plate) or coaxial electrodes. There are three distinct types of pulse shapes that can be realized from such a network depending on the conditions that exist at the output of the storage element. Figure 3.2 shows the three voltage or current pulse shapes with the corresponding relationship between the $R$, $L$, and $C$ components of the circuit that must be met in order to achieve these pulse shapes.

Figure 3.2A shows the pulse shapes obtained when the electrical energy storage element acts as a conventional capacitive discharge system (lumped parameter networks). Figure 3.2B indicates the pulse shapes obtained using transmission lines for storage elements (distributed parameter networks). The difference between these two pulse shapes lies in the risetime of the discharge into the load as compared to the pulse length. A capacitive discharge has a risetime into the load of:(19)

$$
\mathrm{T}_{\mathrm{r}}=2 \cdot 2 \mathrm{~L}_{\mathrm{T}} / \mathrm{R}
$$

where $\mathrm{T}_{\mathrm{r}}$ is the risetime (10 to $90 \%$ ) into the load, $\mathrm{L}_{\mathrm{T}}$ the total 

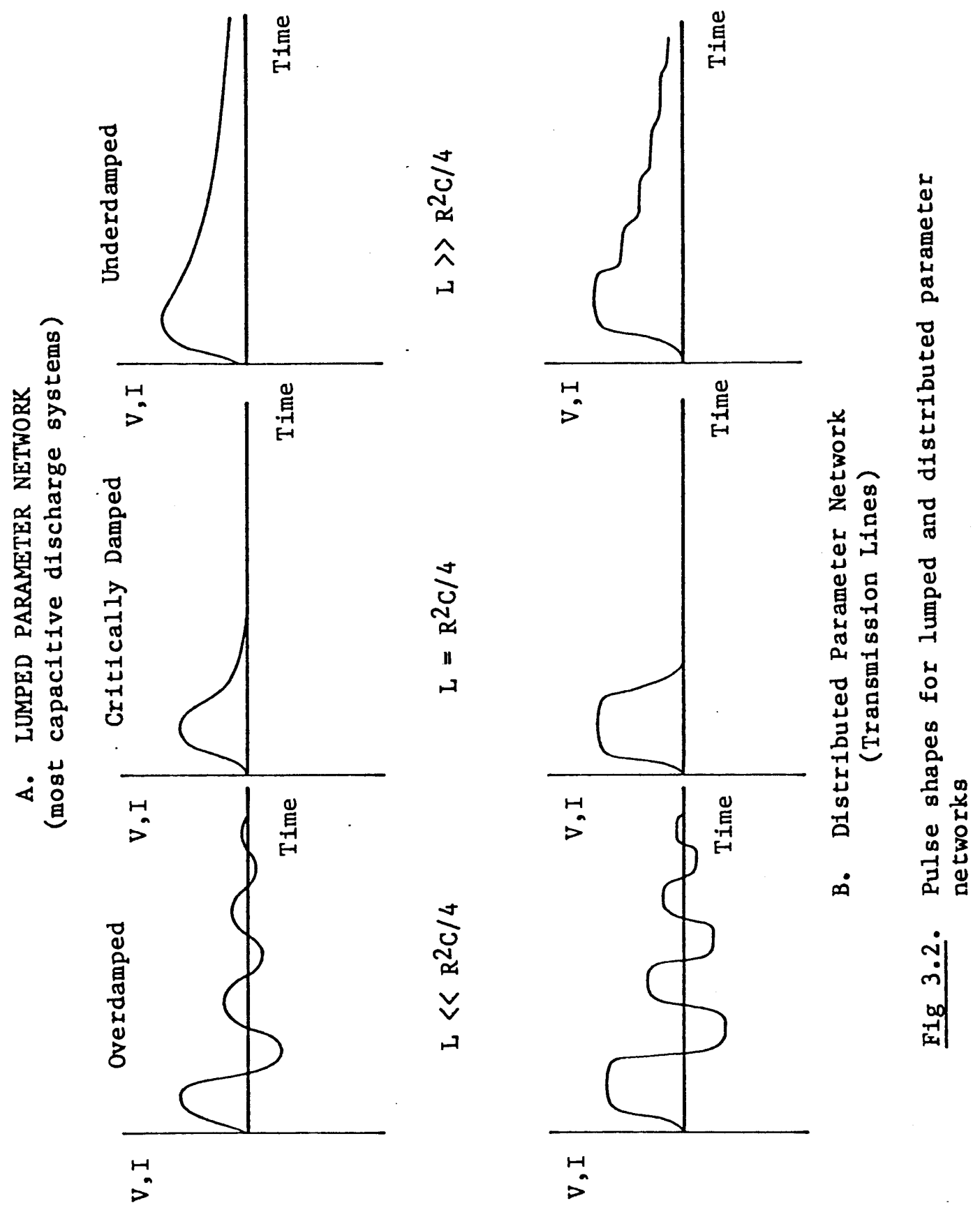
discharge loop inductance and $R$ is the load resistance. A transmission line has a risetime equal to (19).

$$
\mathrm{T}_{\mathrm{r}}=\mathrm{L}_{\mathrm{O}} / \mathrm{Z}_{\mathrm{TL}}
$$

with $L_{0}$ being the output inductance and $z_{0}$ the transmission line impedance. Comparing these two expressions, it should be noted that the risetime of a transmission line is proportional to the output inductance and not the total inductance of the discharge loop as in the capacitive system. The difference in these two expressions can be traced back to the pulse lengths obtained with these two types of networks. If the risetime of the network into the load is much less than half the one way transit time of the network, then the transmission line pulse shape will be observed (Figure 3.2B). On the other hand, if the risetime is much greater than the one way transit time of the network, then the lumped parameter pulse shape will be observed (Figure 3.2A). The one way transit time of a transmission line is the time it takes for a light pulse to travel from one end of the transmission line to the other through the dielectric. This pulse length versus risetime criterion distinguishes whether an energy storage element will act as a transmission line or a capacitor when discharged into some load. For a given output inductance the pulse shape of a transmission line approaches that of a capacitve system as its impedance is reduced. Theoretically, a transmission line discharged into an impedance-matched and completely resistive load should yield a perfectly rectangular pulse with no risetime associated with it. The inherent inductance in the bus work connecting the transmission line 
to the load and in the load itself modifies this ideal wave shape into one with a finite risetime.

Fast risetime, rectangular shaped pulses are highly desirable for driving many lasing gas mediums. If the driving or excitation pulse has a long risetime there is a significant amount of energy delivered to the load before lasing threshold is reached. This also holds true for the tail end of the pulse. Therefore, a rectangular wave shape would be ideal for maximizing the energy transfer between source and load during the lasing process. A fast risetime pulse is also desirable for systems that exhibit bottlenecking and selfterminating characteristics. A pulse with too slow a risetime can prevent lasing from occuring altogether in these types of system, so that it is imperative to use a fast risetime pulse for their excitation. The flat top characteristic of transmission lines also simplifies the conditions under which the lasing process takes place. This simplifies the study of not only kinetics but gain parameters as we 11 because of the constant driving force that exists during much of the time that lasing does occur.

A transmission line discharged into a matched resistive load delivers a voltage pulse of magnitude equal to one-half the charging voltage Vo. The current pulse is given by

$$
\begin{gathered}
I=v_{T L} /\left(R+z_{T L}\right)=v_{T L} / 2 z_{T L} \\
v_{O}=\left(v_{T L}\right) /\left(R+z_{T L}\right)
\end{gathered}
$$

The power delivered to the load is by circuit theory. 
In general,

$$
P_{0}=V_{0} I_{0}
$$

Substituting (23) and (24) in (25)

$$
\mathrm{P}_{\mathrm{O}}=\mathrm{V}_{\mathrm{TL}}{ }^{2} / 4 \mathrm{Z}_{\mathrm{TL}}
$$

and using the expression $E=1 / 2 \mathrm{CVo}^{2}$ from equation (6) for the energy stored in the transmission line, the pulse length can be solved for using the fact that $\mathrm{P}_{\text {out }}=\mathrm{E} / \mathrm{T}$,

where $T$ is the pulse length.

Thus

$$
\mathrm{T}_{\mathrm{TL}}=\left(\mathrm{C}_{\mathrm{TL}} \mathrm{V}_{\mathrm{TL}} 2 / 2\right) /\left(\mathrm{V}^{2} / 4 \mathrm{Z}_{\mathrm{TL}}\right)=2 \mathrm{C}_{\mathrm{TL}} \mathrm{Z}_{\mathrm{TL}}
$$

Using the fact that $\mathrm{Z}_{\mathrm{TL}}=(\mathrm{L} / \mathrm{C})^{1 / 2}$ (see footnote on page 18)

$$
\mathrm{T}_{\mathrm{TL}}=2 \mathrm{C}_{\mathrm{TL}}\left(\mathrm{L}_{\mathrm{TL}} / \mathrm{C}_{\mathrm{TL}}\right)^{1 / 2}=2\left(\mathrm{~L}_{\mathrm{TL}} \mathrm{C}_{\mathrm{TL}}\right)^{1 / 2}
$$

Note that this is always less than the time period of a critically damped capacitive discharge $T_{c}=T(L C)^{1 / 2}$. This is a natural result of the fact that a transmission line discharge only degrades into a capacitive shaped discharge pulse when the output inductance increases beyond a certain point. This increase in the output inductance not only changes the shape of the pulse but also increases the time period in which energy flows to the load. Therefore, one of the primary design criterion in the selection of the liquid dielectric and electrode structure was that the resulting electrical energy storage element act as a transmission line in the electrical discharge circuit. 


\section{CHARGING CIRCUITRY}

Introduction

After the decision was made to design and construct a liquid dielectric energy storage element that would act as a transmission line when discharged into an appropriate load, it was also necessary to design a charging circuit that would transfer energy from the laboratory power supply to the energy storage elements of the high speed circuit itself. The three types of charging networks that will be discussed in the next section include resistive, inductive and inductive-diode charging circuits.

In any fast discharge circuit there always exists an element for isolating the power supply from the energy storage element during the fast discharge phase of operation. The necessity of this element evolves out of the fact that most laboratory power supplies cannot furnish on a continuous basis the extremely high levels of power needed to pump many types of UV lasers. Therefore, these types of devices are usually operated in the pulsed mode for this and other reasons (i.e. self-termination). Figure (3.1) previously showed the conventional arrangement of components in such a circuit.* In this instance, a capacitor is charged up until the output switch closes and connects the capacitor directly across the load. The output switch can operate in either a triggered or self-breakdown mode. When switch closure does

\footnotetext{
ॠNotice that in all the circuits used, at least one side of the highvoltage network is always grounded. This reduces the number of points in the circuit that are at high voltage and is a typical safety precaution in high-voltage systems.
} 
occur, the capacitor delivers its energy to the load very quickly in any properly designed circuit. After delivery, the capacitor voltage returns to zero so that point $A$ without the isolating element would present a short-circuit to the power supply causing either serious damage to the supply by overloading or decoupling by protective fuse or relay openings. At a given voltage the isolating element must always present an effective impedance to the supply such that the maximum current drawn does not exceed the supply capabilities.

\section{Resistive Charging}

Resistive charging is probably the most widely used charging technique because of its simplicity, low cost and availability of components. Figure $4.1 \mathrm{a}$ show the conventional arrangements of components in the charging circuit while Figure $4.1 \mathrm{~b}$ shows the operational waveforms. Initially, the capacitor is fully discharged and the switch is open. The subsequent capacitor voltage and current will then be(8)

$$
\begin{aligned}
& v(t)=V\left[1-e^{-(t / R C)}\right] \\
& i(t)=(V / R)\left[e^{-(t / R C)}\right]
\end{aligned}
$$

The energy delivered by the power supply $\left(E_{S}\right)$ during a time period $T$ is just

$$
E_{S}=v \int_{0}^{T} i(t) d t
$$

Substituting (30) in (31) and integrating

$$
E_{S}=C V^{2}\left[1-e^{-(T / R C)}\right]
$$


The energy stored in the capacitor $\left(E_{c}\right)$ at this time $T$ is

$$
E_{c}=1 / 2 \operatorname{Cv}(T)^{2}
$$

Substituting (29) in (33), the energy stored in the capacitor at time $T$ becomes

$$
E_{C}=1 / 2 C V^{2}\left[1-e^{-(T / R C)}\right]
$$

The energy transfer efficiency between source and capacitor is just the ratio of the energy stored in the capacitor to that delivered by the source in the same time period, or

$$
=1 / 2 \quad\left[1-e^{-(T / R C)}\right]^{2}
$$

Clearly the maximum efficiency for resistive charging is only $50 \%$ and this occurs only when $\mathrm{T} \longrightarrow \infty$ or $\mathrm{RC} \longrightarrow 0$. These two conditions are never met in any practical situation and especially those cases pertaining to high repetition rate operation. Because high repetition rates are a desired goal, equation (35) must be evaluated in order to determine the practical working efficiencies attainable under high repetiion rate conditions. Since it is also desired to maximize the power transfer from the source to the load, expression (34) should be manipulated in such a fashion as to yield the condition under which maximum power transfer occurs. This yields the following equation:

$$
E_{c}=1 / 2 \operatorname{cV}^{2}\left[1-e^{-(T / R C)}\right]^{2}
$$

For a given supply voltage $V$ and repetition rate, $T$ and $R$ will remain 


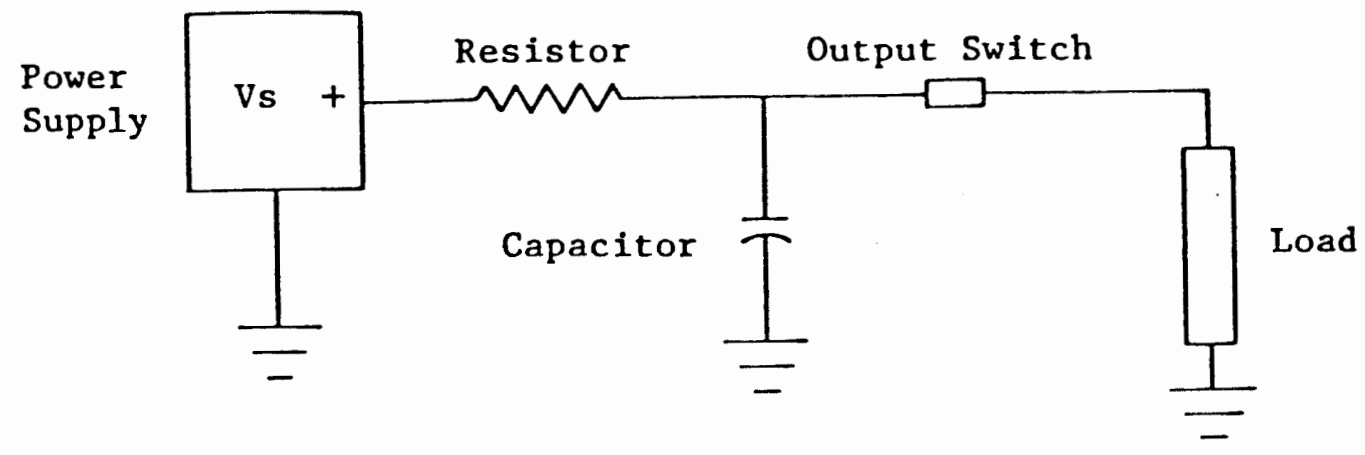

Fig 4.1a. Resistively charged circuit

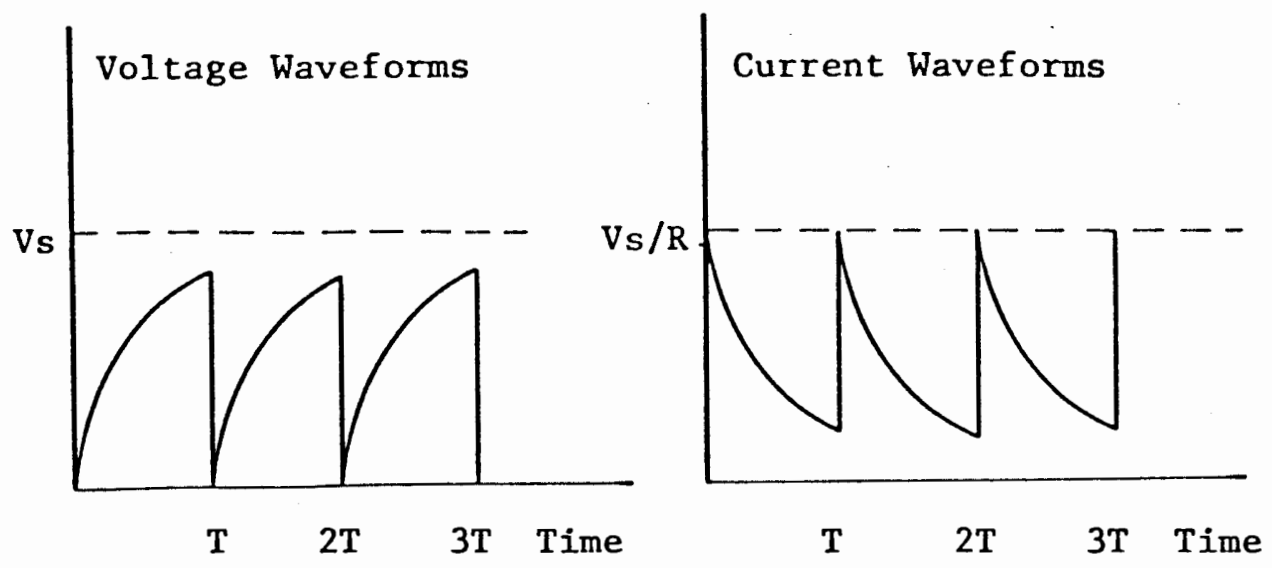

Fig 4.1b. Capacitor voltage and current waveforms in a resistively charged circuit 
fixed. $R$ has to remain fixed because for a given supply voltage the maximum current drawn has to be within the supply range. Therefore, the only remaining variable is the capacitance $C$. To find the maximum of the power that could potentially be transferred to the load $\mathrm{P}_{\mathrm{L}}$, it is simply a matter of differentiating (34) with respect to $\mathrm{C}$, setting the resulting equation to zero and then solving for the relationship between the independent (C) and dependent $(R, T)$ variables. Letting $\left(P_{L}\right)_{\max }$ equal the maximum power that could be transferred to the load and using this procedure yields the following equation*.

$$
C=(R / 2 T)\left[e^{(T / R C)}-1\right]-1
$$

Inspection of this equation will show that it is transcendental in nature and an exact solution does not exist. Nevertheless, an approximate solution can be found*.

$$
C=.73 T / R
$$

Substitution of (37) in (33) and (34) yield an expression for the maximum transfer efficiencies attainable using a resistive charging network under high repetition rate conditions.

$$
\begin{gathered}
\left(P_{0}\right)_{\max }=T^{2} / 5 R \\
\eta=37 \%
\end{gathered}
$$

\footnotetext{
*Appendix 1
} 
Therefore, with high repetition rates and resistive charging almost two-thirds of the source power is unavailable at the load. This clearly demonstrates the need for alternate and more efficient methods for charging the energy storage element in high pulse rate circuits. In fact, the most efficient types of charging networks are those which utilize resonance techniques.(20) Rather than using a resistive isolating element, they employ an inductive element. This technique minimizes the resistances of the charging circuit with the result that high transfer efficiencies are possible between source and storage element than can be achieved with resistive charging schemes.

\section{Resonant Inductive Charging}

Figure 4.2a illustrates the inductive charging circuit and Figure $4.2 \mathrm{~b}$, the waveforms of a circuit utilizing an inductive charging element. In order to maximize transfer efficiencies, the resistive component of such a circuit should be as small as possible (i.e. the circuit should operate in an underdamped or oscillatory mode).* The condition for this is defined by

$$
R \ll 2 \pi(L / C)^{1 / 2}
$$

Also to prevent significant voltage droop during charging, the source capacitance should be at least 10 times larger than that of the storage element. The series resistance in the charging circuit (see Figure 4.4)

*Note that this criterion is different for a system charging a capacitive load than one charging a resistive load in which a critically damped oscillation is the optimum one. 
is usually just the inherent or series resistance in the coil windings of the inductor. In commercially avallable inductors, this resistance is of low enough magnitude that transfer efficiencies greater than $90 \%$ are common. If the triggering sequence of the output switch is such that it keeps in step with the resonant transfer of energy from the source to storage element, the following condition holds true.

$$
\mathrm{T}_{\mathrm{R}}=\pi\left(\mathrm{L}_{\mathrm{c}} \mathrm{C}_{\mathrm{s}}\right)^{1 / 2}
$$

Where $\mathrm{L}$ is the charging inductance, $\mathrm{C}$ the storage element capacitance and $\mathrm{T}_{\mathrm{R}}$ is the resonant charging time.

It is useful to develop several design equations by which the average current capability of the power supply ( $I_{\text {ave }}$ ) can be related to the usable values of the charging inductance, energy storage capacitance and desired repetition rate. Consider first the average current drawn from the source.

$$
\mathrm{I}_{\mathrm{ave}}=\mathrm{Q} / \mathrm{T}
$$

where $Q$ is the total charge transferred during the charging period $T$.

$$
\mathrm{Q}=\mathrm{CV}_{\mathrm{C}}
$$

with $V_{C}$ being the capacitor voltage and $C$ the storage element capacitance.

During resonant charging a voltage doubling occurs. (18) This is an important attribute of resonant charging in that for a 
given output voltage only half as much supply voltage is required. Therefore

$$
Q=2 \mathrm{CVs}
$$

where $V_{S}$ is the supply voltage. Therefore

$$
I_{\text {ave }}=2 \mathrm{CV}_{\mathrm{s}} / \mathrm{T}
$$

Solving for the capacitance, we arrive at one design equation that is

$$
\mathrm{C}=\mathrm{I}_{\mathrm{ave}} \mathrm{T} / 2 \mathrm{~V}_{\mathrm{s}}
$$

Eliminating $C$ in (47) by using equation (42) and solving for the inductance, we find

$$
L=2 T V_{s} / T T^{2} I_{\text {ave }}
$$

The energy stored in the pulse forming network after charging is

$$
\mathrm{E}=2 \mathrm{CV}_{\mathrm{s}}^{2}
$$

It should be noticed that in Figure 4.5 the pulse forming network (PFN) or energy storage element is discharged at the time of peak voltage. If the system was not discharged at this time, the flow of energy would reverse itself and flow backwards from the PFN to the source. Therefore, the PFN should be discharged at or near the peak system voltage in order to optimize energy transfer from source to PFN. This mode of operation with a given charging inductor and PFN capacitance will necessarily limit the repetition rate to a single frequency determined by the resonant frequency of the charging circuit. 
A wider range of operational frequencies can be obtained by triggering the discharge on the rising portion of the PFN voltage voltage. This is the so called "linear" charging range in that the wave front is approximately linearly increasing with time.(26) The cost of operating in the linear charging range as opposed to resonant charging is illustrated in Figure 4.7. It should be evident that for a given amount of switch jitter the possible variations in output voltage are much larger in linear charging because of the difference in the slope of the wavefront at the time of discharge. It also should be added that the transfer efficiency will be lower in the linear case thus detracting from its advantage of having a wider range of repetition rates than resonant charging. One simple, inexpensive modification that can significantly widen the operational frequencies of a resonant charging circuit is the addition of a high voltage chain of diodes in series with the charging inductor as shown in Figure 4.6. The high-voltage chain of diodes prevents the PFN from discharging back into the source during the course of a normal resonant charging cycle. Figure 4.8 illustrates the waveforms attained with this type of resonant charging circuit. $T$ is the time period between discharge pulses while $T_{R}$ is just the resonant charging time. It can be seen from the above figures that $T$, the time between discharges can be extended out to infinity if desired limited only by the leakage in the diode chain and dielectric. Since the pulse recurrence frequency or repetition rate is inversely proportional to this time (PRF $\mathrm{T}^{-1}$ ) the repetition rate can span a range from zero pps (pulses per second) all the way up to twice the resonant charging frequency ( $i . e . f=2 f o$ ) 
and beyond if linear charging is acceptable. This technique of inductor-diode charging is probably the most commonly used because of the wide range of repetition rates available as well as the high transfer efficiencies attainable.

The actual transfer efficiency is given by the expression(21)

$$
\eta=1-\pi / 4 Q
$$

where $Q$ is the quality factor of the charging circuit. The quality factor is given by

$$
Q=\omega L / R
$$

where $W$ is the angular oscillation frequency, and $R$ is the total series resistance in the charging circuit.

Quality factors from ten to twenty are common in such circuits with efficiencies from $92 \%$ to $95 \%$ being readily achieved. In summary, of all the charging schemes, resonant inductor charging with a series diode represents one of the most efficient charging techniques which also encompasses a fairly wide range of operational frequencies.(21) Another important characteristic of resonant charging is in its ability to double the input voltage. This relaxes the requirements of the power supply for a given output voltage. Resistive charging which has been shown to be highly inefficient under high repetition rate conditions should only be used in low power circuit and applications in which inefficiencies are unimportant. 


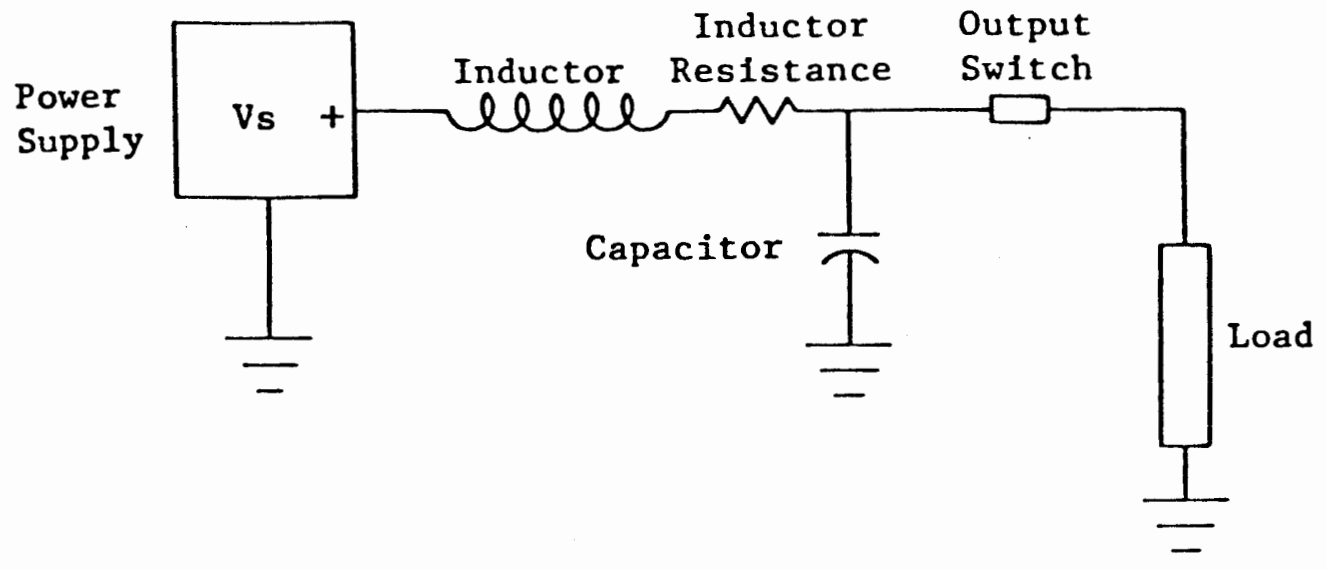

Fig 4.2a. Inductively charged circuit

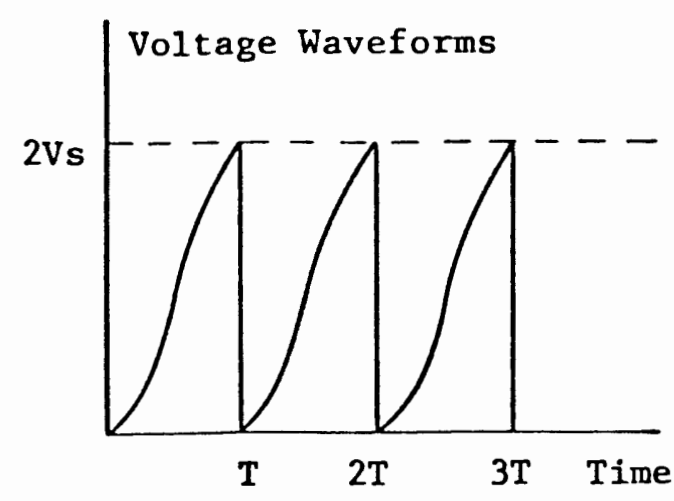

Current Waveforms

Fig 4.2b. Capacitor voltage and current waveforms in an inductively charged circuit
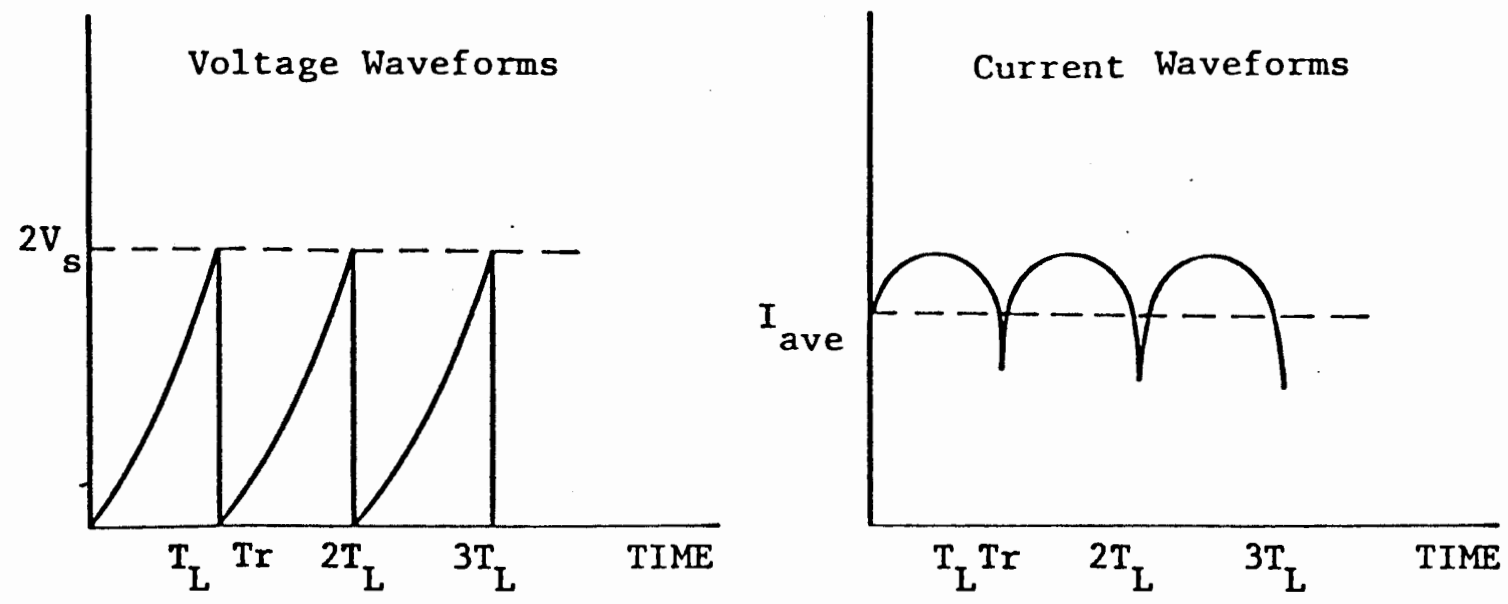

Fig 4.3. Capacitor voltage and current waveforms in the linear charging range 


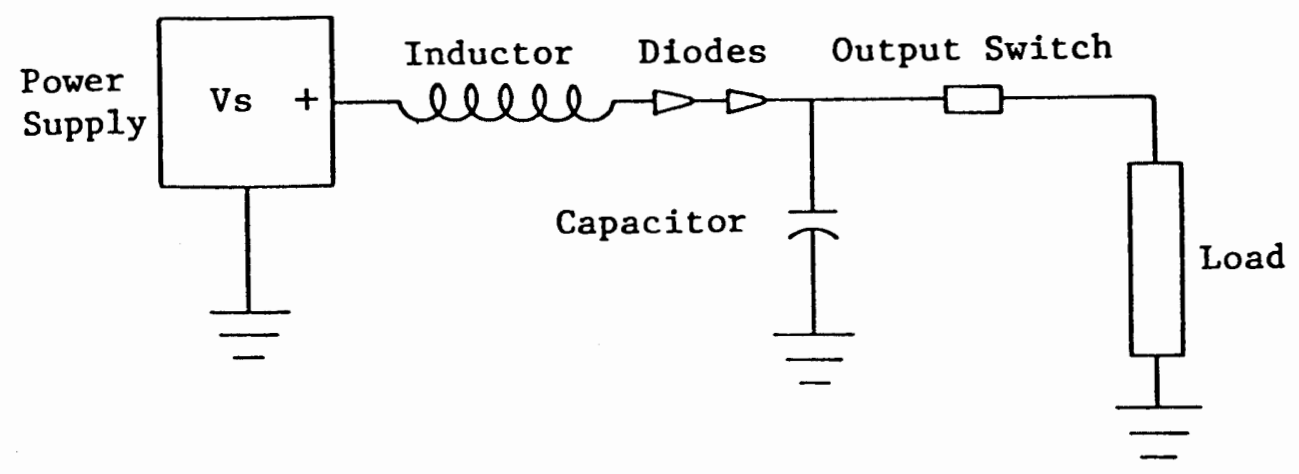

Fig 4.4. Inductor-diode charged circuit

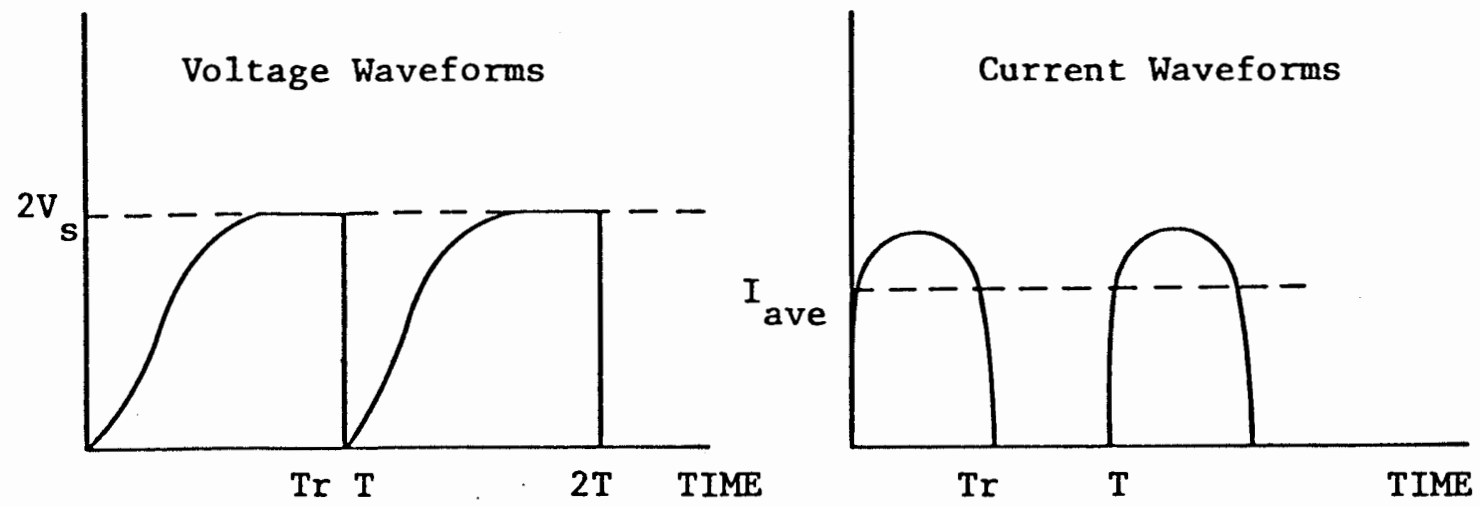

Fig 4.5. Capacitor voltage and current waveforms in an inductor-diode charged circuit 


\section{HIGH POWER PULSER DESIGN}

Transmission Line Geometry and Power Transfer

The design of the present high power pulser electrical discharge circuit is based on the use of liquid filled transmission lines (LTL). Glycerine was selected for use because of its dielectric constant $(K=44)$, dielectric strength $(>20 \mathrm{kv} / \mathrm{mm})$ and its electrical stability under no flow conditions. In any fast discharge circuit there are several design parameters that must be considered before the circuit is constructed. These are output energy, pulse shape and length, and load impedance. The first and the third parameters determine the maximum power input to the load when the source and load impedance are matched. In order to optimize conversion efficiencies, as well as reduce the variation in the driving force during the course of the discharge, it was decided to generate rectangular shaped pulses by the use of transmission lines. The pulse length was selected to be approximately 50 nsec because it represents a fairly optimal pulse length for many self-terminating UV lasers as well as keeping the length of the apparatus down to a reasonable size. The actual length will depend upon the speed of light in the dielectric medium used in the transmission lines. The speed of light in glycerine is given by the expression

$$
c_{k}=c_{v} / k^{1 / 2}
$$

Where $c_{k}$ is the speed of light in a medium of dielectric constant $k$ and $c_{v}$ is the speed of light in a vacuum. Solving for $c_{k}$ 


$$
c_{k}=\left(3 \times 10^{10} \mathrm{~cm} / \mathrm{sec}\right) /(44)^{1 / 2}=4.52 \times 10^{9} \mathrm{~cm} / \mathrm{sec}
$$

Since the pulse length is the two-way transit time in the medium of interest (19)

$$
c_{\mathrm{pk}}=2.26 \times 10^{9} \mathrm{~cm} / \mathrm{sec}
$$

where $c_{p k}$ is the pulse velocity in a medium of dielectric constant $k$. Solving for the desired transmission line length using $50 \mathrm{nsec}$ as the desired pulse length.

$$
\mathrm{L}_{\mathrm{t}}=\left(\mathrm{c}_{\mathrm{pk}}\right)(50 \mathrm{nsec})=113 \mathrm{~cm}=1.13 \mathrm{~m}
$$

The actual length of the constructed transmission lines is $122 \mathrm{~cm}$, which gives an output pulse length $\left(\mathrm{T}_{\mathrm{p}}\right)$ of

$$
\mathrm{T}_{\mathrm{p}}=\left(1.22 \times 10^{2} \mathrm{~cm}\right) /\left(2.26 \times 10^{9} \mathrm{~cm} / \mathrm{sec}\right)=54 \mathrm{nsec}
$$

It should be apparent that for pulse lengths approaching, say a usec the device length would be over 74 feet. A device this size would have serious construction problems associated with it, especially those concerned with keeping a constant impedance over its entire length. It would be far simpler to utilize lumped parameter networks for pulse lengths this long.

The next parameter to be established is the output pulse energy. A value of 1 joule was initially chosen as a design point for several reasons. First, this represents enough energy to successfully drive many types of UV lasers.(22) Second, 1-joule per discharge 
would minimize the electrode loading hopefully extending their useful lifetimes at high repetition rates. With this in mind, recall that the energy stored in a transmission line is by (6) equal to

$$
\mathrm{E}_{\mathrm{TL}}=1 / 2 \mathrm{cV}^{2}
$$

The capacitance $\mathrm{C}_{\mathrm{TL}}$ of the transmission line is given by (11)

$$
\mathrm{C}_{\mathrm{TL}}=\epsilon \mathrm{wL} / \mathrm{s}=44 \epsilon_{\mathrm{owLo}} / \mathrm{s}
$$

The device length has already been determined by the output pulse length and is equal to $122 \mathrm{~cm}$. Therefore

$$
C=(5368 \in 0) w / s
$$

For practical purposes of material selection, an electrode width of 1/2-inch was used.

$$
C=\left(6817 \in_{0}\right) / s
$$

The only remaining parameter is the separation distances. The last parameter's value can be determined from consideration of the load impedance. In order to maximize power transfer, the source impedance should be matched to the load impedance. It was determined from available data that a load impedance of 1 ohm was a realistic value for many types of discharges.(9) The impedance of a transmission line $\left(z_{T L}\right)$ is given by the expression (21) 


$$
\mathrm{z}_{\mathrm{TL}}=377 \mathrm{~s} / \mathrm{k}^{1 / 2} \mathrm{w}
$$

Using a value of $1 \mathrm{ohm}$ for the load and transmission line impedances, the separation distance can be solved for because all the remaining variables, including the dielectric constant $\mathrm{K}$ and line width $\mathrm{w}$ are given. Therefore

$$
\begin{aligned}
& \mathrm{s}=\mathrm{z}_{\mathrm{TL}} \mathrm{k}^{1 / 2} \mathrm{w} / 377 \\
& \mathrm{~s}=(1 \Omega)(44)^{1 / 2}(1.27 \mathrm{~cm}) /(377 \Omega) \\
& \mathrm{s}=.02 \mathrm{~cm}
\end{aligned}
$$

Substituting this value in (53) and solving for the capacitance $C$.

$$
\begin{aligned}
& \mathrm{C}=6817 \epsilon \mathrm{o} / .02 \mathrm{~cm} \\
& \mathrm{C}=(6817)(8.85 \mathrm{uf} / \mathrm{cm}) /(.02 \mathrm{~cm})\left(10^{8}\right)=.03 \mathrm{uf} \\
& \text { or } \quad \mathrm{C}=30 \mathrm{nf}
\end{aligned}
$$

Solving now for the voltage required to produce a 1 joule output pulse.

$$
\begin{aligned}
E & =1 / 2 C V^{2} \\
\text { or } V & =(2 E / C)^{1 / 2} \\
V & =(2 j / 30 n f)^{1 / 2}=8165 \text { volts }
\end{aligned}
$$

A quick check of the dielectric stress at these voltage levels for the prescribed separation distances will show that it exceeds the dielectric breakdown strength of glycerine. Therefore, it was necessary to increase the total capacitance of the transmission line by paralleling the three transmission lines in the present apparatus and operate into 
a slightly mismatched mode in order to obtain 1 joule in the primary output pulse*. From a power delivering standpoint, a certain amount of mismatch can be tolerated without delivering a significant part of the stored energy after the main output pulse. Recall that the power output $\mathrm{P}_{\mathrm{O}}$ is

$$
P_{0}=I_{0} V_{0}
$$

$$
\begin{array}{lll} 
& \text { where } & \mathrm{I}_{\mathrm{O}}=\frac{\mathrm{V}_{\mathrm{TL}}}{\mathrm{Z}_{\mathrm{TL}}+\mathrm{R}} \\
\text { and } & \mathrm{V}_{\mathrm{O}}=\frac{\mathrm{V}_{\mathrm{TL}} \mathrm{R}_{\mathrm{L}}}{\mathrm{Z}_{\mathrm{TL}}+\mathrm{R}_{\mathrm{L}}}
\end{array}
$$

Operating into a matched load, these expressions reduce to

$$
\mathrm{I}_{\mathrm{o}}=\mathrm{V}_{\mathrm{O}} / 2 \mathrm{Z}_{\mathrm{TL}} \text { and } \mathrm{V}_{\mathrm{O}}=\mathrm{V}_{\mathrm{TL}} / 2
$$

Therefore the maximum power output will be

$$
\left(\mathrm{P}_{\mathrm{o}}\right)_{\max }=\left(\frac{\mathrm{V}_{\mathrm{TL}}}{2 \mathrm{Z}_{\mathrm{TL}}}\right)\left(\frac{\mathrm{V}_{\mathrm{TL}}}{2 \mathrm{Z}_{\mathrm{TL}}}\right)=\frac{\mathrm{V}_{\mathrm{TL}} 2}{4 \mathrm{Z}_{\mathrm{TL}}}
$$

The power output into a mismatched load will be

$$
\mathrm{P}_{\mathrm{O}}=\left(\frac{\mathrm{v}_{\mathrm{TL}}}{\mathrm{z}_{\mathrm{TL}}+\mathrm{R}_{\mathrm{L}}}\right)\left(\frac{\mathrm{z}_{\mathrm{TL}} \mathrm{R}_{\mathrm{L}}}{\mathrm{Z}_{\mathrm{TL}}+\mathrm{R}_{\mathrm{L}}}\right)=\frac{\mathrm{v}_{\mathrm{TL}}{ }^{2} \mathrm{R}_{\mathrm{L}}}{\mathrm{Z}_{\mathrm{TL}}+\mathrm{R}_{\mathrm{L}}}
$$

*Increasing the capacitance allowed the separation distanced to be increase thereby increasing the effective breakdown strength while still keeping the impedance constant. 
Taking the ratios of these two power outputs

$$
\mathrm{Po} / \mathrm{P}_{\mathrm{o}}=\frac{\mathrm{V}_{\mathrm{TL}}{ }^{2} \mathrm{R}}{\left(\mathrm{Z}_{\mathrm{TL}}+\mathrm{R}\right)^{2}} \quad \frac{\mathrm{V}_{\mathrm{TL}}{ }^{2}}{4 \mathrm{Z}_{\mathrm{TL}}}=\frac{4 \mathrm{Z}_{\mathrm{TL}} \mathrm{R}_{\mathrm{L}}}{\mathrm{Z}_{\mathrm{TL}}{ }^{+} \mathrm{K}_{\mathrm{L}}} 2
$$

Let the load impedance $R_{L}$ be given as a fraction $x$ of the transmission line impedance

$$
\text { i.e. } \mathrm{x}=\mathrm{R}_{\mathrm{L}} / \mathrm{Z}_{\mathrm{TL}} \text { or } \mathrm{R}_{\mathrm{L}}=\mathrm{x} \mathrm{Z}_{\mathrm{TL}}
$$

Substituting this expression in (59) and reducing

$$
\begin{aligned}
& \mathrm{Po} / \mathrm{Po}_{\max } \frac{=4 \mathrm{Z}_{\mathrm{TL}}\left(x \mathrm{Z}_{\mathrm{TL}}\right)}{\mathrm{Z}_{\mathrm{TL}}+\left(\mathrm{xZ_{ \textrm {TL } } ) ^ { 2 }}\right.}=\frac{4 x \mathrm{Z}_{\mathrm{TL}}{ }^{2}}{(1+\mathrm{x}) \mathrm{Z}_{\mathrm{TL}}{ }^{2}} \\
& \mathrm{P}_{\mathrm{o}} / \mathrm{P}_{\mathrm{O}_{\max }}=\frac{4 \mathrm{x}}{(1+\mathrm{x})^{2}}
\end{aligned}
$$

As an example, assume that it is desired to deliver at least 75 percent of the total available energy in the main pulse. Enter this value for $\mathrm{P}_{\mathrm{O}} / \mathrm{P}_{\mathrm{omax}}$ and solve for $\mathrm{x}$ :

$$
\mathrm{x}=.33 \text { or } \mathrm{x}=2.80
$$

If the load impedance is $1 \mathrm{ohm}$, then a transmission line impedance of either 3.3 or .36 ohms could be used with only a 25 percent loss of energy during the main pulse. It is always advisable to operate in a high to low impedance mode because more energy can be stored in the higher impedance line (almost two magnitudes more). This is a result of the fact that as the impedance increases for a given TL geometry, 
the plate separation and the hold-off voltage also increases in the same ratio. On the other hand, the energy storage increases in quadrature with the hold-off voltage. Therefore, a doubling of the impedance and hold-off voltage will lead to a four-fold increase in the energy storage capability of the TL. Another advantage of using the higher impedance line is that since the pulse risetime is inversely proportional to the line impedance, a $3.3 \mathrm{ohm}$ line can not only deliver more energy in the first pulse, but its risetime will be 30 percent of what could be achieved under a matched load situation. The only trade-off is a reduction in primary pulse transfer efficiency. Figure 5.1 is a graph of power transfer efficiencies versus load-line ratio. Figure 5.2 shows a diagram of the transmission lines constructed on the basis of the previous analysis. Copper straps were used to bus the three upper electrodes together to form an effective electrode width of one and one-half inches.

\section{Pulse Charging Circuits}

Liquid dielectrics such as glycerine and water while possessing characteristics favorable to their use in energy storage schemes do suffer from the effects of non-zero electrical conductivities. Because of the finite conductivities associated with all dielectrics, there always exists a certain amount of internal bleed-off charge occurring once the dielectric has been energized. The speed by which this bleed-off occurs is usually expressed by the internal RC decay constant of the dielectric. At one time constant only 40 percent of the 


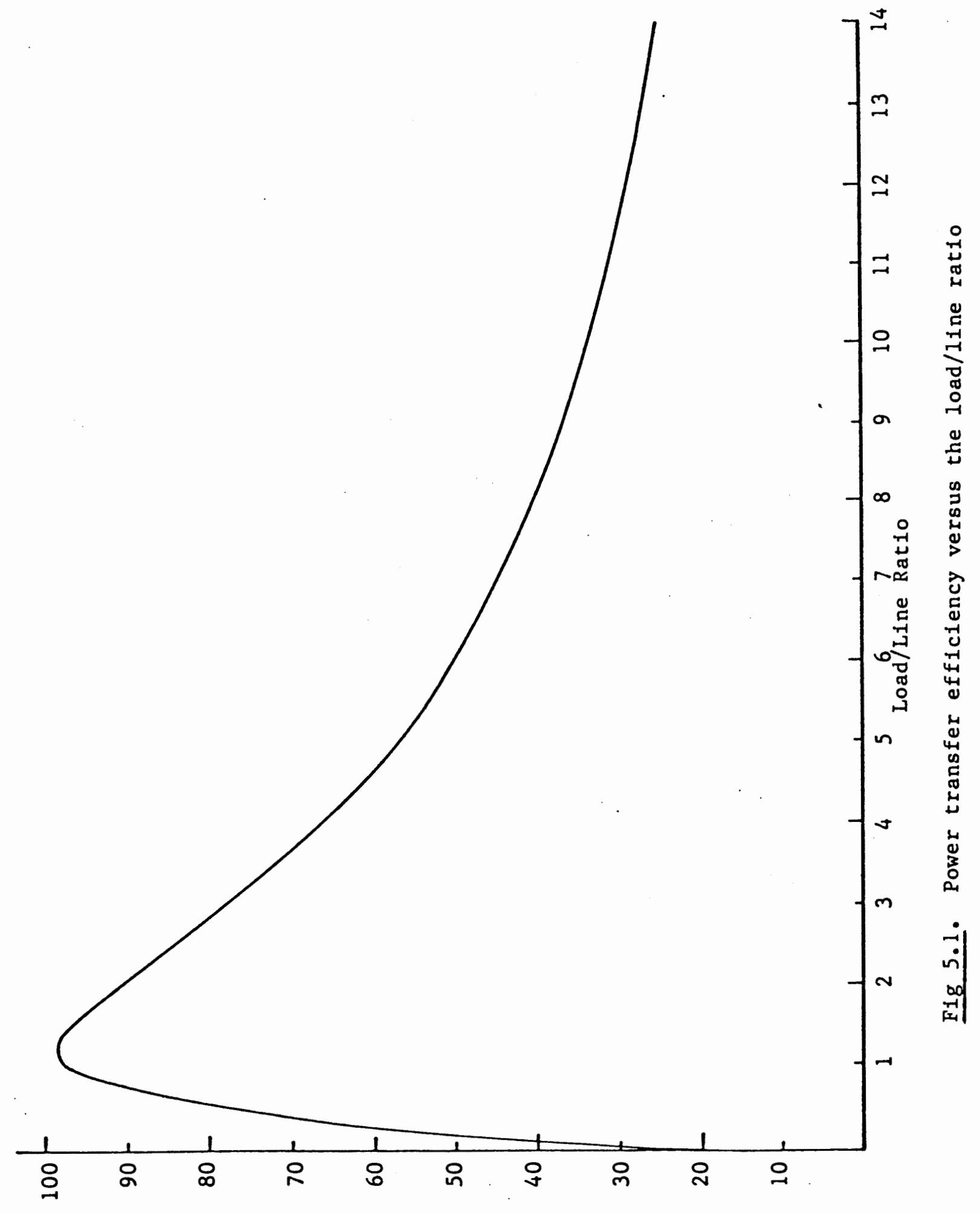

点矛 


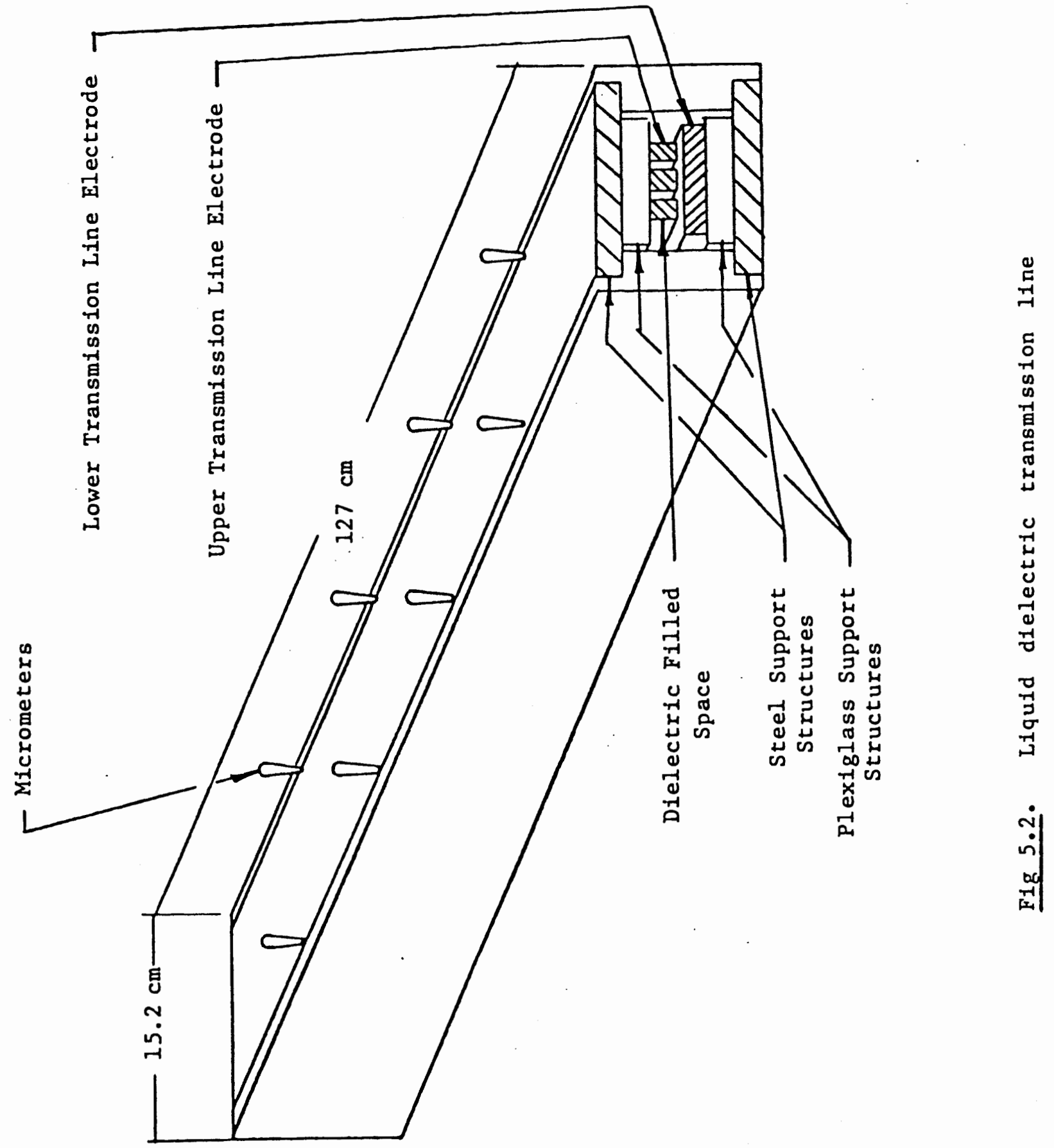


original amount of energy is left. The shorter the decay constant the faster bleed-off is occurring. The RC decay constant is equal to, the product of the resistivity and the dielectric permittivity.(11)* As an example of the time frames involved, consider the case of reasonably pure water having a typical resistivity of $1 \mathrm{M} \Omega-\mathrm{cm}$ and a permittivity of $80 \epsilon_{0}$. The RC decay time in this instance will then be about 7.8 usecs. It should be clear that optimal energy use requires fast pulse charging of the liquid filled transmission lines. If one desires that losses during charging amount to no more than 10 percent, then the required charging time can be determined.

$$
\begin{aligned}
& V(t)=V_{O} e^{-t / R C} \\
& E \propto V(t)^{2}
\end{aligned}
$$

Letting $\mathrm{RC}=7.8$ usecs and $\left(\mathrm{V}(\mathrm{t} / \mathrm{VO})^{2}=.9\right.$ then the $\mathrm{RC}$ decay constant can be determined to be .41 usec. Therefore, the charging time should be no more than $1 / 2$ usec in order to minimize losses during the charging cycle, hence the need for fast pulse charging circuits. A typical charging circuit utilizing a conventional solid dielectric capacitor only has to be charged a little faster than its discharge repetition rate. At a repetitive rate of $\mathrm{l} \mathrm{khz}$ the necessary charging time is only $1 \mathrm{msec}$ and is about 2,000 times slower than those required in liquid dielectric charging circuits.

The above case has been used to show the need for a fast pulse charging circuit in order to energize the liquid dielectric storage elements in the proper time frame. To this end there were two 
pulse charging circuits built and tested to verify that they could meet the above criterion. The first consisted of a two-stage Marx Bank, shown in Figure 5.3. A Marx Bank consists of a number of capacitors charged in parallel and discharged in series. Because of this parallel-series mode of operation, a Marx-Bank realizes an output voltage which is equal to the charging voltage $V_{i n}$ multiplied by the number of stages $N$ discharged in series (i.e. $v_{\text {out }}=N v_{\text {in }}$ ). (19) A twostage Marx Bank will then be a voltage doubling circuit with an output capacitance equal to one-half the stage capacitance. The reduced capacitance on the output stage is a result of the conservation of energy and because capacitors discharged in series must acquire the same amount of charge.(7) The present Marx Bank has an output capacitance of about $53 \mathrm{nf}$ with a maximum output voltage of $34 \mathrm{kv}$. This circuit was resistively charged because of the lack of an appropriate power supply and charging inductor. Later, funding allowed purchase of a $20 \mathrm{kv}, 250 \mathrm{ma}, 5 \mathrm{kw}$, power supply along with a 5 hy charging inductor. This allowe the use of a resonant charging technique to charge a single low inductance capacitor which was then switched in series with the liquid transmission lines by a jet blown spark gap. (Figure 5.3).(9) As discussed earlier, resonant techniques are very efficient energy users. This is the main reason for replacing the Marx Bank by a resonant scheme resulting in almost a ten-fold increase in efficiency. Most of this increase is related to the resonant versus resistive charging losses under high repetition rate conditions ( 7 percent for the resonant scheme and 64 percent for the resistive one). A second contributing factor is the ratio of the 
source capacitance to the line capacitance for any charging configuration. If the source capacitance is too large (ratio > l) there will be a late and inefficlent flow of energy through the load after the passage of the main pulse. If this capacitance is too small (ratio< 1) there will be a significant voltage droop occurring during charging of the transmission 1ines. The most optimal ratio exists when source and line capacitances are approximately equal (1.e. ratio = 1).(23) In the case of the Marx Bank it was the voltage droop during charging that was minimized by the use of a large capacitance ratio (53 nf versus 5 to $15 \mathrm{nf}$ ). In the resonant scheme only one $15 \mathrm{nf}$ capacitor was used allowing capacitances to be matched and thereby minimizing late and undesirable energy delivery. Even undesirable energy de11very. Even though some voltage droop does occur it is more important to make the best use of the avallable power than to achieve the highest output voltage. The amount of voltage droop occurring during such a charging cycle is:(23)

$$
\Delta V / V=\Delta E / 2 E
$$

Therefore, a 50 percent energy removal from the capacitor will result in a transmission line voltage of 75 percent the original voltage on the charging capacitor.

\section{Charging Network}

The equations derived in the section on pulse charging circuits will be used here to size various circuit components given the 


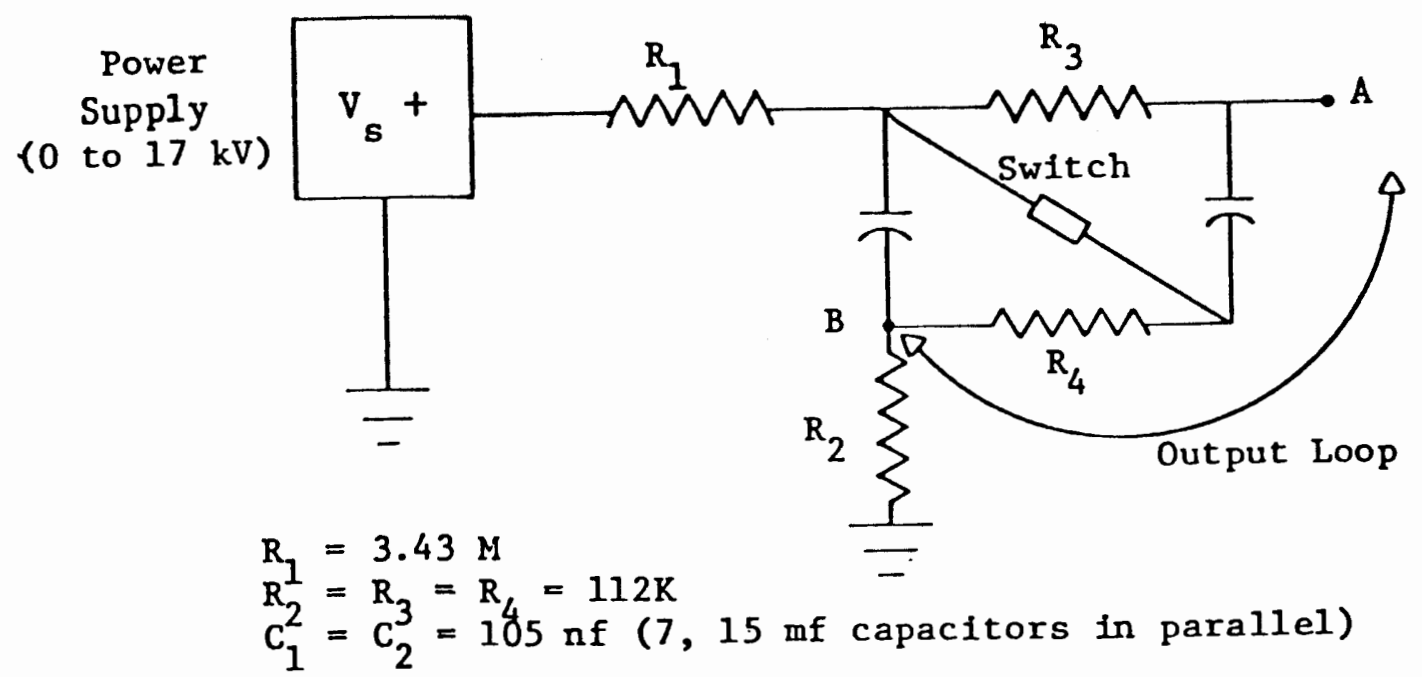

Fig. 5.3. Marx-Bank and charging circuit schematic

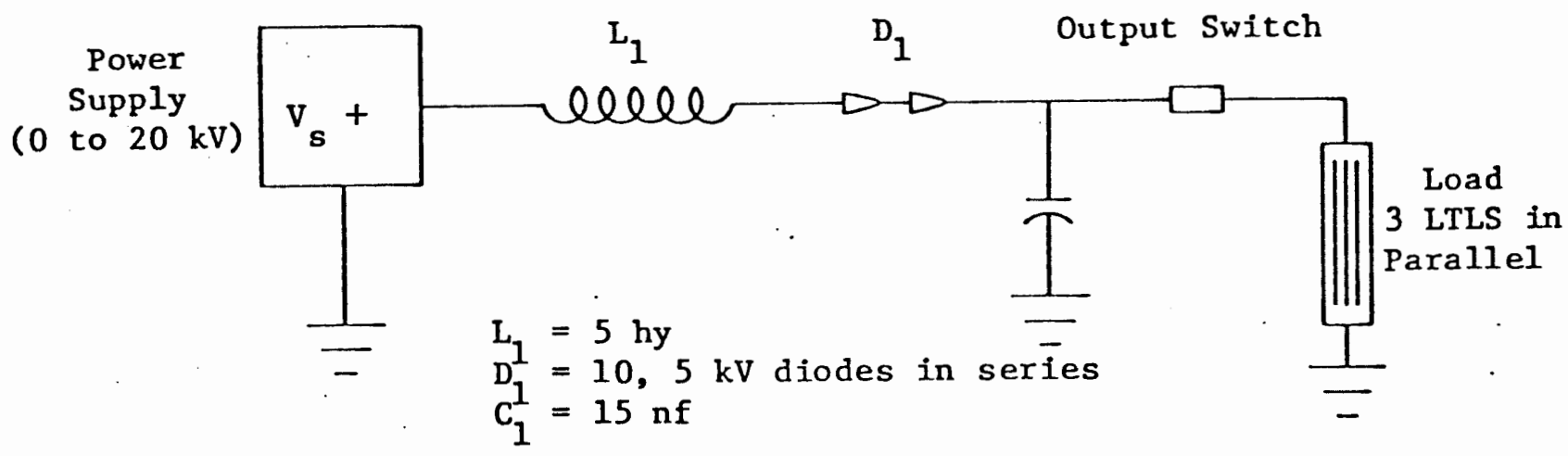

Fig. 5.4. Resonant charging circuit 
charging circuit type and certain dependent system parameters. The dependent circuit parameters will be the average supply current and the pulse recurrence frequency (i.e., repetition rate). Since it has already been shown that inductor-diode resonant charging circuits are superior to ence frequency (i.e., repetition rate). Since it has already been shown that inductor-diode resonant charging circuits are superior to most others with regards to transfer efficiencies and range of operating frequencies, it is only logical that this is the type of circuit utilized. Figure 5.4 illustrates the configuration of the charging circuit including the values of some of the components. The power supply is a $20 \mathrm{kv}, 250 \mathrm{ma}, 5 \mathrm{kw}$ unit (Hippotronics Model). The discharge capacitor is a $50 \mathrm{kv}, 15 \mathrm{nf}$ low inductance discharge capacitor rated at 360 pps (pulses per second). Since this capacitor is to be operated at only a fraction of the rated voltage, its operating range with respect to repetition rate may be correspondingly increased. The rated power output of this capacitor is 6,750 watts at $50 \mathrm{kv}$ and 360 pps with a certain percentage of this power appearing as internal heat. At an operating voltage of say $10 \mathrm{kv}$, the repetition may be increased to $(50 / 10)^{1 / 2}(360 \mathrm{pps})=9000$ Hertz without increasing the internal losses of the capacitor. One of the most common modes of failure in this type of capacitor are thermal instabilities caused by overheating of the dielectric. Therefore, at $10 \mathrm{kv}$, the usable repetition rate of this capacitor can be well into the kilohertz range without reducing its useful lifetime. One of the primary design criterion for this charging circuit was that the operating frequency should extend into the kilohertz range. With this in mind, recall that 
This is about 23 percent of the total power available. A high voltage diode chain has also been added to this circuit to allow operation below the resonant frequency. If it was desired to use all of the available power, a much larger inductor would be needed. This would necessarily mean a reduction in the repetition rate possible. The size of this inductor is determined by the maximum voltage and average current needed. This would necessarily mean a reduction in the repetition rate possible. The size of this inductor is determined by the maximum voltage and average current capabilities of the supply.

$$
\begin{aligned}
& \mathrm{z}_{\mathrm{TL}}=(20 \mathrm{kv}) /(.25 \mathrm{~A})=80,000 \mathrm{ohm} \\
& \text { with } \mathrm{Z}_{\mathrm{TL}}=(\mathrm{L} / \mathrm{C})^{1 / 2} \text { with } \mathrm{c}=15 \mathrm{nf}
\end{aligned}
$$$$
\text { Therefore } L=z_{T L}{ }^{2} \mathrm{C}=(80,000 \Omega)^{2}\left(15 \times 10^{-9} \mathrm{nf}\right)=96 \mathrm{hy}
$$

The repetition rate corresponding to this size inductor would be only 265 Hertz. The pulse energy available from the charged capacitor can be determined from the voltage doubling that occurs along with a measurement of the dissipation factor of the charging inductor. The dissipation factor (D.F.) was measured on an impedance bridge* to be 7 percent Referring back to equation (50), the quality factor $Q$ of this circuit can be determined to have a value slightly greater than 11 . Using the measured value of the dissipation factor, the maximum charging voltage on the capacitor can be calculated by consideration of

*ESI Impedance Bridge Model 252 
the energy flow from the supply to the capacitor.

$$
\begin{aligned}
& \left(V_{s}\right)_{\max }=4562 \text { volts } \\
& \left(E_{c}\right)_{\text {theoretical }}=2 \mathrm{C}\left(\mathrm{V}_{\mathrm{s}}\right)_{\max }{ }^{2}=1 / 2(\mathrm{C})\left(\mathrm{V}_{\mathrm{C}}\right)^{2} \max \\
& \left(\mathrm{E}_{\mathrm{c}}\right)_{\text {practical }}=\left(1-\mathrm{D}_{\bullet} \mathrm{F}_{\bullet}\right)\left(\mathrm{E}_{\mathrm{c}}\right) \text { theoretical }
\end{aligned}
$$

Solving for $\left(V_{c}\right)_{\max }$

$$
\begin{aligned}
& \left(V_{c}\right)_{\max }=2\left(V_{s}\right)_{\max }[1-D . F \cdot]^{1 / 2} \\
& \left(V_{c}\right)_{\max }=(2)(4562 \mathrm{v})(1-.07)^{1 / 2}=8799 \text { volts }
\end{aligned}
$$

The maximum energy available from the pulse charging network will then be

$$
\begin{aligned}
& E_{c}=1 / 2 C V^{2}=(1 / 2)\left(15 \times 10^{-9} \mathrm{nf}\right)(8799 V)^{2} \\
& E_{c}=.58 \text { joules }
\end{aligned}
$$

This value of $1 / 2$ joule is only half of the stored energy necessary to realize 1 joule on the output end of the transmission lines. This situation was born out of the need to select an all around power supply for the laboratory and still operate in the kilohertz range. This naturally resulted in a sacrifice in pulse energy on the ouput. If the repetition rate is reduced, then the output pulse energy can be increased up to almost 12 joules per shot.* Thus an extended range of output pulse energies is available by varying the size of the charging inductor and the corresponding repetition rate for a given size discharge capacitor.

\footnotetext{
*This assumes that on $1 y 90$ percent of the energy appearing at the discharge capacitor will be avallable at the load.
} 


\section{EXPERIMENTATION}

\section{Testing Overview}

The testing phases of the present research effort consisted of several parts. The first part revolved around the selection and testing of the liquid dielectrics considered most suitable for incorporating into a liquid filled transmission line (LTL). The two primary candidates were water and glycerine. The most important electrical properties and energy storage capabilities of these two liquids have already been given in Table 1. Referring back to that table, both liquids appear to have suitable energy storage capabilities with water having the ability to deliver a longer pulse for a given transmission line length. Glycerine on the other hand, with its higher dielectric strength, can deliver more power to the load, again for a certain size transmission line. Since each one of these dielectrics possessed certain characteristics favorable to their use, both were tested to determine whether or not there were any operational differences between them. A Marx-Bank was first used to pulse charge a single liquid transmission line filled with either glycerine or water. These two dielectrics were tested in order to determine their maximum practical working voltages as well as their healing times under fault conditions. It was established during this series of experiments that glycerine was more electrically stable than water under no flow conditions. This was evidenced by the fact that water failed to achieve the expected holdoff voltage of $15 \mathrm{kv} / \mathrm{mm}$. 
Experimentation then turned towards measurements of the LTL's charging times with glycerine as the dielectric. Subsequent experiments were aimed at measurements of the risetimes and rates of rise of the current pulses under varying conditions of output inductance and impedance. These latter experiments shed considerable light on the transition region between a lumped parameter and distributed parameter network. The final product of all the above experiments was the gaining of an in-depth understanding of the operating characteristics of LTLs.

During the experiments involved with understanding the characteristics of the transmission lines there was a parallel development of fast pulse charging circuits used to energize the LTLs. The first circuit, a Marx-Bank while fast was deemed far too inefficient because it resistively draws its energy from the power supply. Therefore, this circuit was later replaced with resonant charging network that increased the total overall power transfer efficiency by almost a factor of 10. Final testing was undertaken with this latter system.

\section{Marx Bank Testing}

The first pulse charging circuit constructed was a $34 \mathrm{kv}, 53$ nf two-stage Marx-Bank. The capacitors used in the Marx-Bank are 50 kv, 15 nf capacitors from Sangamo. Figure 6.1 shows the entire Bank which consisted of two stages of seven capacitors per stage. It was necessary to parallel this number of capacitors in order to increase the output capacitance of the Marx-Bank (53 nf) to a value high enough so as to prevent significant voltage droop from occurring during 
charging of the transmission lines. The bank was resistively charged as shown in Figure 5.2. Erection of the Marx-Bank occurred via a standard air-filled spark gap shown in Figure 6.2. One of the electrodes was threaded and moveable, enabling the gap spacing to be varied thereby changing its breakdown voltage and the output voltage of the Marx-Bank. Operating characteristics of the spark gap and supply limited this range to from several kv to almost $35 \mathrm{kv}$. A typical open circuited output voltage waveform of the Marx-Bank is shown in Figures 6.3 through 6.4 .

The output voltage has a 10-90\% risetime of about 23 nsec (Figure 6.4). The speed of the Bank was therefore deemed fast enough to pulse charge the TLs in the time frames of interest (i.e. less than several Usecs). The actual charging of the TLs was accomplished by way of an isolating gap (Figure 6.5) set to be statically triggered on the erection peak of the Marx-Bank. This gap's static breakdown voltage was also variable in the same manner as the Marx-Bank gap. 


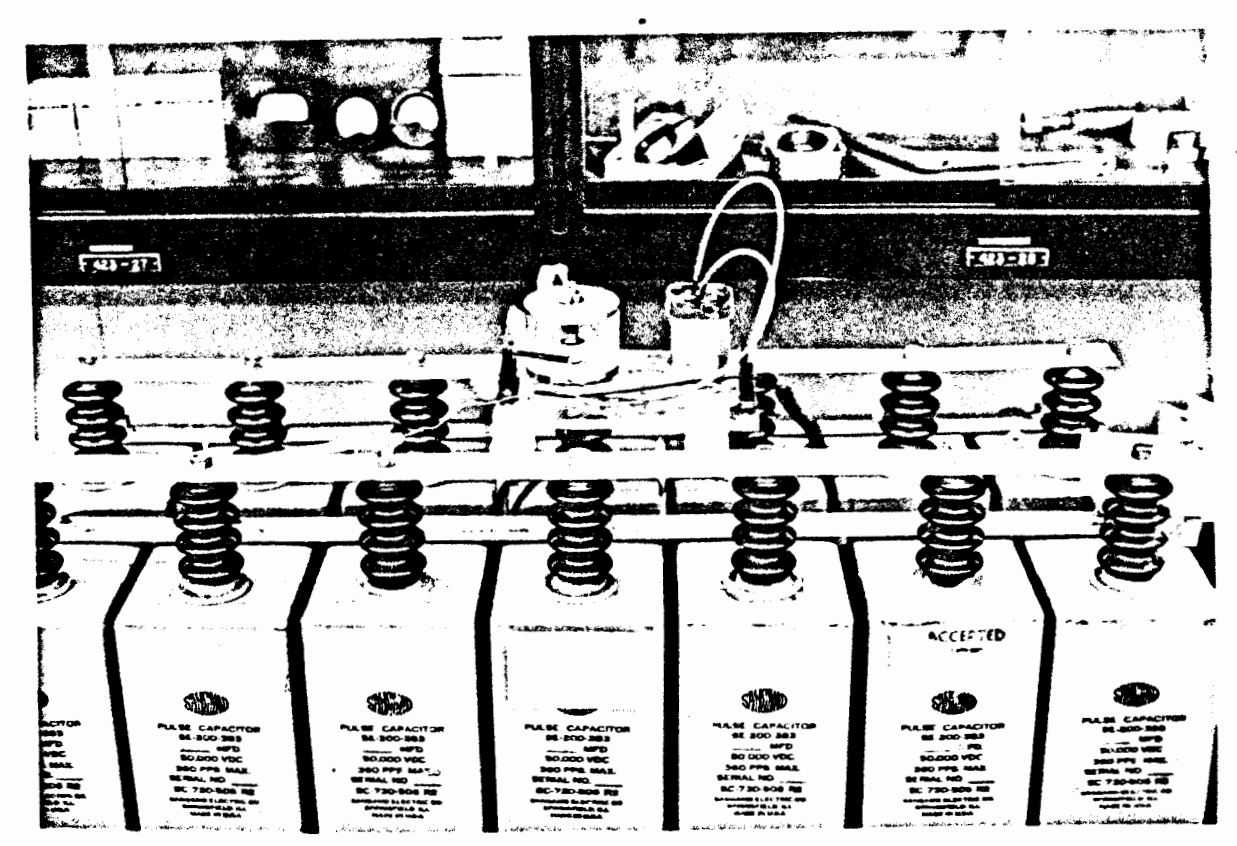

Fig. 6.1. High Voltage Marx-Bank and Transmission Lines

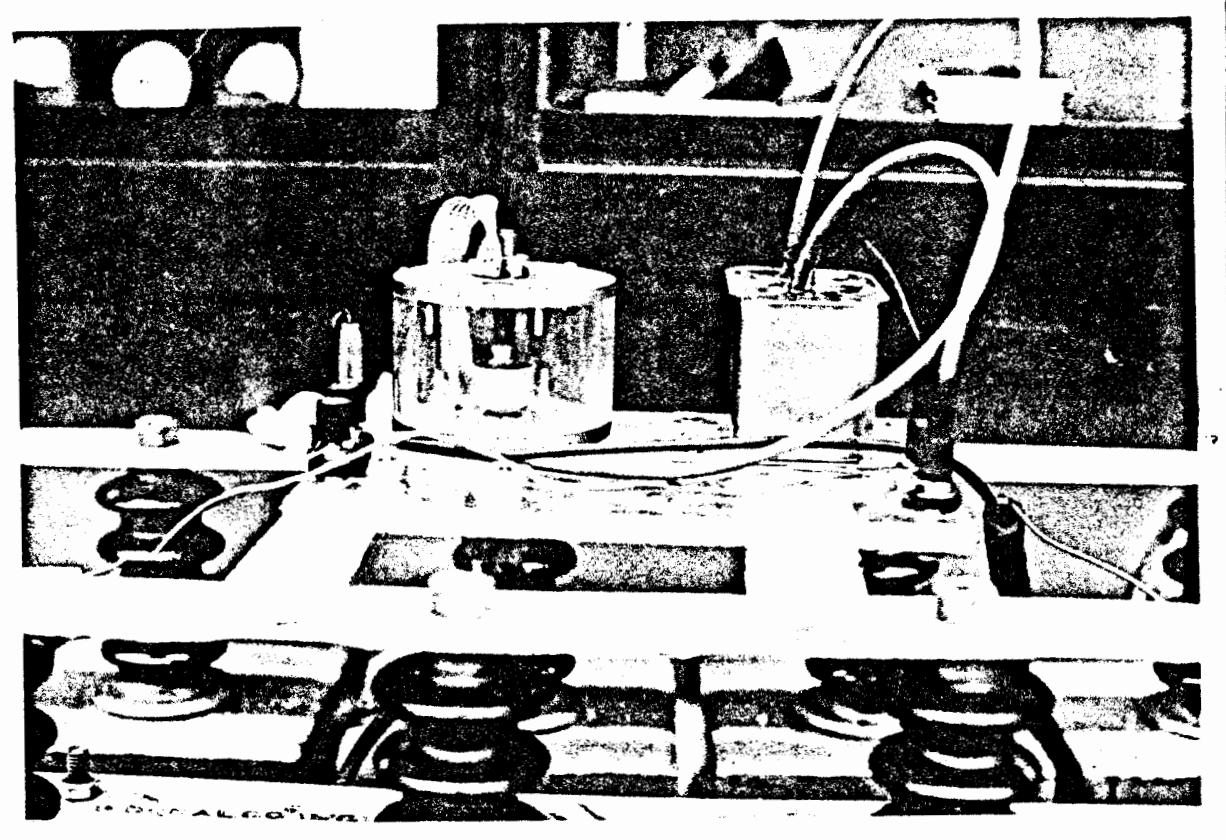

Fig. 6.2. Marx-Bank erection spark gap 
Water was selected first for testing because it was available in a very pure form.* The basic procedure was to prepare the TL structure by thoroughly cleaning the interior with alcohol, enter the water as quickly as possible and then immediately begin testing. The quickness with which each of these latter two tasks was accomplished was deemed necessary in order to minimize the diffusion of ion products and contaminants into the high field regions of the TLs. The ion products and contaminants were assumed to be forming at the mild steel-water interface by the dissolving action of the water. Initially, tests at a $1 \mathrm{~mm}$ electrode separation were very negative. Supposedly the maximum practical working stresses on a negative electrode in water should be about $15 \mathrm{kv} / \mathrm{mm}$. The first tests were at 14 $\mathrm{kv} / \mathrm{mm}$ and breakdown through the TLs occurred on every charging cycle. The TLs were then drained and inspected for damage. The internal discharges had caused the appearance of small pits on the line structures. It was encouraging that these pits were not localized near the edges or ends of the 1ines. Both of these areas had been radiused to prevent field enhancement and premature breakdown. Nor were these pits localized at any certain point on the lines but were distributed rather evenly over the midsection of the line. From this evidence it was concluded that even though the expected field strengths were not reached, the electric field region of the TL structure was of a fairly uniform quality and the breakdowns occurring were statistically

*Distilled in a two-stage all quartz condensing unit. 


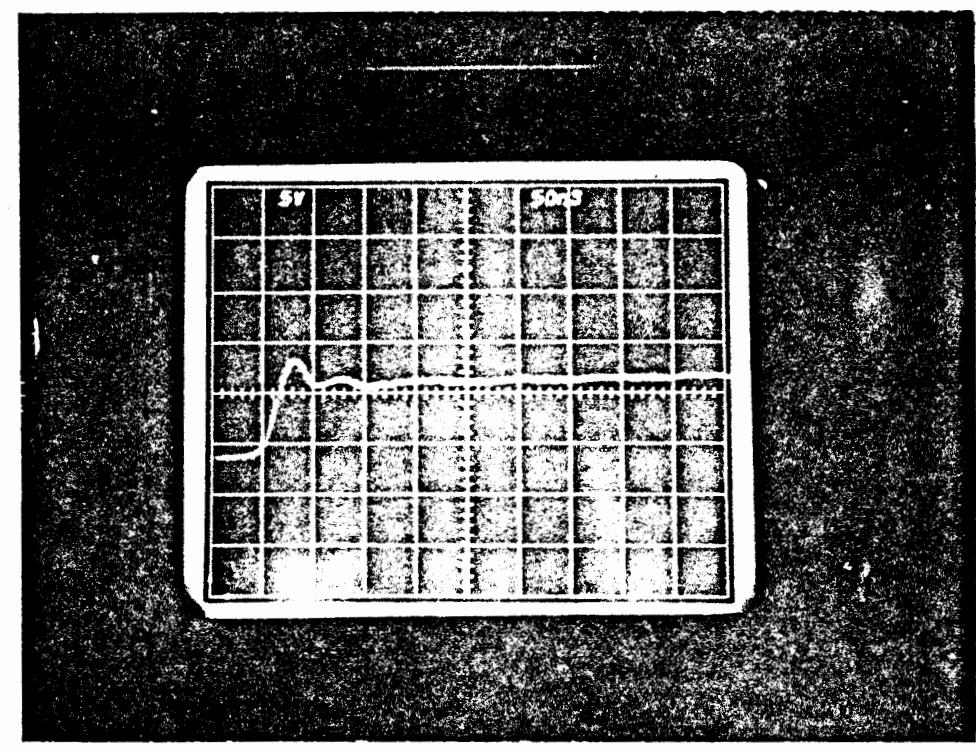

Fig. 6.3. Marx-Bank output voltage waveform

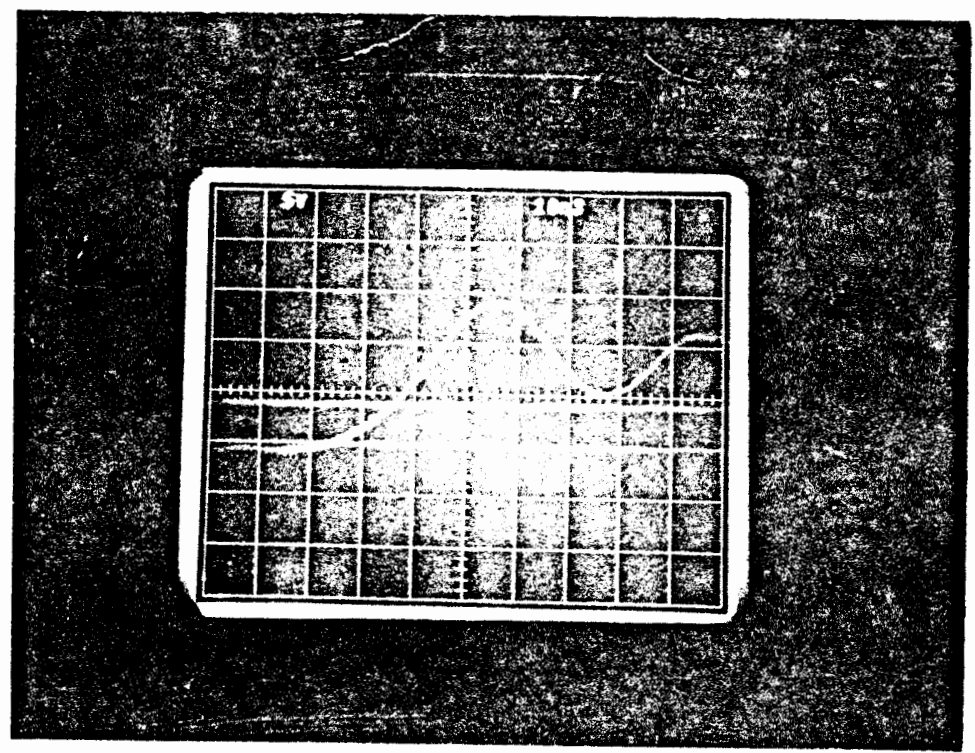

Fig. 6.4. Marx-Bank output voltage waveform (22 nsec risetime, 10-90\%) 
located. This simply means that the breakdowns were occurring at those instances in time at which the right conditions just happen to exist. Subsequent field strength testing of water occurred by lowering the voltage in $2 \mathrm{kv}$ increments from the original $14 \mathrm{kv}$ test voltage. In each of these tests only one TL was used with a constant spacing of 1 mm. After each test the system was drained, dismantled and painstakingly cleaned. All of the discharge pits from the previous experiment were smoothed off with very fine emery cloth. The test voltage steadily decreased downward from $12 \mathrm{kv}$ to $10 \mathrm{kv}$ to $8 \mathrm{kv}$, and finally, at 6,000 volts, hold-off occurred and sparking took place on the output gaps of the TLs. The output gaps consisted of opposing 1/8-inch diameter stainless steel pin electrodes of variable spacing. Testing proceeded around the $6 \mathrm{kv}$ hold-off voltage and it was determined experimentally that the maximum stress obtainable with water under static or no flow conditions was no more than $7 \mathrm{kv} / \mathrm{mm}$. This reduced the storage capabilities of water by more than a factor of four. The reason for this poor performance by water is probably related to the ionization products being produced through contact with the mildsteel box structure in which the three stainless steel TLs are housed. From conversations with several knowledgeable individuals as we11 as documentation found in several articles it was concluded that without continual flow and deionization, water would only be a substandard energy storage medium. $(18,23)$ For the lack of a suitable deionizing and filtering system, it was decided to abandon the use of water and turn to glycerine instead. 
Reagent grade glycerine was obtained from the Chemistry Department and used to replace water as the TL medium. The results of the change were very positive. At two different spacings, $1 / 2$ and 1 $\mathrm{mm}$, breakdown strengths of 21 and $23 \mathrm{kv}$ per mm were achieved with 200 to $300 \mathrm{nsec}$ charging times. This is an average of $22 \mathrm{kv} / \mathrm{mm}$ and not too much different from the $25 \mathrm{kv} / \mathrm{mm}$ referenced in Table 1 . All subsequent experimentation in this research program was with glycerine and no other liquid dielectrics were tested.

\section{Self-Healing Times}

One of the most important properties of liquid dielectrics is their ability to heal themselves once an internal discharge through them has occurred. In this respect it was decided to visually observe breakdown and then reapply system voltage with a varying time lag between breakdown and voltage application. This same experiment had been run earlier with water with the following results. The selfhealing time of water was found to be fairly fast. After breakdown had occurred, the voltage was reapplied at a reduced level by varying the input voltage from the power supply. It was found that most of the time the system voltage could not be reapplied fast enough to cause premature or anomalous breakdown. No visual change in the medium was observed. Glycerine on the other hand reacted somewhat different. Every time an internal breakdown occurred there appeared a black cloud of ionization products along the trail of the discharge. If the voltage was reapplied too fast, anomalous breakdown occurred usually 
very close to the region of the last breakdown. It took anywhere from 10-30 seconds for glycerine to heal depending upon the severity of the breakdown and the number of times that it occurred at any one location. Visually it could be seen that the black ionization products would slowly rise to the surface of the liquid. It might be that once the chain of ionization products from one electrode to the other has been sufficiently broken, the hold-off voltage will reappear.

These experiments showed that water clearly holds an edge over the more viscous glycerine regarding healing times. If glycerine is to be used in a highly stressed, high repetition rate mode there should be some sort of fault indicator and relay network used to interrupt the flow of energy for a time period sufficiently long enough for the dielectric to heal itself. In this respect it would also be very desirable to circulate the fluid and filter any contaminants from it. With water this can be accomplished rather easily, while with glycerine it presents a problem because of its higher viscosity.

\section{TL Charging Voltage Waveforms}

Since efficient use of the energy storage capabilities of liquids require the systems to be pulse-charged, it would be instructive to measure the actual charging times involved in the present system and determine the relative magnitude of the internal losses caused by bleed-off during the charging cycle. During the charging cycle a TL acts as a capacitor with a time constant of $\boldsymbol{\tau}_{c}$. The waveforms in Figures 6.6 through 6.10 are representative of the charging waveforms on the TL when pulse charged from the Marx Bank. The 
voltage risetime from zero to $95 \%$ full charge $\left(5 \tau_{c}\right)$ varies from 100 nsec in Figure 6.6 to 250 usec in Figure 6.9. Comparison of the waveforms shows that there is a distinct difference between them with respect to the waveshape at the time of breakdown. It should be remembered that the discharge is operating in a self-breakdown mode. A tesla coil arc was applied to the lower electrode to provide the discharge gap with some initial ionization which reduced the static breakdown voltage for a given gap separation. The optimum breakdown mode occurred when the peak charging voltage was just enough to cause breakdown. If the voltage is insufficient to cause breakdown, no discharge will take place and the TL's entire supply of stored charge will bleed-off internally, resulting in an efficiency factor of zero for that particular pulse. If on the other hand the peak charging voltage is much greater than that required for breakdown, discharge of the TLs will take place on the rising portion of the wavefront. Since the risetime of the charging voltage on the TLs is independent of the final peak voltage,* premature breakdown will occur with a concomitant reduction in risetime. That is exactly what occurs in the above photographs. In Figure 6.6 the input charging voltage is clearly much more than is required to initiate conduction. Close inspection of Figure 6.6 will reveal that after initial breakdown takes place at Point A, the voltage levels off from A and B and then proceeds to fall toward zero. This leveling off is the result of an interaction between the charging and discharging voltage pulses. The discharge pulse can

\footnotetext{
* See Equation 40
} 


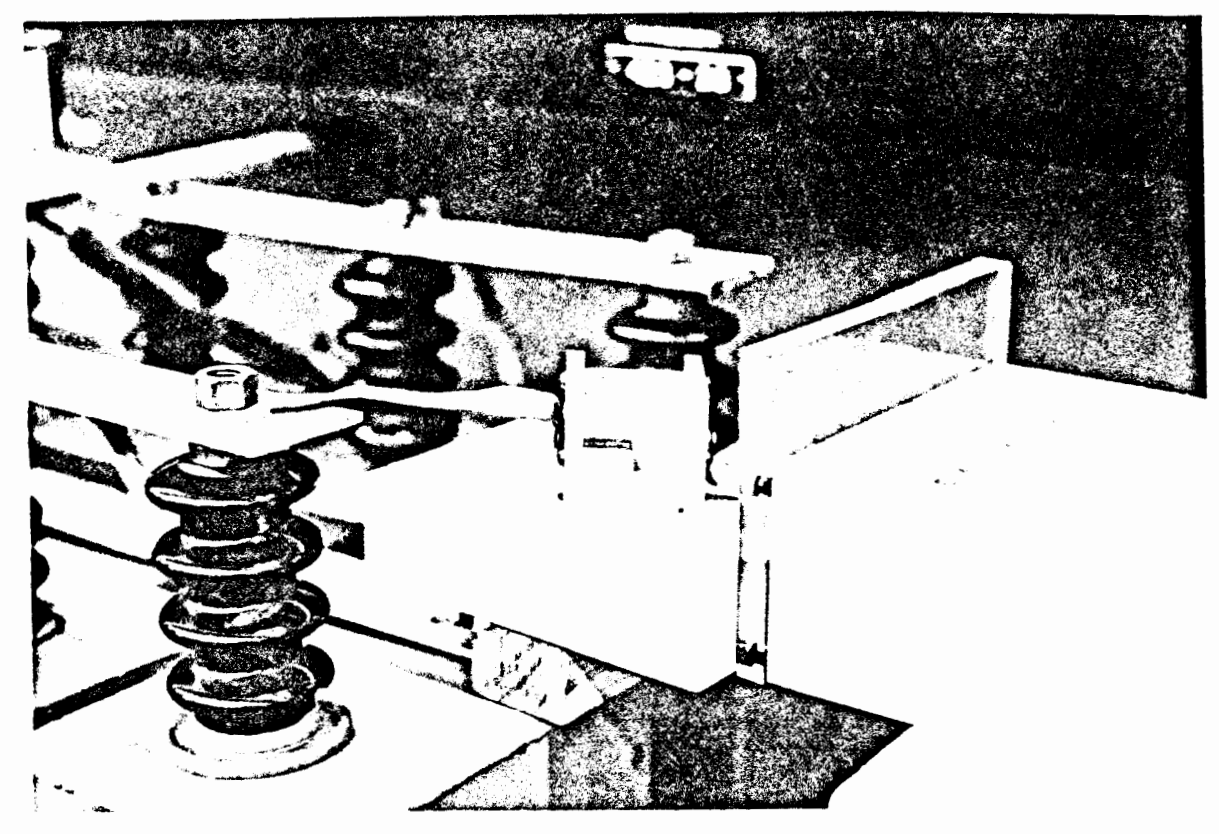

Fig. 6.5. Marx-Bank isolating spark gap

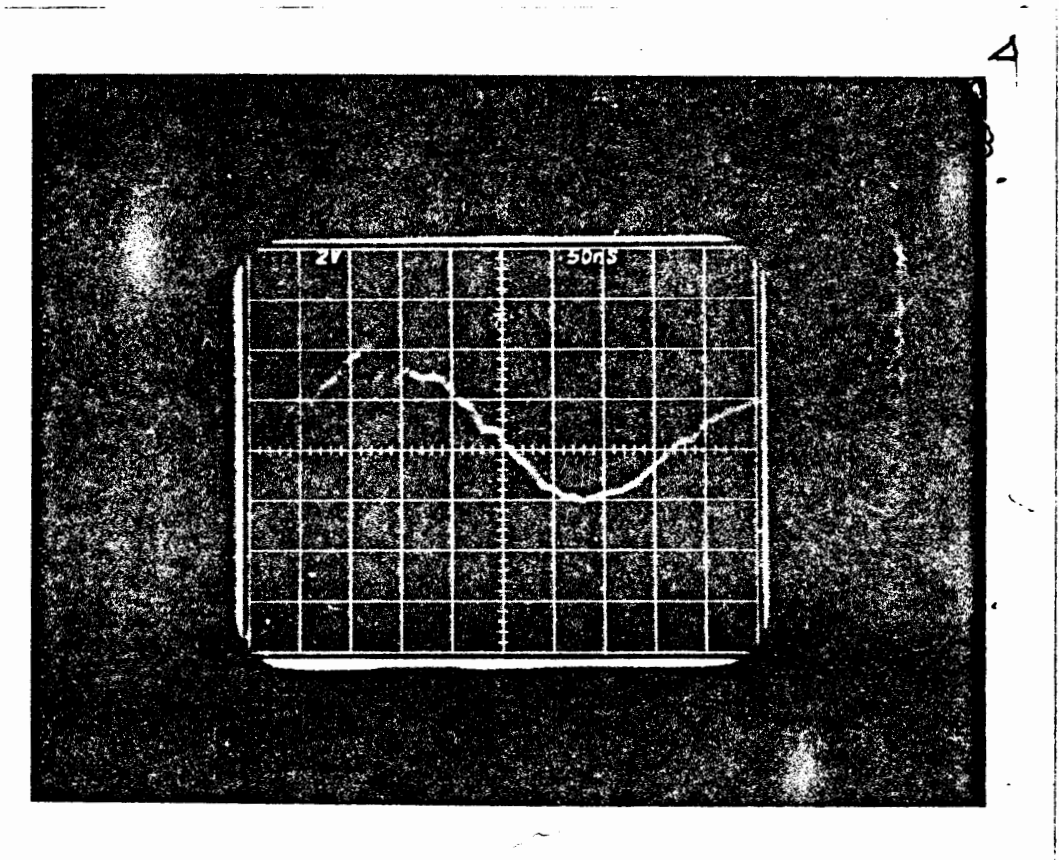

Fig. 6.6. TL charging voltage waveform (Anomalous breakdown) 


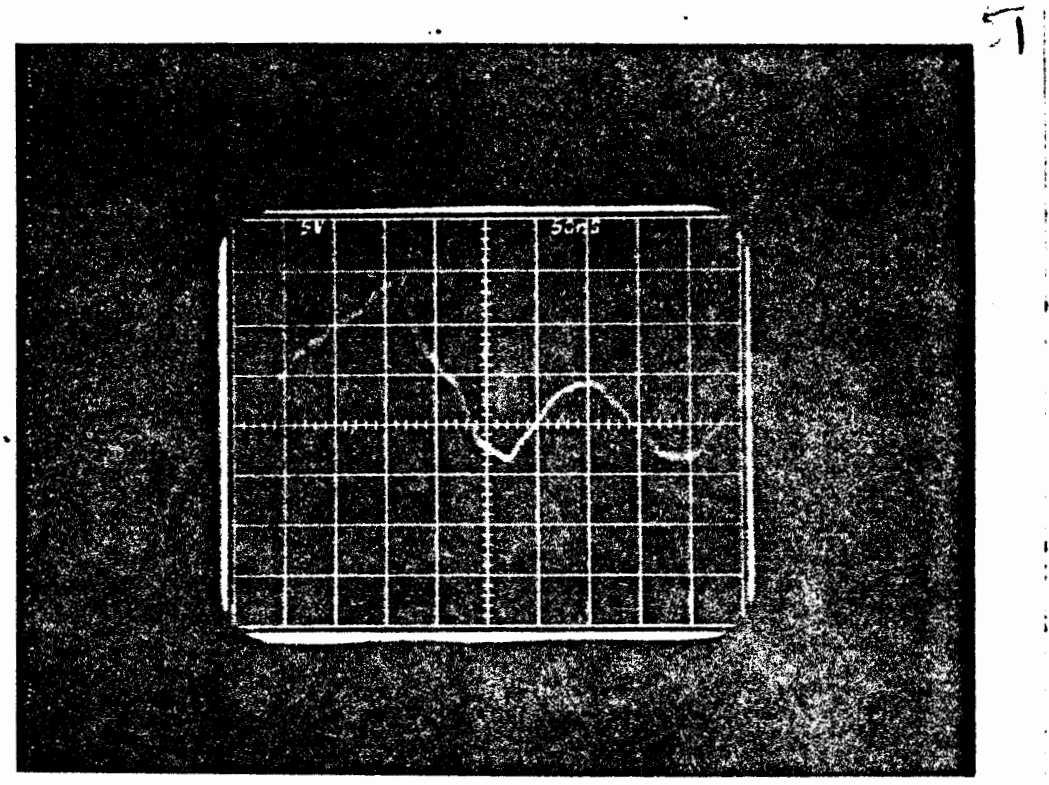

Fig. 6.7. TL charging voltage waveform
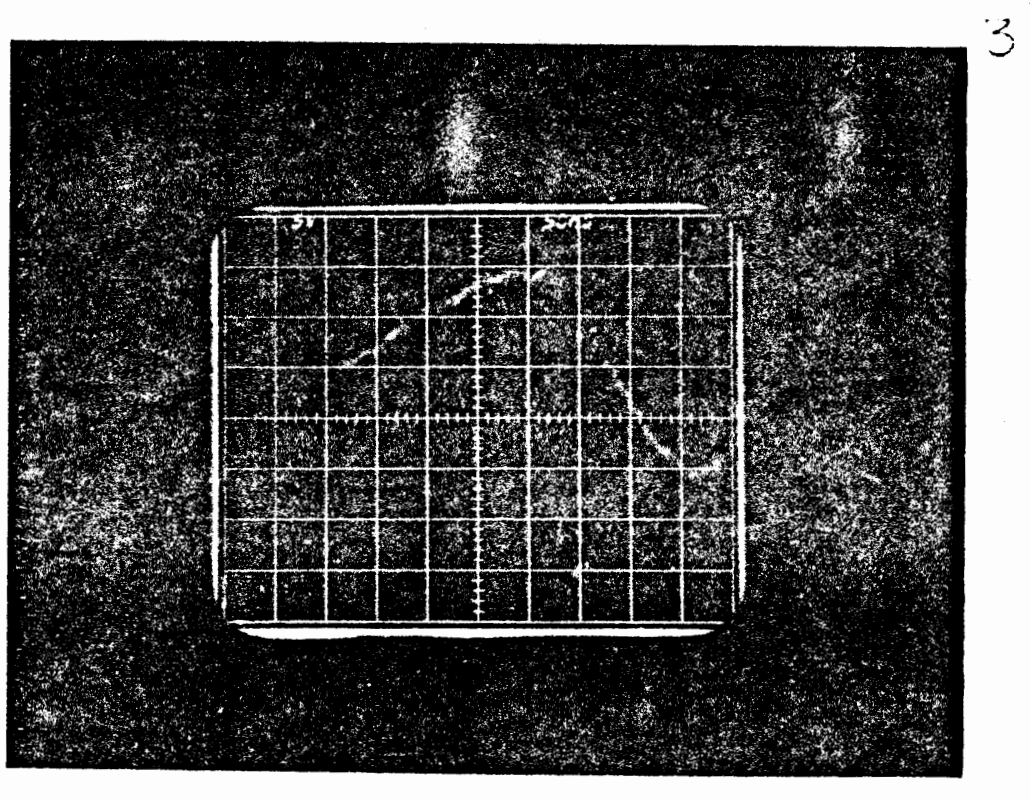

Fig. 6.8. TL charging voltage waveform 


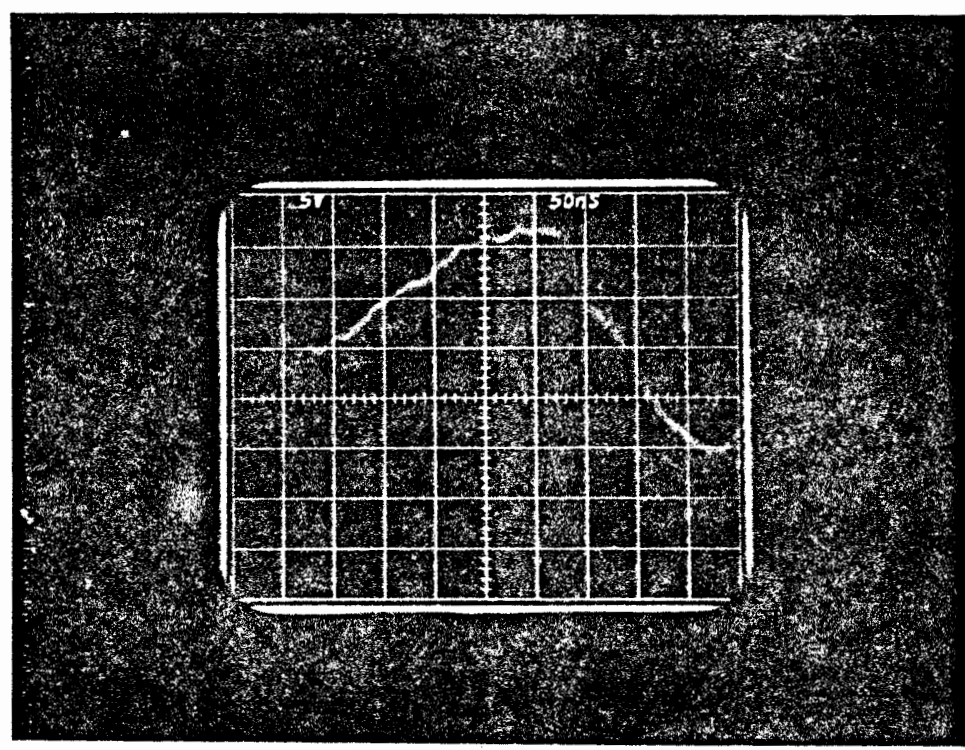

Fig. 6.9. TL charging voltage waveform

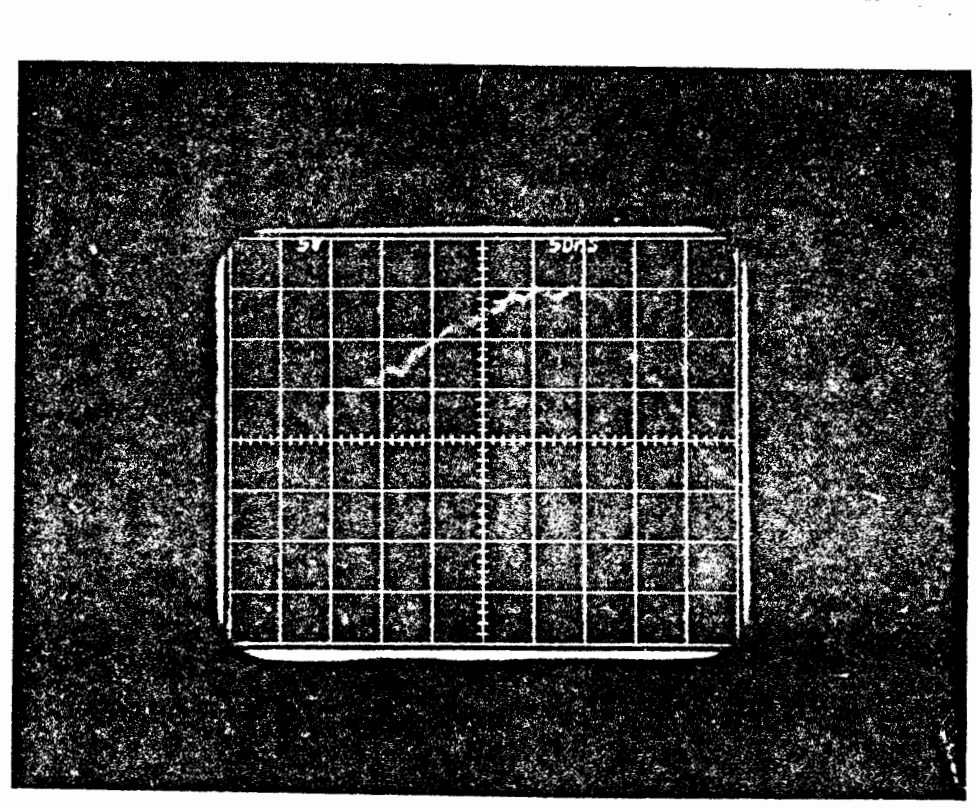

Fig. 6.10. TL charging voltage waveform 
be modeled as a negative voltage wave appearing at the instant of breakdown and traveling in the opposite direction as the charging pulse. If the system voltage would have peaked out at the time of discharge the resulting waveform would show only a gradual decline toward zero potential and no step would have occurred (see Figure 6.9-6.11). But, if the voltage is still rising at the time of breakdown the negative voltage wave will encounter a rapidly changing positive wave and application of the principle of superposition will show that an intermediate voltage step can appear. As the gap separation is increased which corresponds to an increase in static breakdown strength, the waveforms start to assume the proper shape (see Figures 6.8 and 6.9 ). Notice how breakdown occurs on the rounded, slowly changing portion of the waveform. This indicates that peaking is occurring at the time of discharge. Pulse charging is inefficient enough without also operating in a premature breakdown mode. This type of operation can easily be avoided by proper adjustment of the input voltage. For any given electrode spacing, the input voltage should be slowly raised until a consistent sparking action results. This will ensure optimum use of the available energy in the pulse charging circuit.

At this point it should be stressed that even though overvoltaging is a common technique for causing uniform discharge breakdown along a laser channel, it is definitely not energy efficient in pulse charging circuits. The function of uniform discharge triggering has to be separated from the charging circuit in order to achieve both goals 
of efficient energy use and uniform discharge triggering • On the average, the charging time for the TLs is about $250 \mathrm{msec}$. This is approximately equivalent to a $1 \mathrm{MHz}$ charging signal. From Von Hipple,(23) the loss tangent ( $\mathcal{d}$ ) for glycerine at $1 \mathrm{MHz}$ is approximately $300 \times 10^{+4}$. Charging losses are therefore about $2 \%$ Since the TLs do not always discharge exactly on the voltage peak, but are sometimes delayed, there are additional losses which occur by internal bleedoff. These latter losses may be determined by consideration of the RC decay constant for a DC signal. This constant has previously been given by $m \in$, where $m$ is the dielectric resistivity and $\epsilon$ the permittivity. The resistivity of glycerine is given by reference (24) to be $15.6 \mathrm{~m} \Omega-\mathrm{cm}$ and $\in$ the permittivity is given by the same reference to be $3.9 \times 10^{10} \mathrm{f} / \mathrm{m}$.

Therefore $\quad R C=m \epsilon=(15.6 \mathrm{~m} \Omega-\mathrm{cm})\left(3.9 \times 10^{-8} \mathrm{f} / \mathrm{cm}\right)=60.8 \mathrm{usec}$

The magnitude of the internal energy loss will be equal to

$$
\text { Loss }=[1-(V(t) / V p k)]
$$

with $\left.V(t)=V_{p k}\left[1-e^{t / R C}\right)\right]$ where $t$ becomes the time lag interval. From Figure 6.10 this is determined to be no more than 100 nsec. Substituting this value into the time interval, the internal losses due to bleed-off are found to be much less than $1 \%$. Therefore in this case, the major portion of the losses occur during the initial charging cycle and time lag decay losses are minimal.

In all the aforementioned cases, the Marx-Bank was the primary source for the pulse charging circuit. Later measurements with 
the resonant charging scheme yielded somewhat better results. The busbar impedance between the discharge capacitor and the TLs was significantly reduced by decreasing the conductor separation, (Figure 6.11), thereby increasing the peak charging current of the circuit. This naturally led to a decrease in the voltage risetime on the TLs from $250 \mathrm{nsec}$ to approximately $180 \mathrm{nsec}$ (see the lower trace in Figure 6.20). This increased rate of charging will naturally reduce bleedoff losses, but it will also increase the skin losses associated with the higher discharge currents and charging frequency. From an efficiency standpoint, the shorter 180 nsec charging times are probably not much better than the longer $250 \mathrm{nsec}$ ones for these reasons.

\section{Capacitive Matching}

The major difference in the two pulse charging circuits utilized is the output capacitance of each. The lower output capacitance of the resonant scheme (15 nf) as compared to the Marx network (53 nf) will enable it to be more closely matched to that of the transmission lines. It should he remembered that exact capacitive matching between source and load (TLs) will maximize the power transfer efficiency. Figures 6.12 and 6.13 show the source voltage of the capacitor as a function of time in the resonant case. Notice that the peak charging voltage is about $4.4 \mathrm{kv}$ before discharge and about $2.2 \mathrm{kv}$ after it has discharged and energized the TLs. These values of initial and final capacitor voltages reflect the fact that resonant transfer of the energy from the capacitor to the storage line is not occurring, 


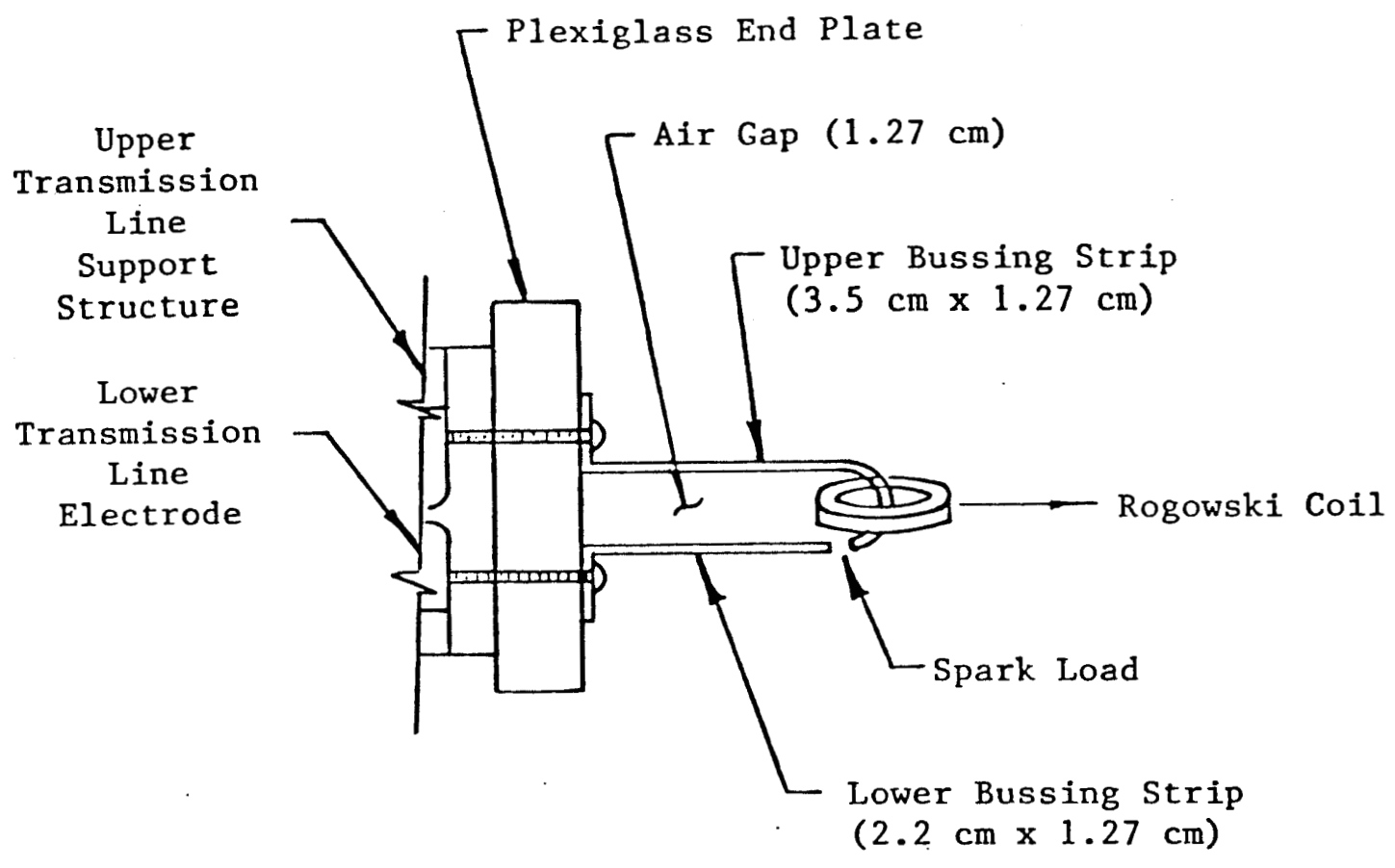

Fig. 6.11. New busbar configuration

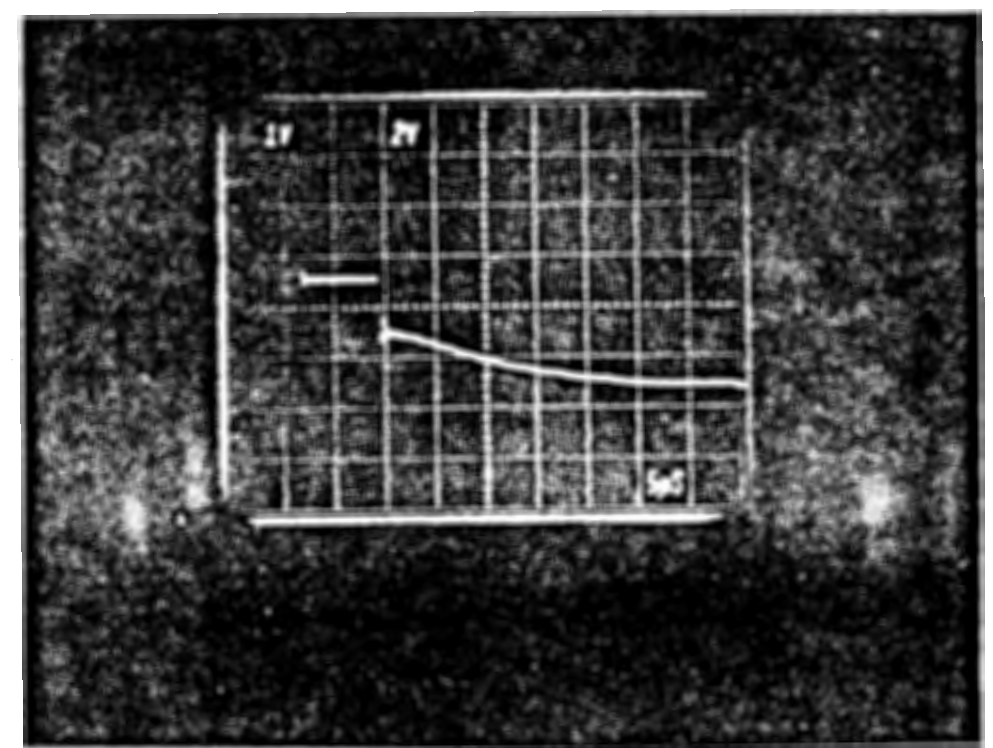

Fig. 6.12. Capacitor voltage, before and after breakdown 
otherwise the capacitor voltage would be reduced to zero. Analysis of the waveforms for the capacitive matching experiments (Figures 6.18 and 6.19) show that at the charging frequency of $1.4 \mathrm{MHz}$ the quality factor of the circuit is less than 3. From this value of the quality factor it can be determined from equation (50) that the transfer efficiency should be about 70 percent. This agrees well with the 25 percent of the total amount of initial system energy remaining on the capacitor as determined from the voltage on the capacitor (2.2 kv) after charging of the TLs. Another phenomenom that is apparently occurring at low repetition rates is bleed-off of the capacitor. Notice in Figure 6.12 that the voltage level at the time of discharge is significantly less than just after resonant recharging has occurred. The explanation for this phenomenom probably lies in the quality of the diode chain chosen to prevent this type of bleedoff. It appears that there is insufficient reverse blocking impedance for keeping the capacitor fully charged for more than a fraction of a second. It should be added that at higher repetition rates there is no bleed-off noticeable (Figure 6.14 through 6.15 ). This condition can probably be lived with or corrected by using a better quality diode. Capacitive matching between source and load is very important because it minimizes late and undesirable energy delivery. Figures 6.16 through 6.19 illustrate this behavior.

Figures 6.16 and 6.17 were taken with the Marx-Bank as the pulse charging source. Notice how both figures show the existence of two distinct oscillation frequencies. The higher frequency corresponds to the TL discharge while the one its superimposed on belongs to the 


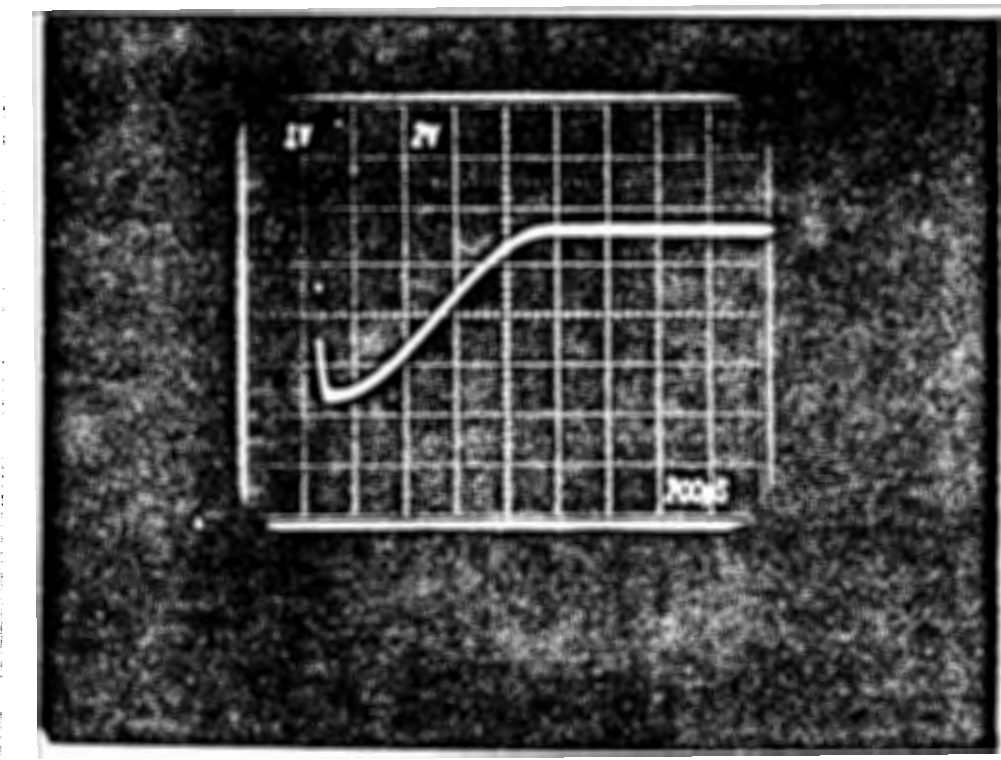

Fig. 6.13. Capacitor voltage, before and after breakdown

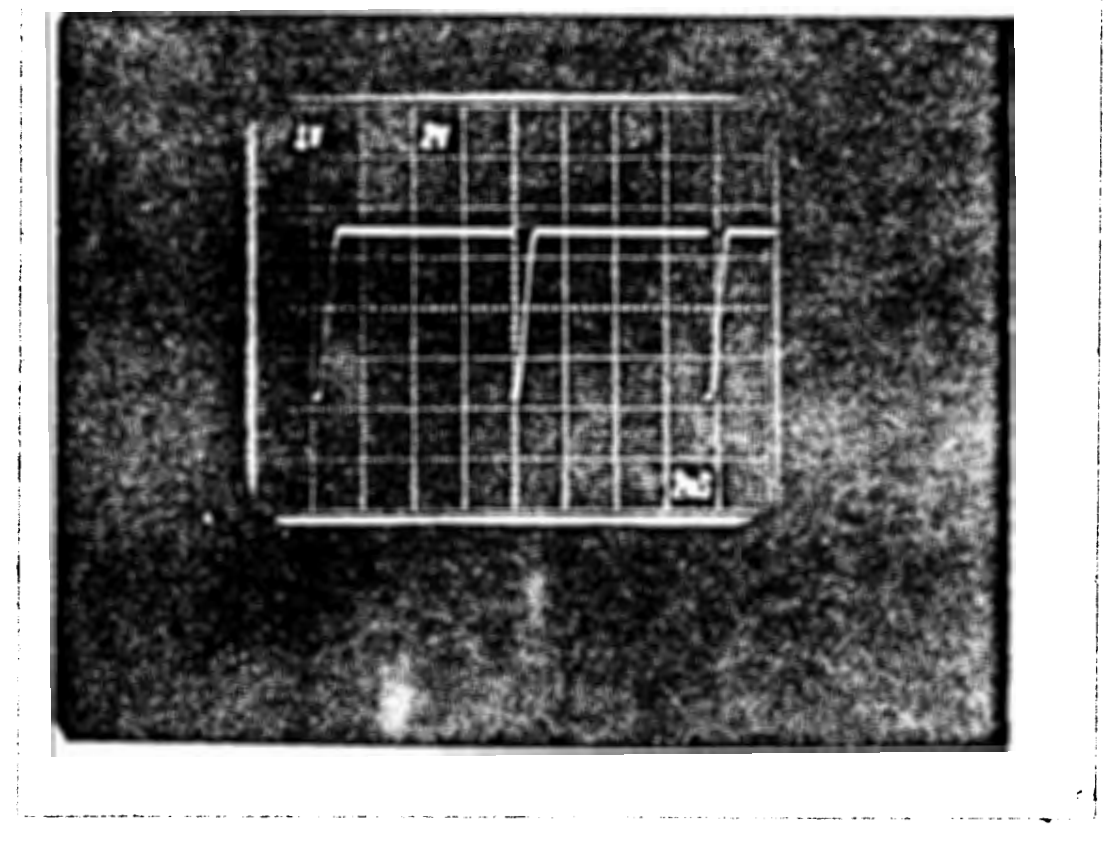

Fig. 6.14. Capacitor voltage (143 pps) 
discharging Marx-Bank. The difference between these two photographs lies in the capacitance of the TL as compared to that of the Marx. Figure 6.18 has a much lower capacitance associated with it than Figure 6.19. It should also be remembered that the impedance of the TL is inversely proportional to its capacitance. Therefore, a lower capacitive TL should also have a higher output impedance which would reduce the first current peak it experiences for a given charging voltage. That is exactly what is borne out in Figures 6.18 and 6.19 These photographs were taken with a Tektronix 7504 single beam oscilloscope and a Physics International model 205 Rogowski coil. The coil was accidentally linked backwards by the discharge loop for these experiments, hence the need to have the voltage inverted on the scope. Later, the same experiment was repeated but with the resonant charging network and a Tektronix dual beam 7704 oscilloscope. The resonant charging scheme with its lower output capacitance allowed it to be exactly matched to the TL, while the dual beam capability allowed observation of both the current and the voltage simultaneously. Notice in Figure 6.20 how the envelope of the TL current and voltage oscillations decay rather uniformly to zero. On the other hand, in Figure 6.21 there is a distinct and appreciable driving force acting past the time at which the TL oscillations have died out. This clearly shows the need to operate pulse charging circuits in capacitive matched modes in order to make the best possible use of the available energy. 


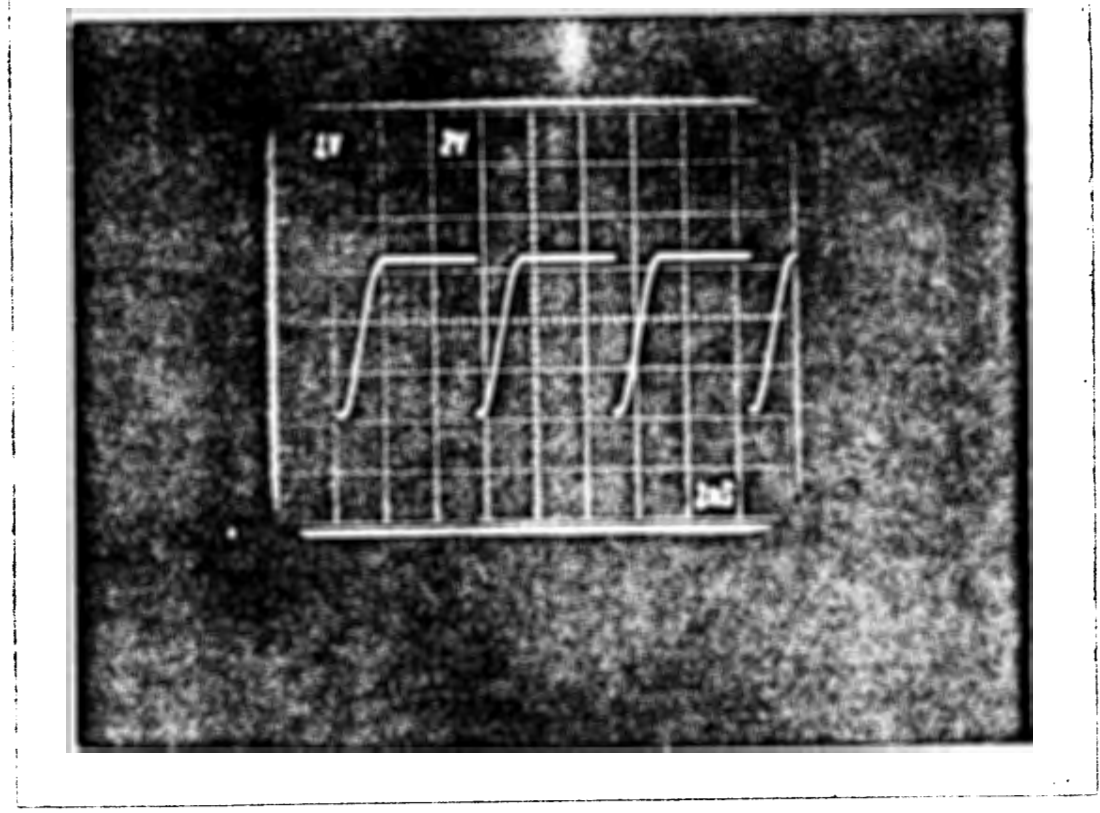

Fig. 6.15. Capacitor voltage (555 pps)

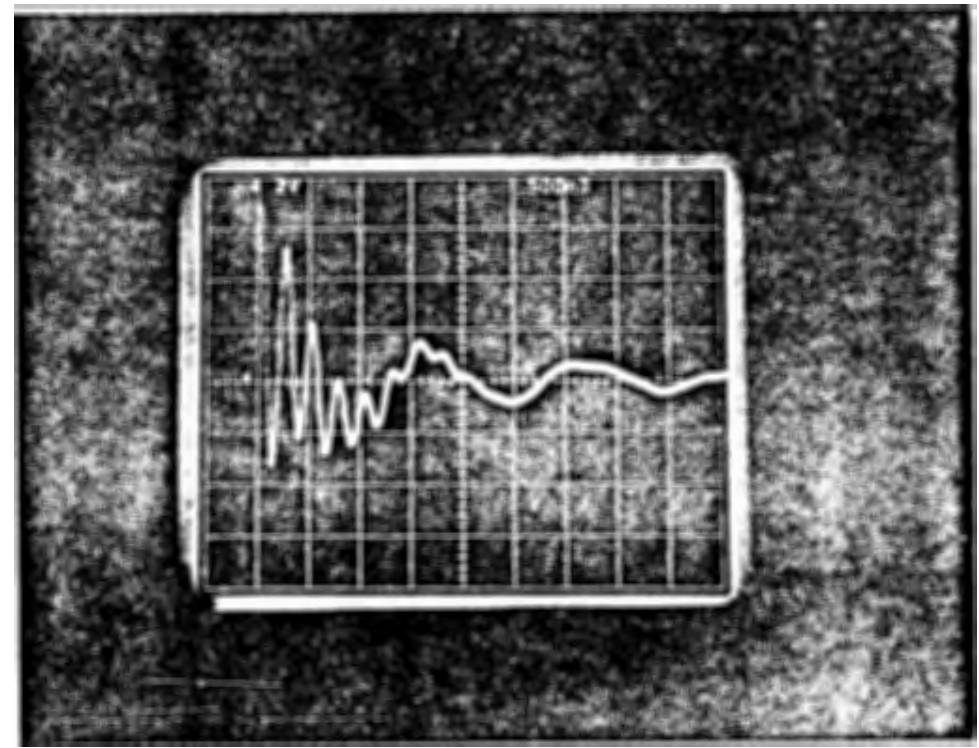

Fig. 6.16. TL current output waveform (source capacitance $=6 \mathrm{TL}$ capacitance) 


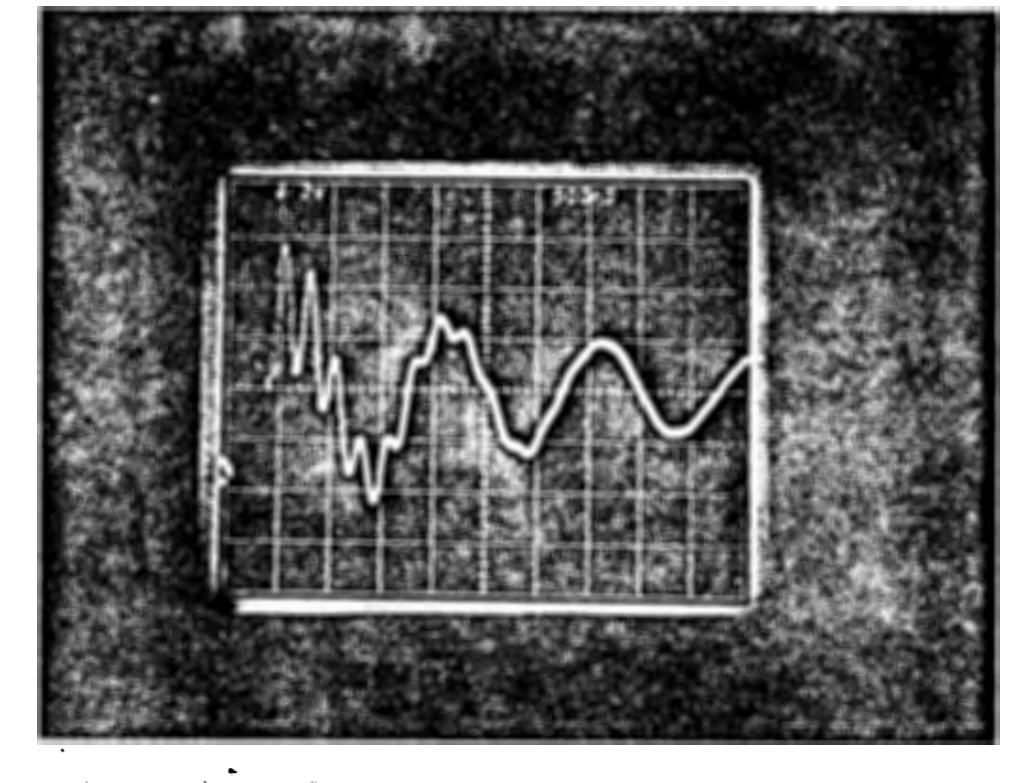

Fig. 6.17. TL current output waveform (source capacitance $=10 \mathrm{TL}$ capacitance)

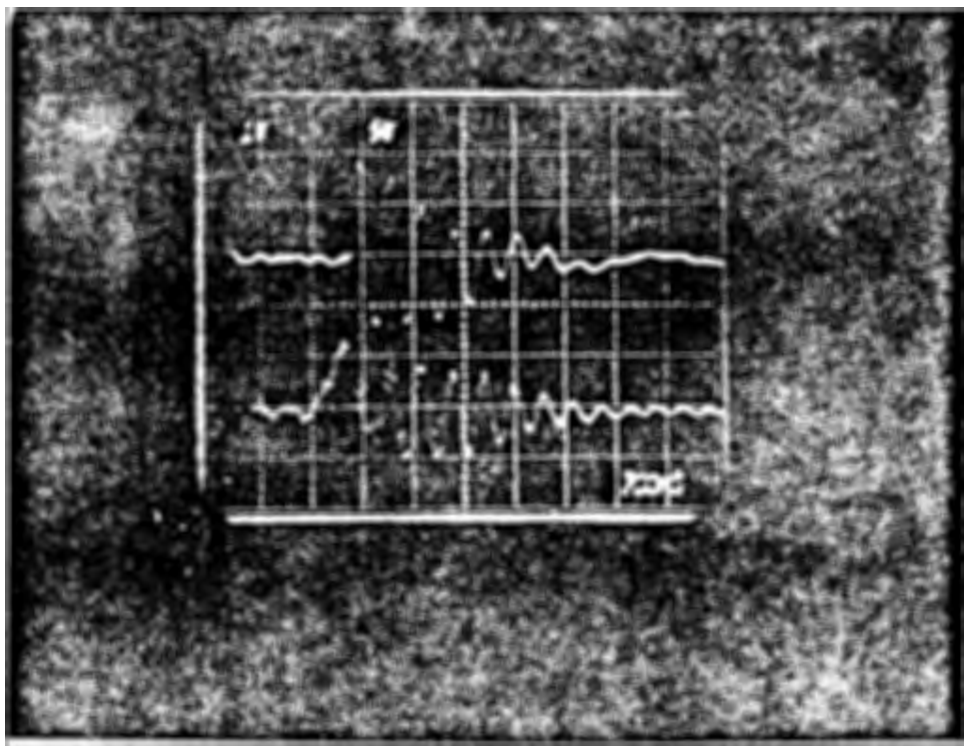

Fig. 6.18. TL current and voltage waveforms (source capacitance = TL capacitance) 


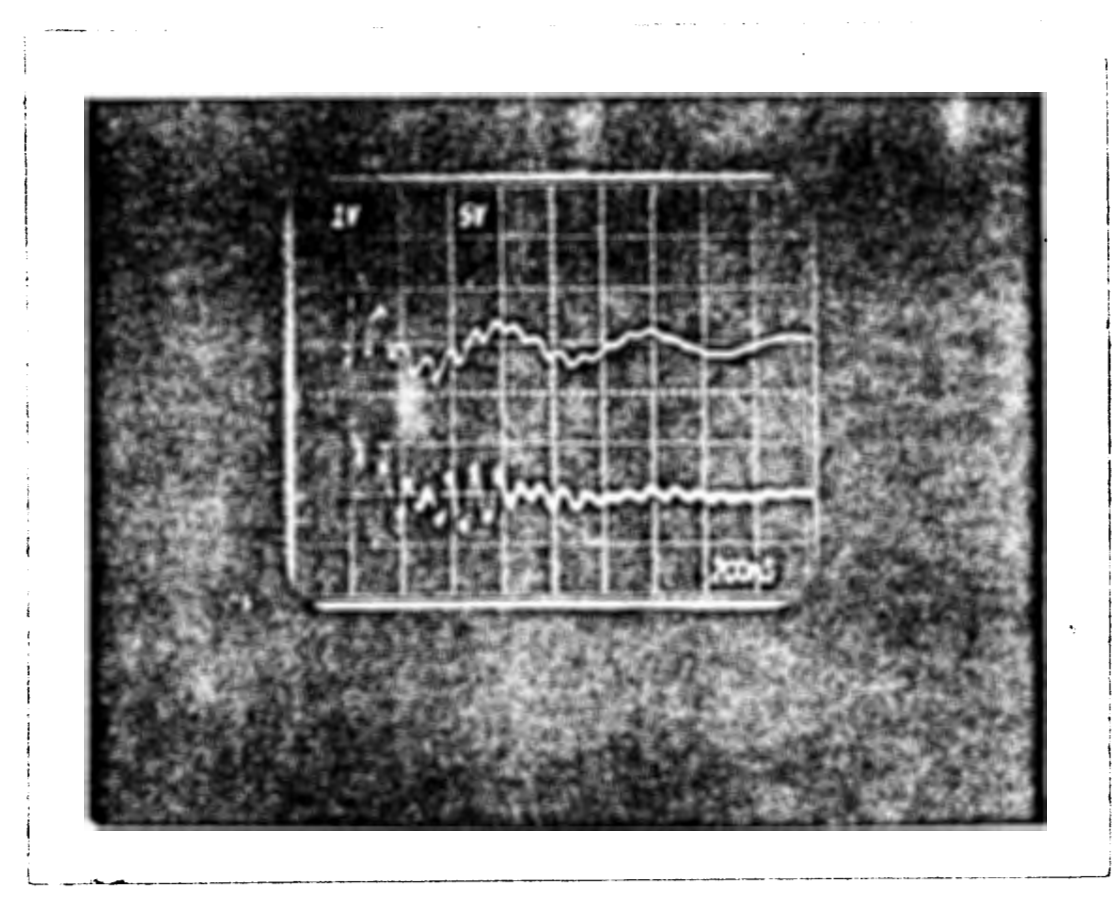

Fig. 6.19. TL current and voltage waveform (source capacitance 〉> TL capacitance)

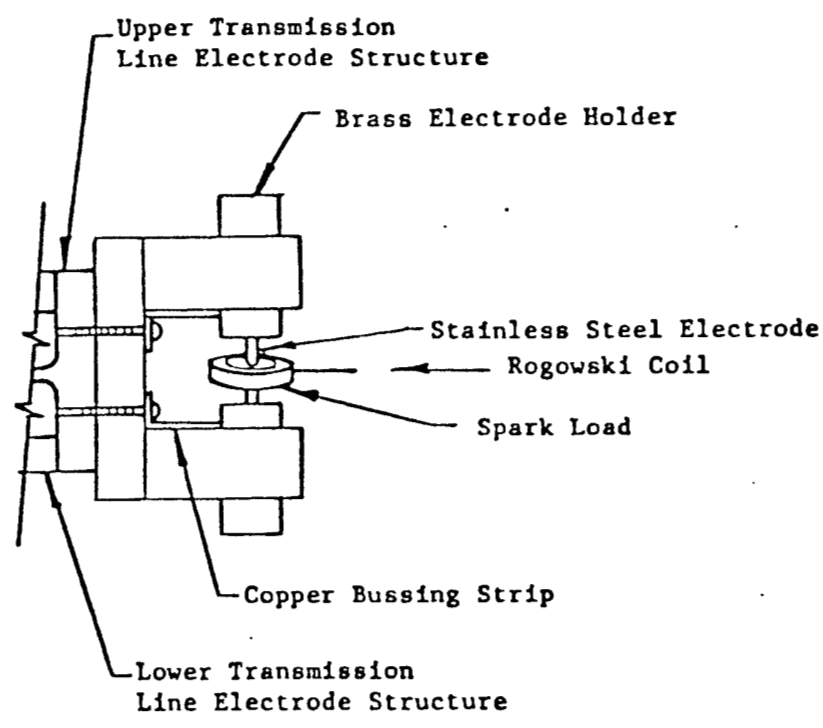

Fig. 6.20. Stainless steel pin electrode configuration 
One of the basic design criterion of the TL discharge circuit was that it generate a rectangular shaped output pulse. To achieve this criterion it is necessary to reduce the pulse risetime to much less than half the one way transit time of the TL (see the section on pulse shaping). Much experimental time and effort was therefore directed toward measurements of current pulse risetimes under varying conditions of output inductance and impedance. The two principal output configurations tested are illustrated in Figures 6.20 and 6.21 . Figure 6.20 is of the first configuration tested. It consisted of a TL output busing network and a stainless steel electrode arrangement originally designed for use in a high intensity spark lamp array. The main geometrical variable in this configuration was the electrode spacing. Changing the electrode spacing varied both the arc inductance and the load impedance. This enabled observation of the effect of these two variables on both the risetime and the impedance matching characteristics between the load and the Tls. Table 2 in Appendix 3 lists the measured and calculated results for the entire series of experiments with certain test series being referenced here for analysis purposes.

The transmission lines were initially set at their designed spacing of $1 \mathrm{~mm}$. and measurements of the charging voltage waveforms and current pulse outputs were made. The voltage was measured with a 6015 Tektronix hi-voltage probe while the discharge current was monitored with the PI Rogowski coil. Figure 6.22 is typical of the current waveforms observed. The results of the first set of experiment (Series 
A) indicated that the current risetimes were too long to show the flat top characteristics of a discharging distributed parameter network. The next series of tests (Series B) were aimed at reducing the inductance of the output configuration by reducing the spark gap spacing of the load. In Reference (6) it was reported that in many instances the arc inductance was a major contributor to the total loop inductance of a discharge circuit. Therefore, by varying the arc length it should be possible to reduce its effect on the total loop inductance of the transmission line's output section. The spark electrode separation distance $\mathrm{S}_{\mathrm{SL}}$ was varied from $1 \mathrm{~mm}$ to $2.88 \mathrm{~mm}$ and finally all the way up to $4.88 \mathrm{~mm}$. Current and voltage measurements were made at each separation. The results are tabulated in Table 2. Figures 6.23 through 6.25 are representative of the photographs taken of the current pulse waveform during this series of experiments.

At first, it was very confusing because no matter what spacing was used all the risetimes were in the neighborhood of 50 nsec. In fact, closer inspection of the data made it even more confusing because one of the shorter risetimes observed was with the largest spacing $(4.88 \mathrm{~mm})$ and one of the longest risetimes with the smallest spacing $(1 \mathrm{~mm})$. The reason for these results was traced to the fact that all the experiments were not correlated with a single input voltage and peak current. In other words, a faster rising or sharper pulse can have a risetime much longer than a slower rising pulse if its peak value is much greater than in the latter case. Therefore, a much 
>

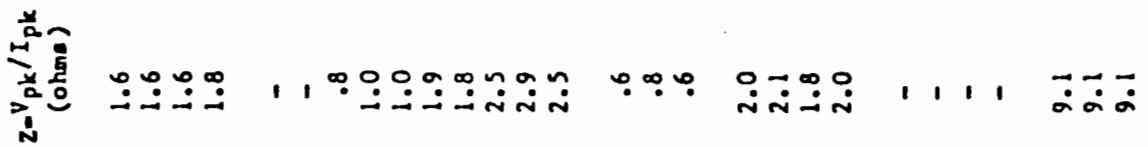

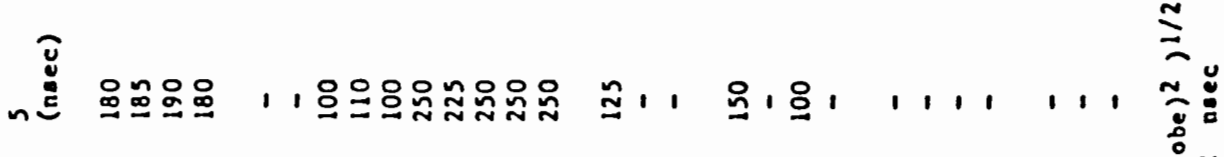

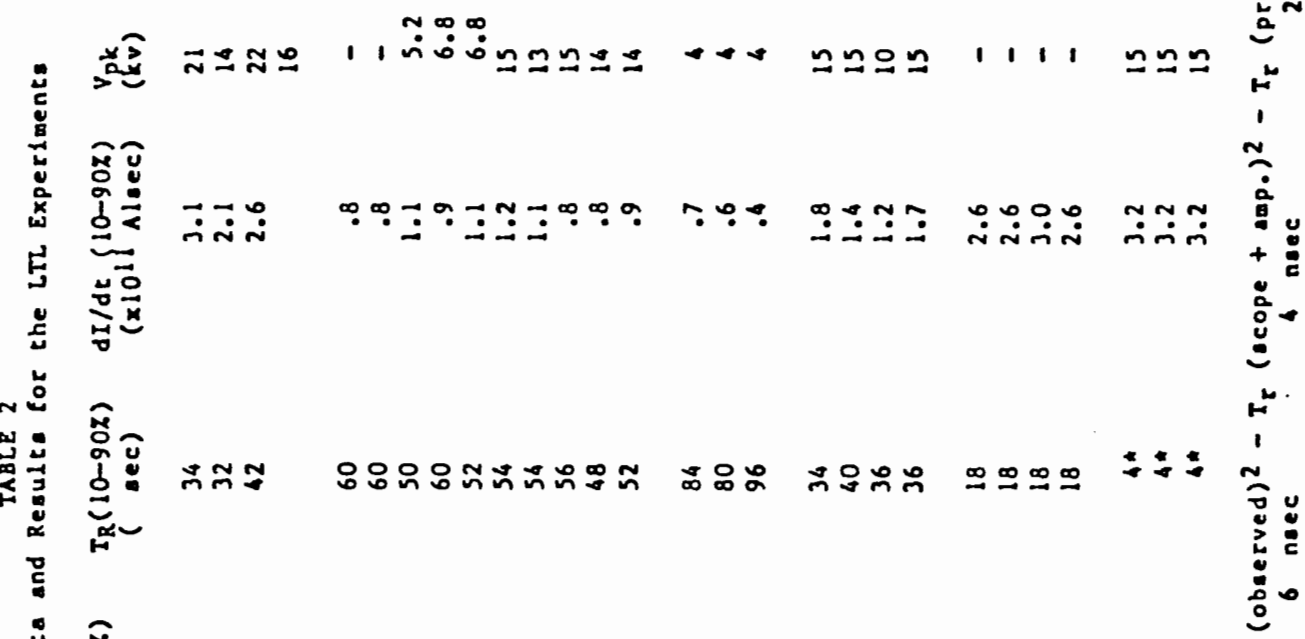

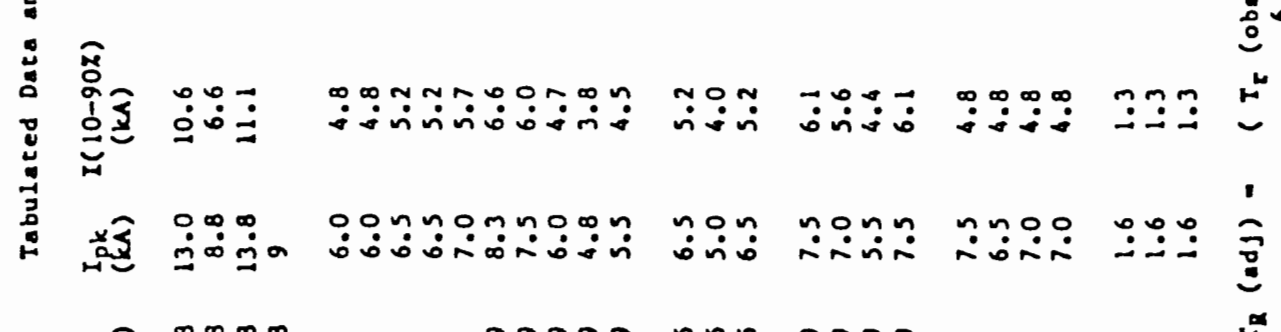

矛

庭

南 ……

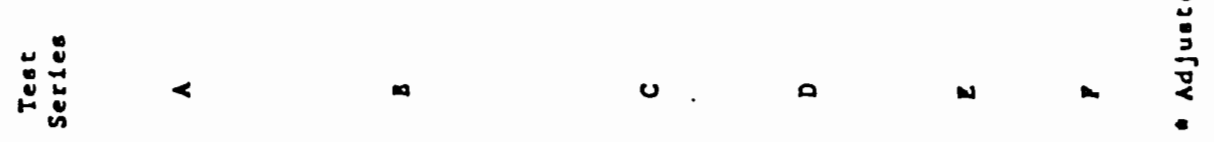




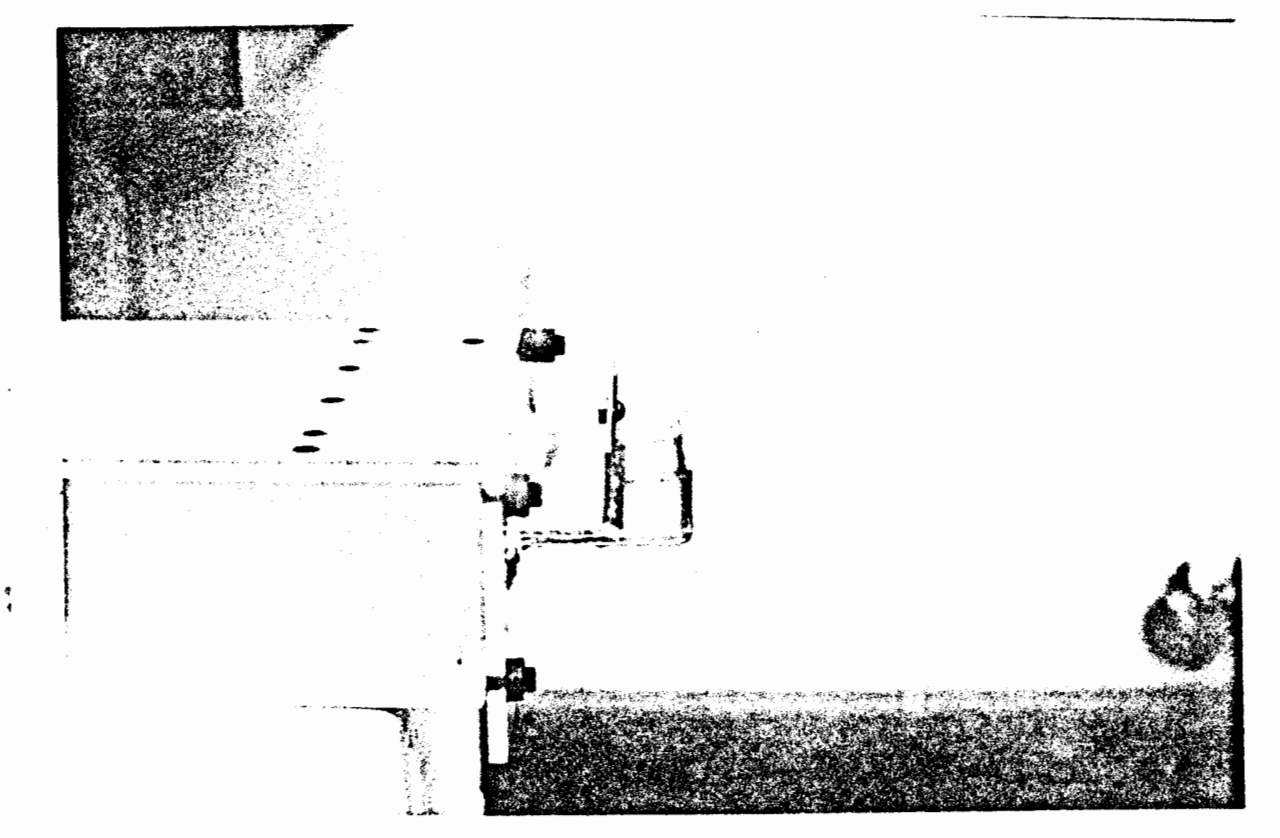

Fig. 6.21. Low inductance output configuration

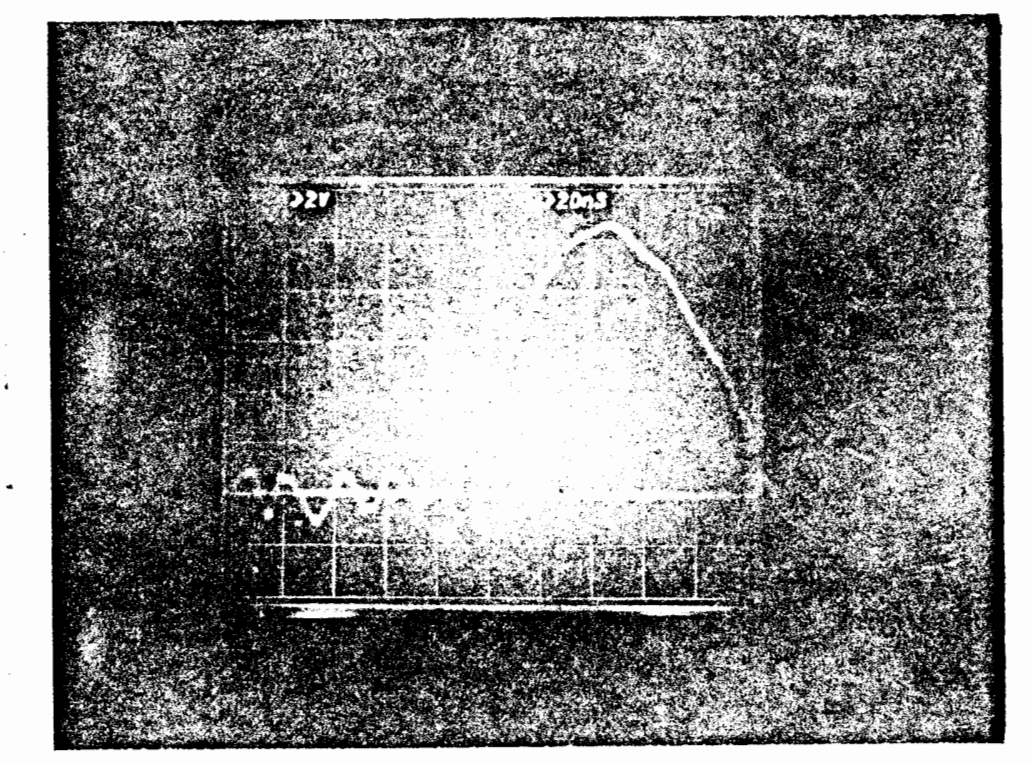

Fig. 6.22. TL current output waveform 


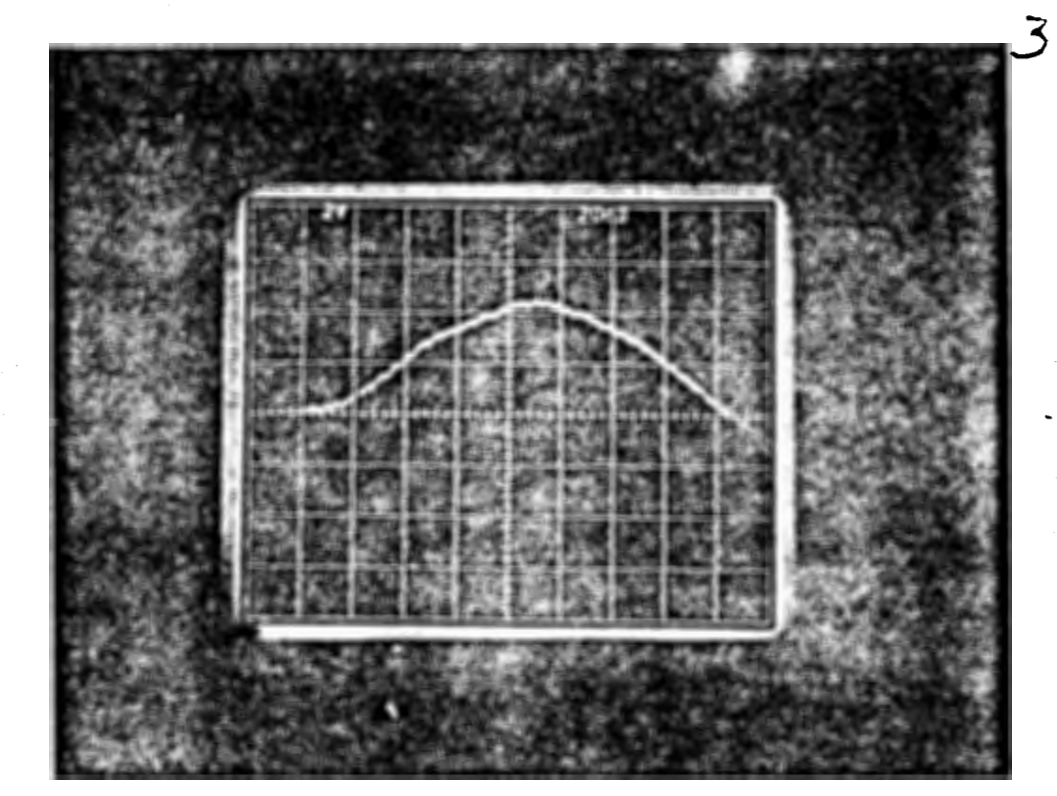

$\therefore$

Fig. 6.23. TL current output waveform

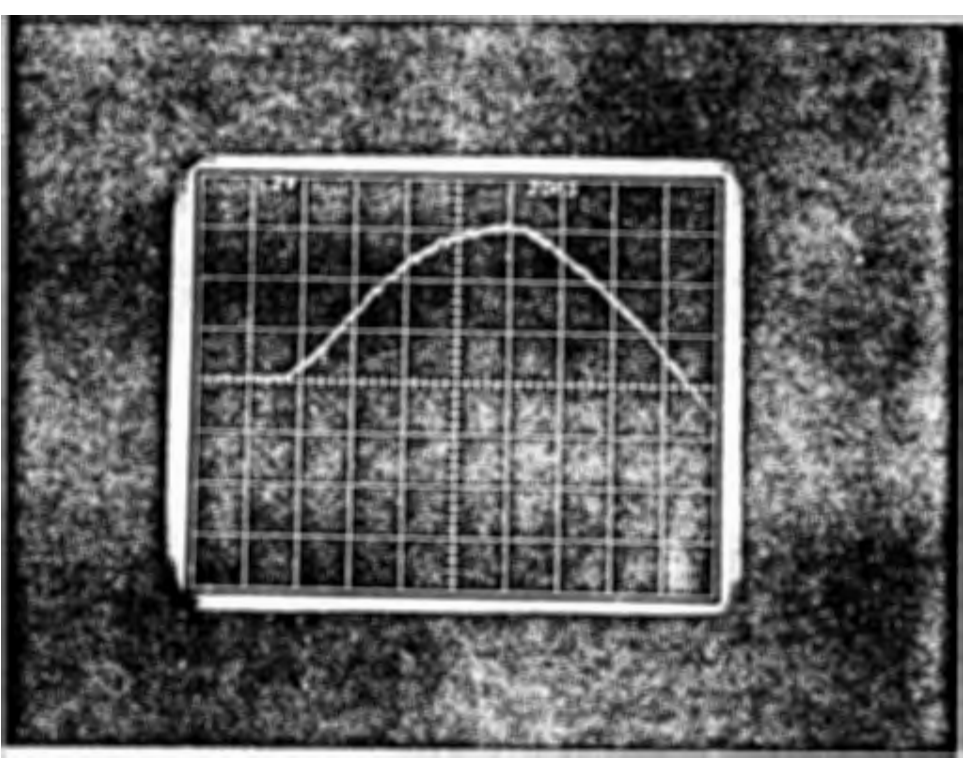

Fig. 6.24. TL current output waveform 
better indicator of what is occurring as the arc inductance varies for a given input voltage would be $d I / d t$, the rate of change of the current on the pulse wavefront. Comparison of the rates of current change in the second set of experiments showed that the anticipated inverse relationship between output inductance and $\mathrm{dI} / \mathrm{dt}$ did take place. Since the values for the $1 \mathrm{~mm}$ electrode spacing experiment were taken at only a $5 \mathrm{kv}$ input voltage as compared to the $7.5 \mathrm{kv}$ used in the other two cases it was necessary to correct these values of $d I / d t$ by a factor of 1.5 (i.e. 7.5/5.0). This correction is necessary in order that any variation in $d I / d t$ be only attributable to a change in output inductance and not output voltage. The results are plotted in Figure 6.26 It can be seen that as the arc length and its corresponding inductance is increased the rate of change of the current on the pulse wavefront goes down. This inverse relationship between output inductance and current rate of change is exactly what should occur.

It was also realized at this time that the reason the risetimes were not changing was because the peak values of the current pulses, I pk, were changing at approximately the same rate as $\mathrm{dI} / \mathrm{dt}$ was changing. The peak value of the current pulse is given by equation (22) to be

$$
\mathrm{I}_{\mathrm{pk}}=\mathrm{v}_{\mathrm{pk}} /\left(\mathrm{Z}_{\mathrm{TL}}+\mathrm{R}_{\mathrm{L}}\right)
$$

For any given $T L$ spacing, $Z_{T L}$ is constant and for a given input voltage $\mathrm{V}_{\mathrm{pk}}$ the only remaining variable is the load impedance $\mathrm{R}_{\mathrm{L}}$. The load impedance consists of a series combination of two components, $\mathrm{R}_{\mathrm{L}}$ and $\mathrm{L}$. 


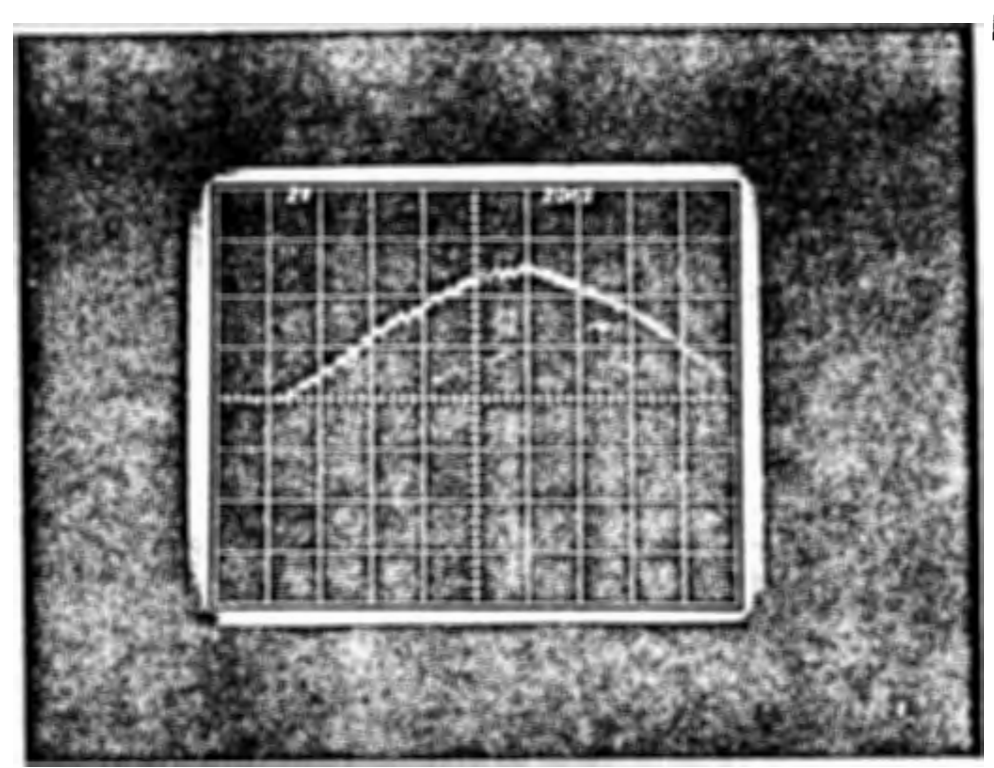

F1g. 6.25. TL current output waveform

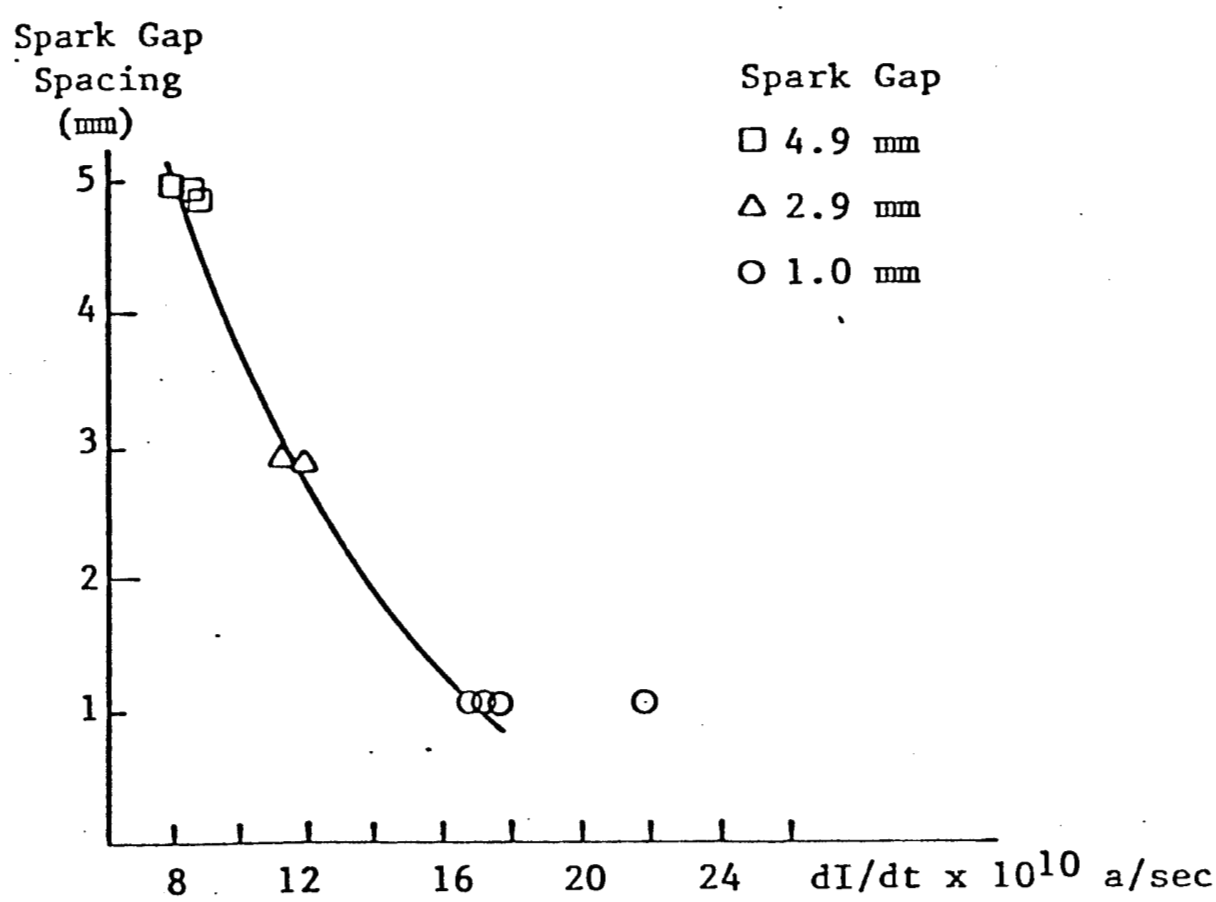

Fig. 6.26. Spark electrode spacing vs $\mathrm{dI} / \mathrm{dt}$ 
$R_{L}$ is the resistive component of the output configuration. $R_{L}$ includes skin resistances and all ohmic resistances in the spark itself. The inductive part of the load impedance $L$ is composed of all the series inductances in the output configuration which includes all busing inductances and the inductance of the spark itself. As the spark electrode spacing is increased, both $R_{L}$ and $L$ will increase also. In the Series B experiments, the increase in the inductive component has reduced the $d I / d t$ on the pulse wavefront, but it also in combination with the resistive part has limited the magnitude of the first current peak. These two effects occurring simultaneously were the reasons that the pulse risetimes were not changing. It is also the reason why increased damping of the TL discharge was not observed. Damping is dependent only upon the resistive component of the discharge loop and not the total impedance. While increasing the length of the discharge did increase the total load impedance, evidently the increased ohmic resistance of the spark was not of sufficient magnitude to manifest itself as an increase in the damping factor of the circuit. Figure 6.27 is a plot of the total load impedance versus the spark electrode spacing. The calculated load impedance values for the $1 \mathrm{~mm}$ spacing using equation (22) had to be adjusted because the peak voltage observed on the traces was much below the output of the Marx. If the observed value for the voltage was used to calculate the total ouput impedance, its value fell below the natural impedance of the TL which is theoretically impossible. It was determined that in cases of premature breakdown, the peak discharge current would depend upon the 


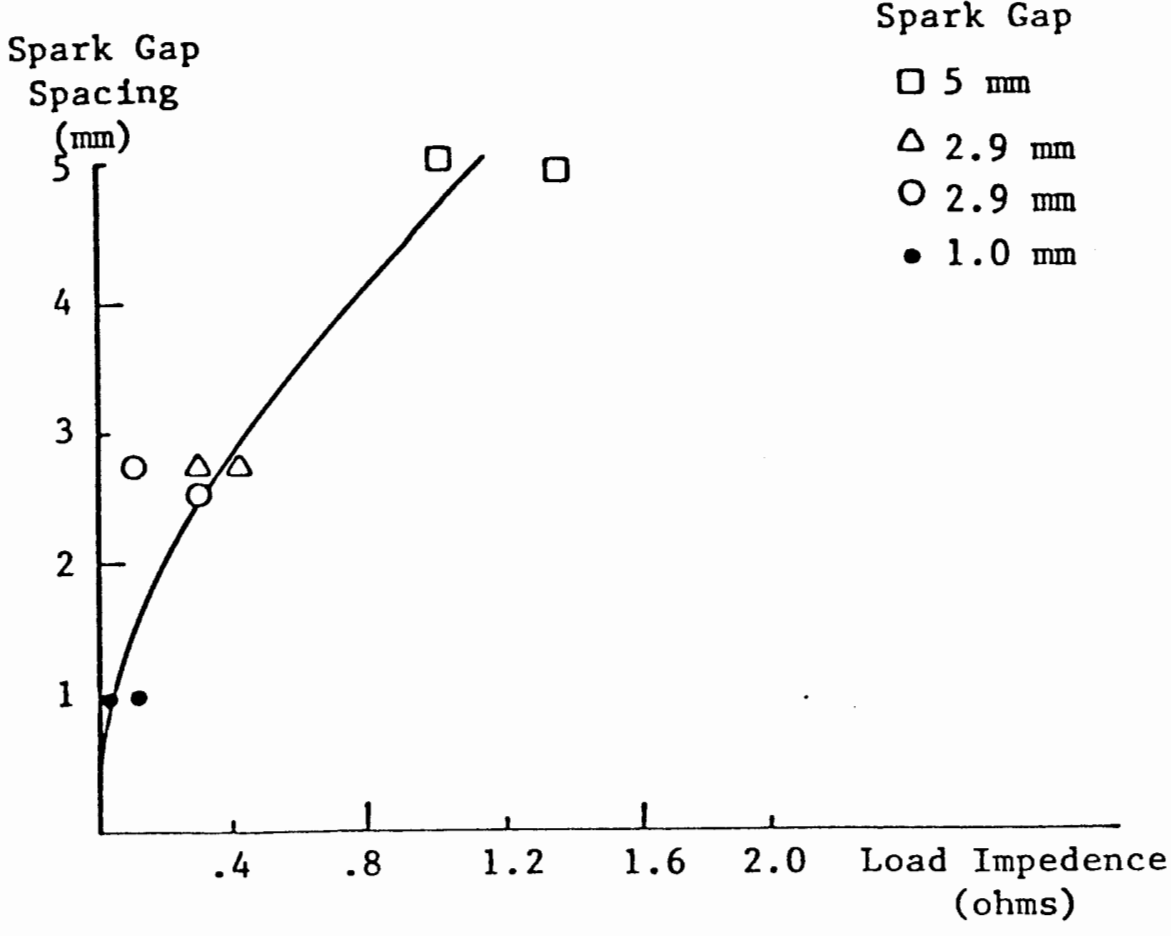

Fig. 6.27. Spark electrode spacing vs. load impedance

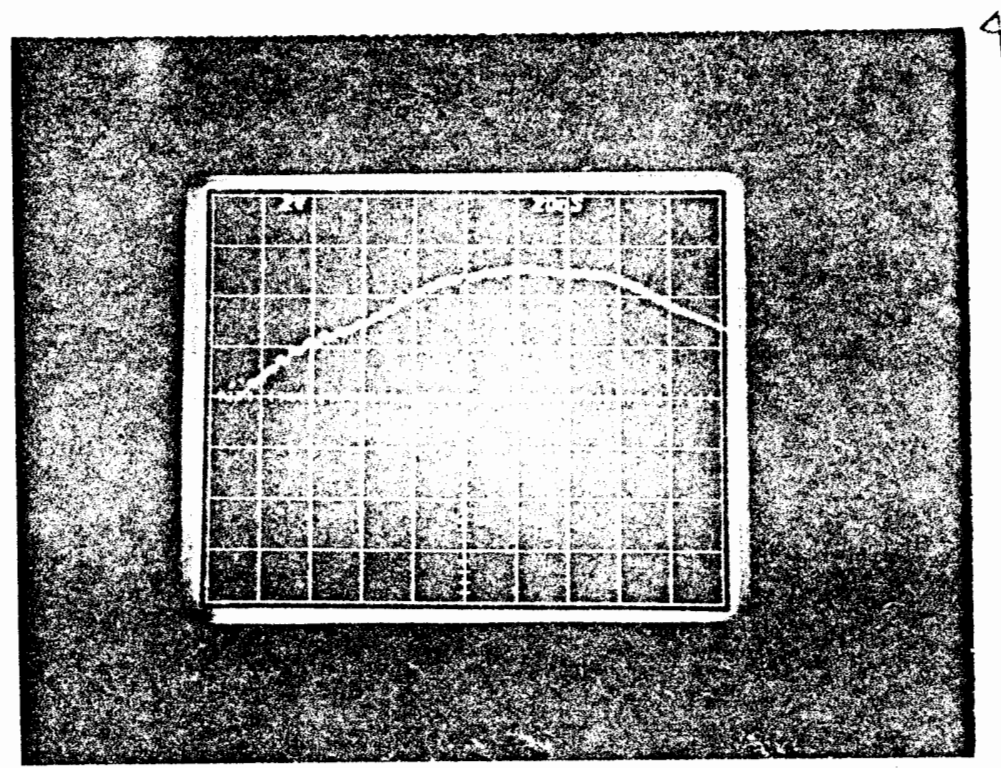

Fig. 6.28. TL current output waveform $\left(Z_{\mathrm{TL}}=.4 \mathrm{ohm}\right)$ 


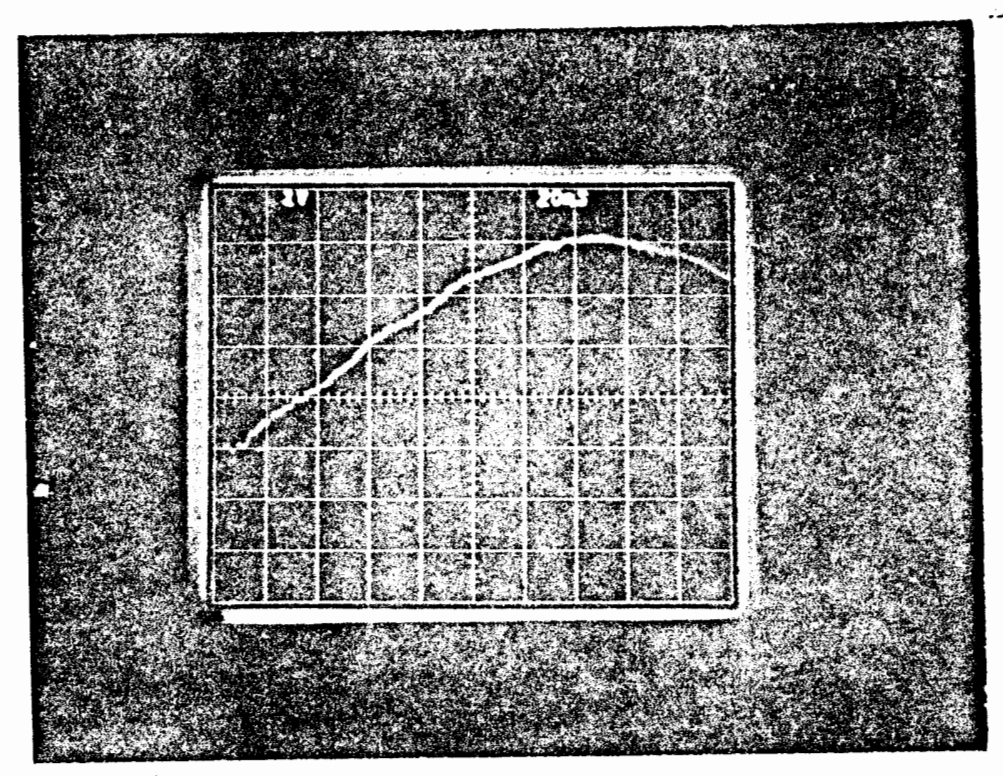

Fig. 6.29. TL current output waveform $\left(\mathrm{Z}_{\mathrm{TL}}=.4 \mathrm{ohm}\right)$

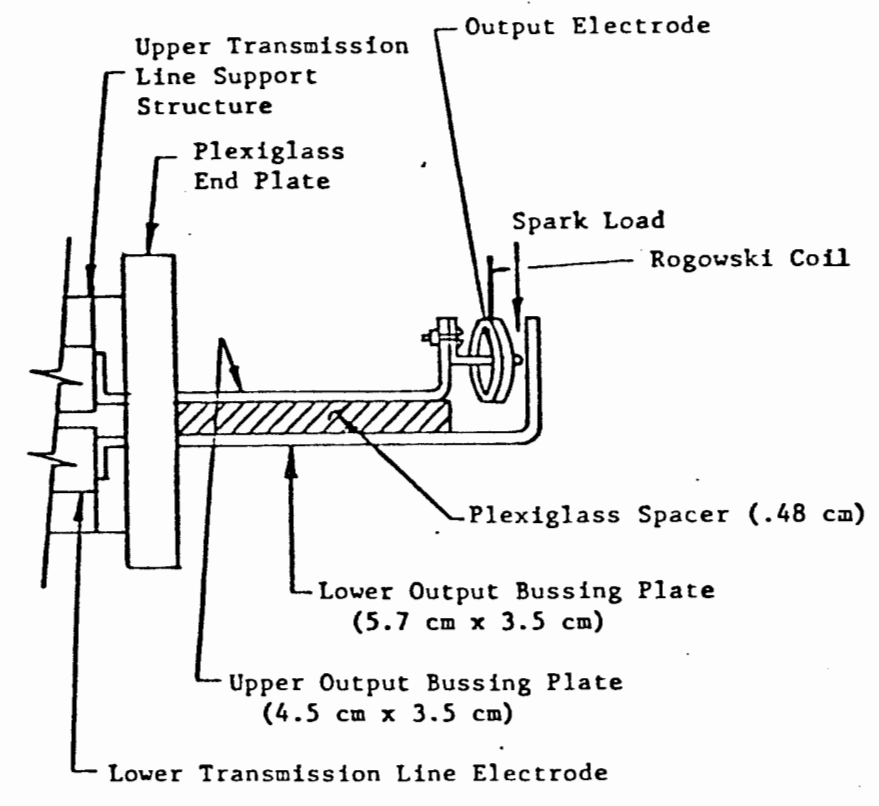

Fig. 6.30. Short busing strip output configuration 
reduce all other contributions to the output inductance to an absolute minimum. Therefore, at the same time that the TL spacing was increased, a new output configuration was also used. This configuration is shown in Figure 6.21. The new configuration utilized the widest possible busing strip from the TL to the spark load, thereby minimizing busing inductance. At the load end it was decided to reduce the arc length to the shortest possible usable value. The arc length in all remaining series of experiments was set at no more than $3.2 \mathrm{~mm}$. The fifth series of experiments (Series E) used this low inductance configuration and a $2.4 \mathrm{~mm}$ TL spacing $\left(\mathrm{Z}_{\mathrm{TL}}=3.58 \Omega\right)$. The risetimes were 16 to $18 \mathrm{nsec}$ long and were characteristic of distributed parameter discharges (See Figures 6.31 through 6.32) Notice how the tops of the discharges are finally leveling out as compared to Figures 6.25 and 6.29. The inclined shape of the top of the pulse is probably the result of the high capacitance Marx-Bank trying to drive the transmission line voltage up during discharge.

The last two sets of experiments, Series $F$ and $G$ were performed after the Marx-Bank had been replaced by the resonant charging network. Micrometers were also added to the upper section of the transmission line which enabled the spacing and hence the TL impedance to be more easily varied to any desired value. The TL impedance was raised to 8.9 ohms and the associated risetimes observed. For the 8.9 ohm spacing, the current risetimes (10-90\%) were no more an 6 nsec.*

*Unadjusted risetimes which did not account for instrumentation errors 


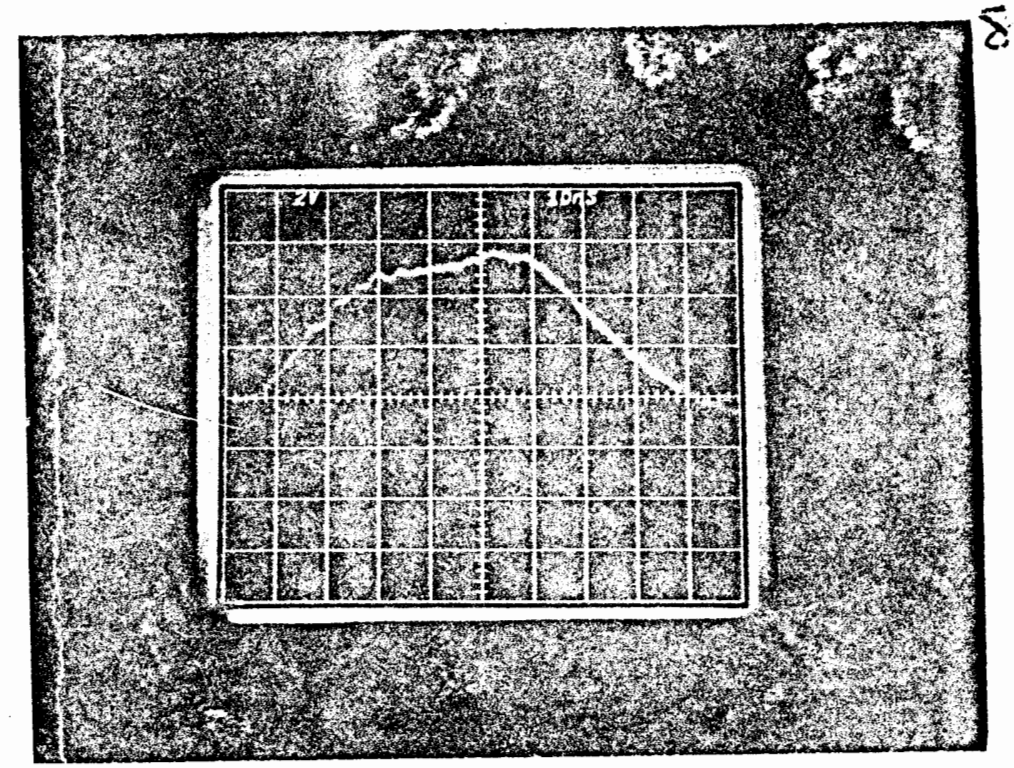

Fig. 6.31. TL current output waveform $\left(Z_{\mathrm{TL}}=3.6 \mathrm{ohm}\right)$

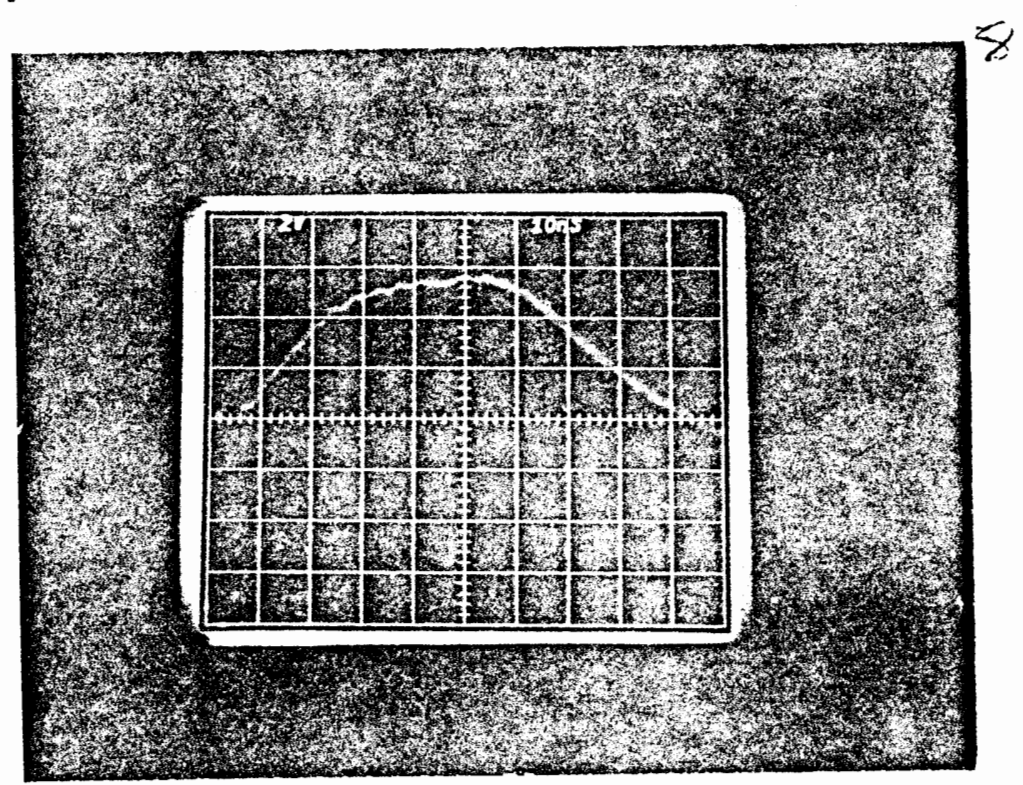

Fig. 6.32. TL current output waveform $\left(Z_{\mathrm{TL}}=3.6 \mathrm{ohm}\right)$ 


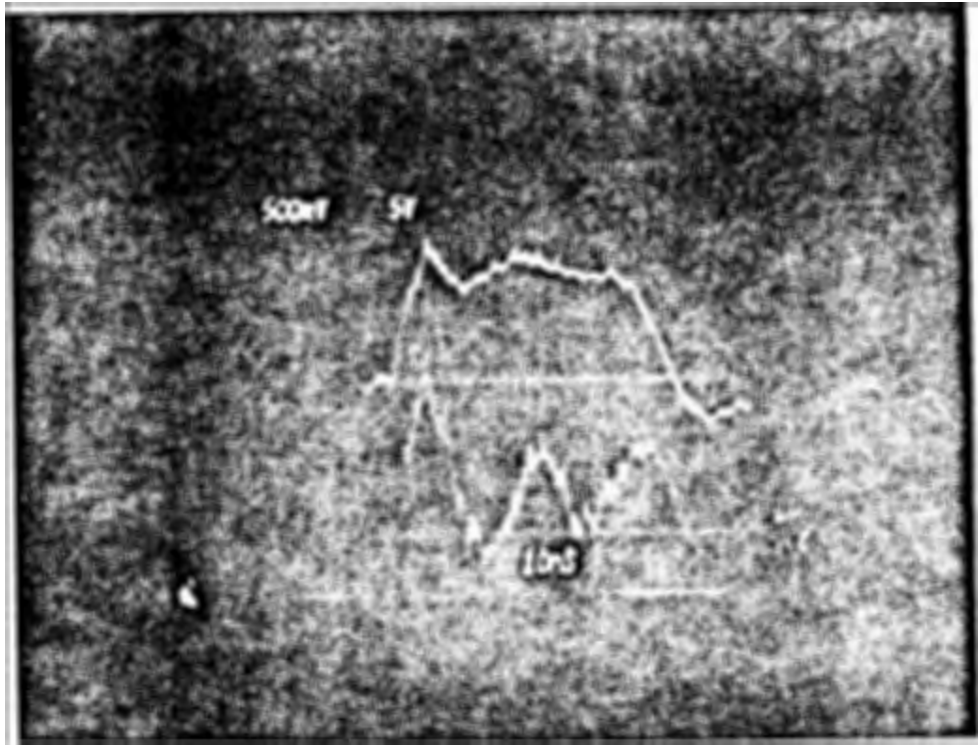

Fig. 6.33. TL current and voltage output waveforms $\left(\mathrm{Z}_{\mathrm{TL}}=8.9 \mathrm{ohm}\right)$

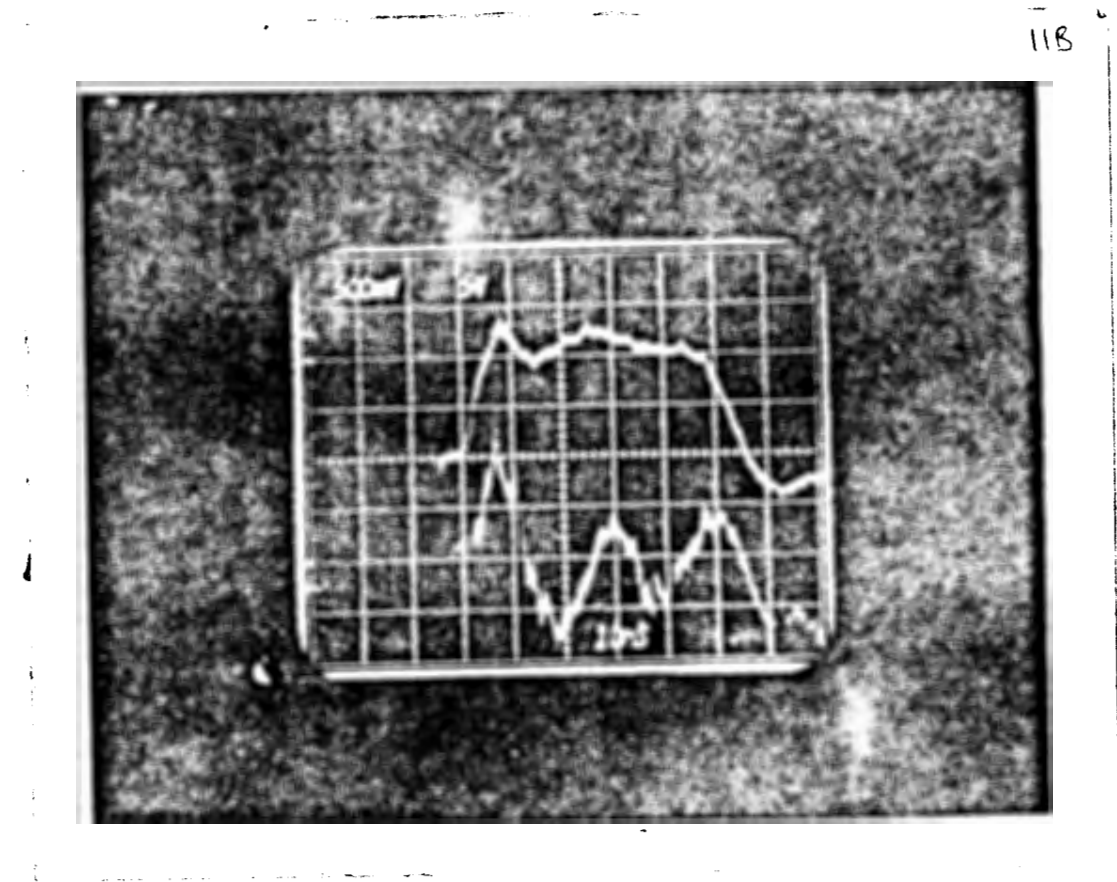

Fig. 6.34. TL current and voltage output waveforms $\left(Z_{\mathrm{TL}}=8.9 \mathrm{ohm}\right)$ 
Figures 6.33 and 6.34 show some of the traces observed on a Tektronix 7844 dual beam oscilloscope. The upper trace is of the current, while the lower is of the voltage. Note that the voltage trace does not show the characteristic rectangular shape that the current trace does. This is in this time frame. Inspection of the traces show that the time period between the first voltage peak after breakdown and the first voltage minimum is about $8-10 \mathrm{nsec}$, substantiating the hypothesis that reflections are taking place. It is also very evident by the traces that the discharge is also highly underdamped. This was a result of trying to minimize the arc inductance and its effect on the risetimes. Unfortunately, this also reduces the load impedance and causes the ringing so noticeable in the traces. Figure 6.35 summarizes the entire series of risetime experiments by plotting risetimes versus the transmission line impedances.

Figure 6.35 shows the TL output current risetimes versus the TL impedence. It should be noted that the fastest risetime pulses were obtained with the highest impedence line tested (i.e., 8.9). The reason for this is that by equation 21 the output current risetime is proportional to the output inductance and inversely proportional to the TL impendence. Therefore, for a given output configuration and hence output inductance, a faster risetime current pulse can be obtained by increasing the TL impedence. Unfortunately, in this specific instance the desired square wave pulse shapes were achieved with a TL impendance much higher than the anticipated laser or flashlamp loads of approximately 1 obm that this system could potentially drive. In order to improve on this mismatch, it would be necessary to reduce the output 
inductance that the TL sees to an absolute minimum. The value of the output inductance can be determined by using equation 21 and the known TL impendance and measured current risetimes. This calculation for tests $D$ through $F$ shows that the output inductance varies from about 40 to 60 nhy. This range of output inductances results from having to vary the output bussing strips separation distances as the TL impendance is changed. The output inductance includes all electrical bussing strips between the TL and spark load and the inductance of the spark itself. It is estimated using equation 12 that the major portion of this inductance (25 to 35 nhy) results from having to introduce the Rogowski coil into the discharge circuit. If another method of current measurement, such as a current shunt, were used, the risetimes obtainable from the lower impendance settings of the TL could be reduced to the 10 to 20 nsec region. 


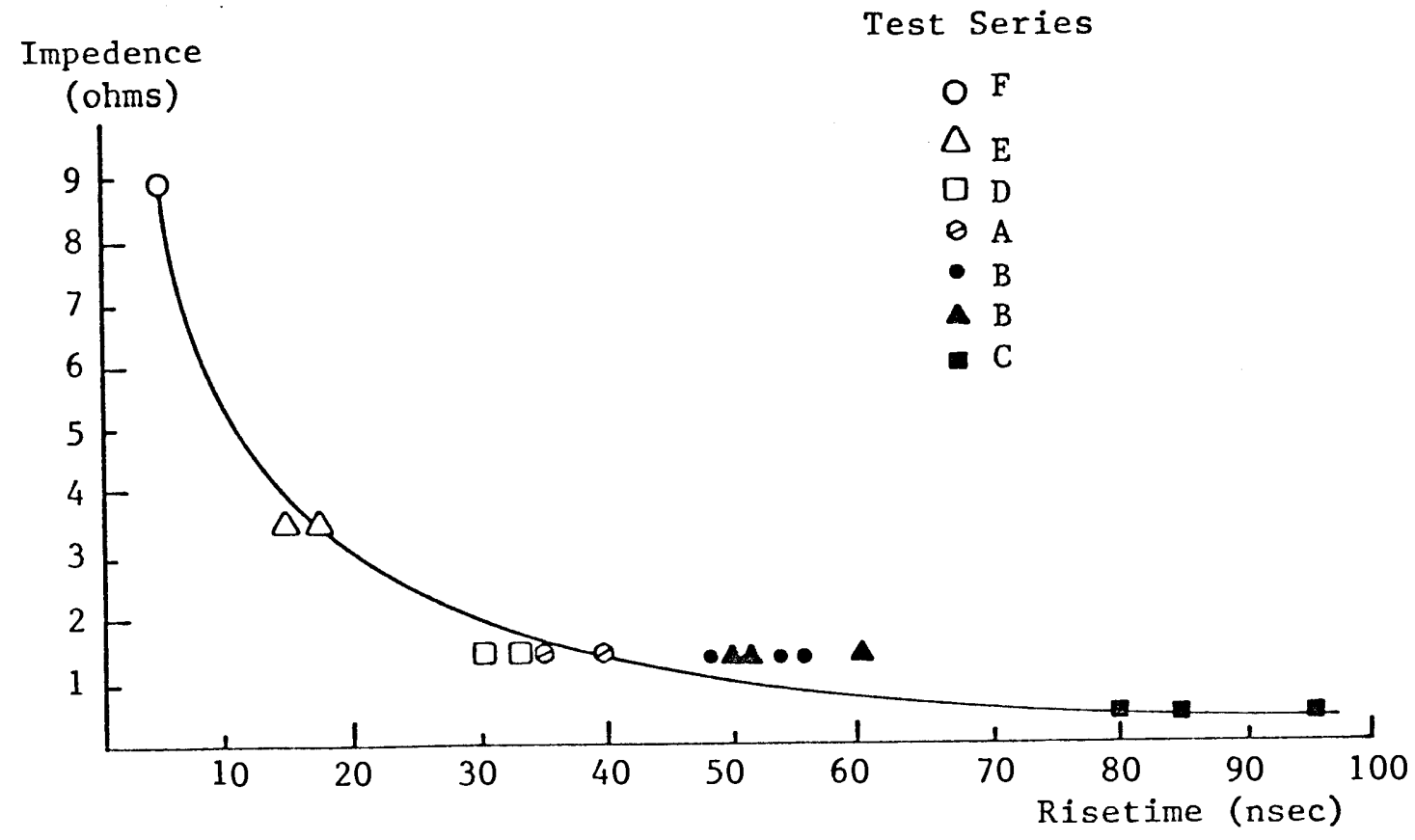

Fig. 6.35. TL output pulse risetime vs TL impedance 


\section{CONCLUSION}

The purpose of this study was the investigation of methods for improving the lifetime and reliability of fast risetime electrical discharge circuits applicable to use in high repetition rate shortwavelength laser schemes. The deficiencies of conventional driving circuits has been shown to be centered around the use of low inductance, high voltage capacitors whose lifetimes are extremely limited under high repetition rate conditions. One approach with potential promise for circumenting this problem is through the use of liquid dielectrics such as water and glycerine whose self-healing properties and stored energy density characteristics appear favorable for use in high repetition rate, fast risetime electrical discharge circuits.

In order to test the effectiveness of such circuits in potential laser applications, a prototype electrical energy storage device has been designed, constructed and laboratory tested. The device was built to operate as a short length hi-voltage transmission line utilizing liquid glycerine as its dielectric. The basic difficulty and expense incurred just building the structural element of the energy storage device suggests that significant reductions in the complexity and cost would have to be achieved for this aspect of the circuit before this type of approach would be acceptable in many applications other than just strictly research ones.

In summary of the set of experiments performed, much has been learned and substantiated concerning the fundamental operating character- 
istics of distributed parameter networks (i.e., transmission line networks) for possible laser excitation. The transition region between distributed parameter and 1 umped parameter discharges (i.e., ordinary capacitor discharges) has been observed and measurements of current and voltage waveforms taken. The predicted inverse relationship between risetime and source impedance has been shown to hold true for varying conditions on the output network. It has also been proven that the current rate of change is definitely proportional to the output inductance which includes both busing and load inductance. During the course of these experiments, it was also found that the need to use a current transformer to measure the current risetimes adversely affected the very parameter that was to be measured. Use of the doughnut shaped Rogowski coil required a significant separation of the current carrying conductors on the load end of the TL. This required separation of the conductors resulted in an additional 25 to 35 nhy of inductance being introduced into the output load, thereby restricting current risetimes attainable at the lower $\mathrm{TL}$ impedence settings of the device. This definitely points out the need to use another method and/or probe for measuring the pulsed current output of the transmission lines. In this context it would be worth considering the use of some sort of current shunt or current viewing resistor which would not have such a deleterious effect on the parameter being measured.

The transmission line tested exhibited the square wave current pulse characteristic of this type of energy storage element. Current risetimes as short as $4 \mathrm{nsec}$ were obtained at the 8.9 ohm 
impedence setting of the TL. Pulse repetition rates of up to $2 \mathrm{khz}$ were achieved. Continuous firing of this system at repetition rates of 1 khz for periods of about five minutes duration (i.e. 300,000 shots) were also achieved without an internal fault occurring in the glycerine dielectric. Longer periods of continuous operation were not limited by the TL, but by the load spark discharge electrodes which became eroded and carbon coated by the high number of successive discharges. This caused the required breakdown voltage for the spark gap to increase and the system at some point would fail to fire reliably. The system had to be shut down and the electrodes cleaned and the spark gap spacing readjusted. Maximum power transfer experiments for optimizing the transfer of energy from the TL to a spark load were not attempted. The major emphasis on testing centered on studying only the TL and its busing network by minimizing the spark load including its inherent inductance. This was done with the knowledge that once the TL and its output busing strips electrical discharge characteristics were known, the optimum load in terms of resistance and inductance could be determined. As originally designed, the system would provide 1 joule of energy per shot from a 1 ohm TL operating into a matched load. The design criterion of only 1 joule per shot was necessitated by desire to also achieve khz repetition rates. The power supplies utilized in this study were a definite limitation to the maximum per pulse energy delivered at high repetition rates in this system.

In conclusion, the use of liquid dielectrics and their self-healing characteristics in reliable, high speed electrical dis- 
charge circuits appear quite favorable as compared to conventional discharge circuitry. While liquids are almost invariably used in e-beam devices, their use in simpler electrical discharge schemes has been highly limited. Further work with liquid dielectrics and their advantages and disadvantages could lead to the construction of devices not only for research type applications, but those pertaining to commercial use also. As an update on the validity of the presented thesis work on liquid dielectrics, the author in the first part of 1983 contacted various capacitor manufacturers to learn what progress had been made in the last several years in the design and construction of hi-repetition rate, low inductance discharge capacitors. It was learned that Maxwell Laboratories had developed, in competition with several other vendors, a long-lived, hi-repetition rate, low-inductance capacitor to be used for a dye laser excitation circuit employed in an uranium isotope separation scheme.

The very objectives and purpose of this work were identical to those which prompted this earlier thesis work. The capacitor was identified as the weak link in attaining high repetition rate, long-lived, short-wavelength laser systems and set in motion a competitive development program among several capacitor manufacturers. The requirements of such an energy storage system are so stringent, as has been pointed out by this thesis work, that two of the four competing manufacturers falled to meet the required goal of extreme longetivity in their capacitor design. The Maxwell design attained lifetimes exceeding $10^{10}$ shots at repetition rates of 550 to 1000 Hertz. This is a vast improvement over earlier capacitors and newer and even more improved versions are anticipated. 
It should be noted that one of the primary features of Maxwell's design was the use of a liquid dielectric as an impregnant in a solid dielectric. The reasons for doing this were exactly the same as those which prompted the author to investigate liquid dielectrics in general for electrical energy storage applications, namely good energy storage capabilities and self-healing characteristics.

In conclusion, while there has appeared commercially an appropriate fast discharge, long-lived capacitor for short wavelength laser applications, the potential for the development of liquid dielectric energy storage element is still considerable. These new capacitor's specifications will define the design requirements for an all liquid dielectric storage element that might be a competitive alternative for some applications. 
It should be emphasized that even though the energy-storage device tested should have considerable promise for use in high repetition rate, fast discharge laser applications, further modification of the prototype device would be necessary before it could be used in laser driving circuits. The modifications are as follows:

1. Design and construct a simple energy storage device utilizing liquid dielectrics. A lightweight, multiple electrode transmission line structure weighing not more than 100 lbs and capable of delivering 10 to 20 joules of energy into a $1 \mathrm{ohm}$ load at repetition rates of $1 \mathrm{khz}$ would illustrate the competitiveness of this type of device as compared to the state-of-the-art solid state capacitors designed for similar application. The device tested in this study had considerably more structural mass than required, and it was limited by the available power supply to delivering only a single joule per shot.

2. Improve the electrical coupling on the input and output sections of the storage device. With proper design the input and output bussing networks self-inductance could be minimized thereby improving on the charging times of the transmission lines and minimizing the output current pulse's risetimes into the load. 
3. Add a flow system and deionization scheme in order to utilize water as the dielectric. A flow system would allow for the cooling and deionization of the water dielectric under extended periods of firing at high repetition rate. This would further test the longevity capabilities of this type of system.

4. Another area which would need improvement is the switch used to pulse charge the transmission line energy storage device. The present switch, an air blown spark gap, is susceptible to carbon buildup after periods of firing at high repetition rates. This leads to unreliable operation and therefore needs improvement. A high power thyrat on or other switch of this type is suggested.

5. A fifth change that is necessary is to do away with the Rogowski coil that was used in the present study in favor of a stripline current monitoring device. The need to insert the doughnut-shaped Rogowski coil in the output loop of the discharge circuit greatly increased the output inductance of the loop, thereby restricting the current risetimes attainable with this particular arrangement.

The above suggested modifications could lead to an improved high repetition rate fast discharge circuit for use in laser excitation schemes. While the present study was aimed at those laser applications requiring direct discharge or flash-lamp excitation, one should not 
overlook other possible applications such as small, high repetition rate electron beam devices. E-beam devices typically use TL energy storage elements in their excitation circuits. E-beam machines have the important attribute that once the beam exits from the device, its pulse energy, width and shape cannot be affected by the load upon which it impinges. This means that a high impedance e-beam machine can efficiently excite a much lower impedance load. It should be noted that e-beam devices have found wide-spread applications in the optics and semi-conductor industry and a high repetition rate machine could increase productivity significantly.

In addition to the proposed modifications and applications, it is important to continue the investigations for other liquid dielectrics which could be used in electrical energy storage applications. An in-depth literature search could reveal other liquids whose electrical properties suggest that they might show improved operational characteristics in fast discharge systems as compared to the liquids studied in this project. Liquids possessing high dielectric strengths or larger permittivities than the liquids studied in this project could increase the stored energy density presently attainable, thereby allowing construction of smaller and lighter structures which would have the same output pulse length and energy delivering capabilities as systems using the more conventional, aforementioned liquid dielectrics. The next step in further determining the capabilities of liquid dielectric transmission lines for hi-power laser applications would be the construction of a new and much larger transmission line 
system. This system should be capable of delivering 20 to 30 joules of energy into a matched 1 ohm load with current risetimes in the 10 to 20 nsec region. A cooling and recirculation system for the liquid dielectric would further demonstrate the advantages of liquid dielectrics as compared to solid dielectrics in hi-power applications. After successful completion of that follow-on phase, the device could be incorporated into a laser. 


\section{APPENDIX 1}

To find the maximum of the power transfer from a resistively charged capacitor to a load, equation (36) is to be first differentiated with respect to the capacitance $C$. The resulting equation is then set to zero and the relationship between $C$ and $R$ and $T$ solved for. Following this procedure we have

$$
\begin{aligned}
& E_{L}=1 / 2 C V^{2}\left(1-e^{-t / R C}\right)^{2} \\
& \left.d_{L} / d C=\frac{d[A C}{d C}\left(1-e^{B / C}\right)^{2}\right]
\end{aligned}
$$

where $A=V^{2} / 2$ and $B=-t / R$

$$
\begin{aligned}
\mathrm{dE}_{L} / \mathrm{dC}= & \frac{\mathrm{d}}{\mathrm{dC}}\left[(\mathrm{AC})\left(1-\mathrm{e}^{\mathrm{B} / \mathrm{C}}+\mathrm{e}^{2 \mathrm{~B} / C}\right)\right] \\
\mathrm{dE}_{\mathrm{L}} / \mathrm{dC} & =(A C)\left[\left(-\mathrm{e}^{\mathrm{B} / C}\right)\left(-\mathrm{B} / \mathrm{C}^{2}\right)+\left(\mathrm{e}^{2 \mathrm{~B} / \mathrm{C}}\right)(-2 \mathrm{~B} / \mathrm{C} 2)\right] \\
& +(\mathrm{A})\left[1-2 \mathrm{e}^{\mathrm{B} / \mathrm{C}}+\mathrm{e}^{2 \mathrm{~B} / \mathrm{C}}\right]
\end{aligned}
$$

Setting $\mathrm{dE}_{\mathrm{L}} / \mathrm{dC}=0$ and solving for $\mathrm{C}$

$$
\begin{aligned}
0 & \left.=(A C)\left[\left(-2 e^{B / C}\right)\right)(-B / C 2)+\left(e^{2 B / C}\right)(-2 B / C 2)\right] \\
& +(A)\left[1-2 e^{B / C}+e^{2 B / C}\right] \\
0 & =1+\left(-2+2^{B / C}\right) e^{B / C}+(1-2 B / C) e 2 B / C
\end{aligned}
$$

Letting $x=e^{B / C}, D=(-2+2 B / C)$ and $H=(1-2 B / C)$, we now have $0=1+\mathrm{Dx}+\mathrm{Hx}^{2}$

This equality may be solved by the use of the binomial theorem. If $x=1$, then since $x=e^{B / C}$, this means that $B=0$. Recalling 
that $B=-t / r$, this requires that either $t=0$ or $R=$. Since neither of these cases is of practical interest, the only remaining solution is $x=1-(2 B / C)$.

$$
\begin{aligned}
& x=[1-2 B / C]^{-1} \\
& e^{B / C}=[1-2 B / C]-1 \text { or }-t / R C=[1+2 t / R C]^{-1}
\end{aligned}
$$

It should be noted that the above expression is transcendental in nature and an exact solution in terms of $C$ does not exist. Nevertheless an approximation can be made by a series expansion of $\mathrm{e}^{\mathrm{B} / \mathrm{C}}$

$$
\mathrm{e}^{B / C}=1+B / C+\frac{(B / C)^{2}}{2 !}+\frac{(B / C)^{3}}{3 !}+\frac{(B / C)^{4}}{4 !}+\ldots
$$

Using only the first four terms in the series

$$
\begin{gathered}
e^{B / C}=1+\frac{B / C}{2 !}+\frac{(B / C)^{2}}{6 !} \\
e^{t / R C}=1+2 t / R C=1+t / R C+\frac{t^{2} / R^{2} C^{2}}{2}+\frac{t^{3 / R^{3} C^{3}}}{6} \\
\frac{t^{3} / R^{3} C^{3}}{6}+\frac{t^{2} / R^{2} C^{2}}{2}-t / R C=0 \\
\frac{t^{2} / R^{2} C^{2}}{6}+\frac{t / R C}{2}-1=0
\end{gathered}
$$

Letting $x=t / R C$

$$
\frac{x^{2}}{6}+\frac{x}{2}-1=0
$$


Again, we can solve this expression by application of the binomial theorem giving

$$
x=-4.37 \text { and } x=1.37
$$

Since a negative value of $x$ can only correspond to a negative time period this case is of no interest. Therefore, the remaining solution can be used to obtain the desired result.

$$
x=t / R C=1.37 \text { or } C=.73 t / R
$$




\section{APPENDIX 2}

The derivation of the expression for the RC decay constant of a TL is based on the product of the equations for the resistance and capacitance of parallel plate capacitor. These equations can be obtained from reference (11) and the derivation performed accordingly. From (11) we have

$$
R=\eta s / A \text { and } c=\epsilon A / s
$$

Taking the product RC we find that the decay constant is independent of geometry and depends only upon the characteristics of the dielectric itself.

$$
R C=(m s / A)(\epsilon A s)=M \in
$$


1. Weast, R. C., 1964, "Persistant Lines of the Elements," Handbook of Chemistry and Physics, 45th Edition, P. E96-E99.

2. Reed, J. Jensen, 1976, "Prospects for Uranium Enrichment," Laser Focus, 12, p. 51-63.

3. Schalow, J. and Townes, C., 1958, "Optical Masers," Physics Review, 112, P. 1940.

4. Schafer, F.P., 1973, Dye Lasers, (New York: Springer-Verlag), p. 54

5. F. T. Arecchi and Schulz-Dubois, E.0., 1972, Laser Handbook (New York: North-Holland Publishing Co.), p. 327-368.

6. Marshall, I.S., 1962, "Strong-Current Pulse (spark) Discharges in Gas, Used in Pulsed Light Sources,", Soviet Physics Uspehi, $\underline{5}$, p. 475 .

7. Reitz, J. R. 1967, Foundations of Electromagnetic Theory, (Reading, Mass.: Addison-Wesley), P. 250.

8. Johnson, G.E., 1971, Basics of Circuit Analysis for Practical Engineering (New York: Barnes and Noble), p. 140.

9. Frunge1, F., 1965, High Speed Pulse Technology, (New York:

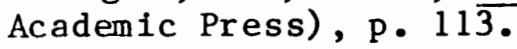

10. Richter, P., Kimel J. D., and Moulton G.C., 1976, "Pulsed Uv Nitrogen laser: Dynamical Behavior," Applied Optics, 15, p. 756-760.

11. Sorokin, P.P., et al, 1968, "Flashlamp-Pumped Organic-Dye Lasers," Journal of Chemical Physics, 48, p. 4726-4741.

12. Aristov, A.V. et al, 1969, "Influence of Certain Quenchers of Fluorescence on the Threshold of the Laser Effect in Organic Phosphors," Optical Spectroscopy, 27, p. 548.

13. F. Emmett, et a1., 1977, "ArF and keF lasers," Applied Physics letters, $\underline{36}, \mathrm{p} .418$.

14. Booker, H.G., 1959, An Approach to Electrical Science, (New York: Mc Graw-Hi 11) , p , 548 .

15. Hoffman, P. and Ferrante, J. "Energy Storage Capacitors of High Energy Density," IEEE Transactions on Electrical Insulation, 17, p. 235-239. 
16. Hayworth, B.R., 1968, "The Behavior of Polyester Film Energy Storage Capacitors," IEEE Transactions on Electrical Insulation, $\underline{3}$, p. 47

17. Capacitor Specialists, Inc., P.0. Box 2052, Escondido, Calif., CSI Data Sheet No. 0674-WM on HI-Voltage Energy Storage Capacitors.

18. J. C. Martin, 1970, "Nanosecond Pulse Techniques," Atomic Weapons Research Establishments Note No. SSWA/JCM/704/49 Aldemastion, England.

19. Olson, N.T., 1976, "The Power Behind the Pulse," Optical Spectra, 10, p. 42-46.

20. Weszak, L., 1969 "Power-Supply Design for Pulsed Lasers," Microwaves, 21 , p. 38 .

21. Glascoe, and Lebacqz, 1965, Pulse Generators, (New York: Dover)

22. D. G. Sutton, et al., 1976, "Fast-discharge-initiated $\mathrm{KrF}$ laser, "Applied Physics letters, 28, p. 522.

23. Von Hipple, E. 1954, Dielectric Materials and Applications (New York: Wiley), p. 361-368.

24. V. Valencia, et al., 1983 "High repition rate, long life capacitors developed for laser isotope separation modulators," Unpublished paper from Maxwel1 Laboratories, Inc., San Diego, California 